\title{
CONFORMATION AND CHIRALITY IN LIQUID CRYSTALS
}

\author{
A dissertation submitted to \\ Kent State University in partial \\ fulfillment of the requirements for the \\ degree of Doctor of Philosophy
}

by

Lei Zhao

August, 2018 
Dissertation written by

Lei Zhao

B.S., Nanjing University, China, 2008

Ph.D., Kent State University, USA 2018

Approved by

Dr John L. West , Chair, Doctoral Dissertation Committee

Dr Philip Bos , Members, Doctoral Dissertation Committee

Dr Deng-Ke Yang

Dr Elizabeth Mann

Dr Michael Fisch

Accepted by

Dr Antal Jakli , Chair, Department of Chemical Physics Interdisciplinary Program

Dr James L. Blank , Dean, College of Arts and Science 


\section{TABLE OF CONTENTS}

Table of contents $\ldots \ldots \ldots \ldots \ldots \ldots \ldots \ldots$ iii

List of figures $\ldots \ldots \ldots \ldots \ldots \ldots \ldots \ldots$ vi

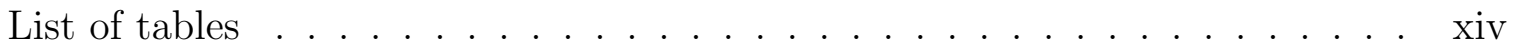

Acknowledgements ..................... . . xv

Dedication . . . . . . . . . . . . . . . . xvi

1 Introduction ............................. 1

1.1 Introduction of chirality $\ldots \ldots \ldots \ldots \ldots \ldots$

1.1 .1 Chirality . . . . . . . . . . . . . . . . . 1

1.1.2 Chirality in Daily Life $\ldots \ldots \ldots \ldots \ldots$

1.1.3 Chirality in chemistry and materials science . . . . . . 10

1.2 Thesis Overview and Organization $\ldots \ldots \ldots \ldots$

2 Cholesteric Liquid Crystals and Chiral Dopants . . . . . . . . . . . . 22

2.1 Liquid Crystals and Liquid Crystalline Phases . . . . . . . . . . 22

2.2 Chiral Liquid Crystals and Helical Twisting Power (HTP) _ . . . . 29 
2.3 Helical Twisting Power . . . . . . . . . . . . . . . . . . . 34

$2.4 \quad \theta$-cell Measuring Helical Twisting Power . . . . . . . . . . . . . . . . 37

2.5 Other methods Measuring Helical Twisting Power . . . . . . . . . . . 48

2.5.1 Reflective spectroscopic Method . . . . . . . . . . . . . . 48

2.5.2 Wedge Cell Method . . . . . . . . . . . . . . . . . . . . 48

2.5.3 Bragg Scattering Method ............... 54

2.5.4 TN Cell Method .................. 54

2.6 Conclusion . . . . . . . . . . . . . . . . . . 59

3 Conformation and Chirality in Liquid Crystals . . . . . . . . . . . . . 60

3.1 Introducing a Helical Structure into an Achiral Host . . . . . . . . . . 60

3.2 Chiral Conformer . . . . . . . . . . . . . . . . . 65

3.3 Conformation and Chirality . . . . . . . . . . . . 76

3.4 Conclusion . . . . . . . . . . . . . . . . 83

4 Mechanism of Temperature Dependence of Pitch in High Helical Twisting

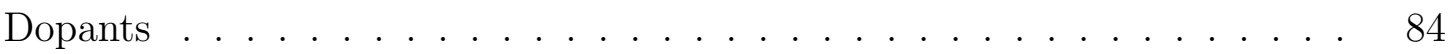

4.1 Introduction . . . . . . . . . . . . . . . . . 85

4.2 Experimental Details . . . . . . . . . . . . . . . . . . 87

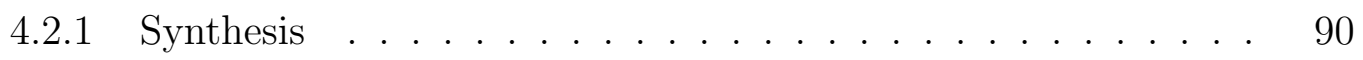

$4.2 .2 \quad \mathrm{UV}-\mathrm{Vis}$ Spectroscopy . . . . . . . . . . . . . . . . 90 
4.3 Results and Discussions . . . . . . . . . . . . . . . . . . . . . . . . . 92

4.3.1 NMR Spectroscopy . . . . . . . . . . . . . . . 110

4.4 Summary and Conclusions . . . . . . . . . . . . . . . . 124

5 Interaction between Chiral Dopant and Liquid Crystal . . . . . . . . . . 126

5.1 Introduction . . . . . . . . . . . . . . 126

5.2 Experiment and Discussion . . . . . . . . . . . . . . . . 129

5.3 Conclusion .......................... 143

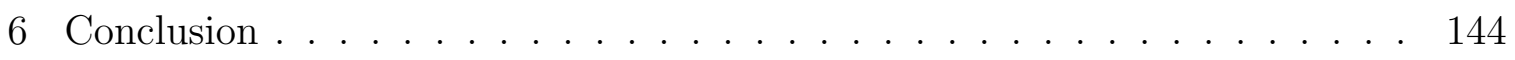

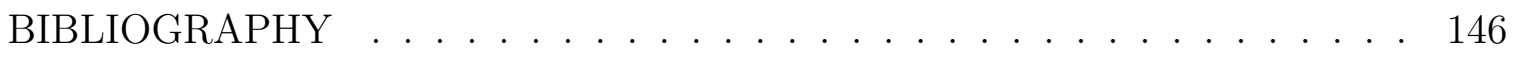

Appendix: Isosbestic Point . . . . . . . . . . . . . . 155 


\section{LIST OF FIGURES}

1 Drawing Hands by M.C. Escher . . . . . . . . . . . . . . . . 3

2 Lefty's The Left Hand Store in San Francisco Pier 39 . . . . . . . . . 6

3 US Postal Servic Truck . . . . . . . . . . . . . . . . . . . 9

4 The (2R,3R)- and (2S,3S)- tartrates were isometric, non-superposable mirror images of each other. . . . . . . . . . . . . . . . . 12

$5 \quad$ R-carvone smells like caraway, and S-carvone smells like spearmint . . 13

$6 \quad$ Friedrich Reinitzer $(1857-1927)$. . . . . . . . . . . . 15

7 Molecular structure of (S)-4'-(2-methylbutyl)[1,1'-biphenyl]-4-carbonitrile

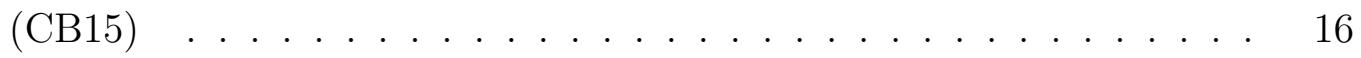

8 CB15, a cholesteric liquid crystal used in the manufacturing of liquid crystal displays, at 100-times magnification. Image by Dr. Christian Bohley, Otto-von-Guericke-University of Magdeburg. . . . . . . . . . 17

9 Boogie Board Jot 8.5 eWriter. . . . . . . . . . . . . . . . . . . 19

10 Pierre-Gilles de Gennes (1932-2007) . . . . . . . . . . . . . 23

11 Phase Diagram for a typical soap-water system . . . . . . . . . 25 
12 Schematic representations of (a) a nematic LC phase, (b) smectic LC phases oriented along (left) and away (right) from the normal of the layer, and (c) a chiral LC phase. . . . . . . . . . . . . 26

13 Cholesteric liquid crystal phase. . . . . . . . . . . . . . . 28

14 Top: Blue Phase double twist structure; Bottom: Nematic Phase (N),

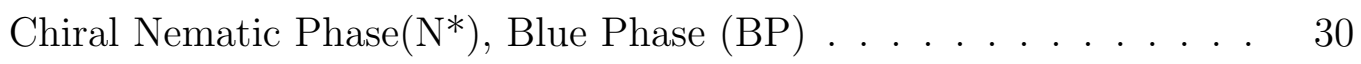

15 Sketch of layered organization of smectic $C^{*}$ phase . . . . . . . . . . 32

16 Left:The rubbing of substrates:front substrate with linear alignment, rear substrate with circular alignment. Right: The orientation of LC molecules, a view from the linear to the circular rubbing . . . . . . . 38

17 Photograph of the cell with inhomoge- neous thickness in crossed polarizers. The cells filled with composi- tion 5CB and S-811: $c=3.7 \times 10^{-3}$

. The numbers indicate the local thickness of the cell. . . . . . . . .

18 Photograph of 5CB doped with CD6 at different concentration in $\theta$-cells. 42

19 The same cells heated up to isotropic phase, then slowly cool down to room temperature ...................... 43

20 Twist angle of disclination line in $\theta$-cells vs concentration of the chiral dopant in liquid crystal host. . . . . . . . . . . . . 46

$21 n^{2}$ as a function of wavelength for two normal modes in bulk CLCs. The dotted line in mode 2 is negative. . . . . . . . . . . . . . 49 
22 Setup of Grandjean-Cano Method.The incident light is monochromatic. 50

23 Left: liquid crystal disclination line observed through optical microscopy; right: distance to the wedge tip is linear with the number of disclination lines . . . . . . . . . . . . . . . . . . . . 52

24 Disclination rings in advanced Crandjean-Dano method, using lens as one substrate, $2 \% \mathrm{CD} 6+98 \% \mathrm{E} 7$ between two crossed polarizers. Radius of Curvature $R=51.680 \mathrm{~mm}$, Newport.com . . . . . . . . . . . 53

25 Theoretical reflection spectra for (a) incident right circular polarization and (b) unpolarized light at five angles of incidence. . . . . . . . . . 55

26 A twisted nematic cell showing degenerate left- and right-hand domains separated by a disclination line. . . . . . . . . . . . . 56

27 A twisted nematic cell showing degenerate left- and right-hand domains separated by a disclination line. . . . . . . . . . . . . 58

28 Schematic illustration of the BPCA-C6-P dimmer orientation in the helix ................................. 62 
29 Propeller-patterned chiral N POM textures of BPCA-C7-PmOH in a bare glass cell: POM textures a) without and b) with a tint-retardation plate $(530 \mathrm{~nm})$ between the analyzer and the sample at $216 \mathrm{C}$. c) Computer-simulated molecular-packing model in a right-handed propellerpatterned $\mathrm{N}$ droplet. Here, $\mathrm{n}$ is the LC molecular director with the twist angle $(\theta)$ from the cylinder axis $(z), r(\phi)$ is the projection of $n$ onto the $r_{x} r_{y}$ plane, and $\phi$ is the azimuthal twist angle. Calculated optical textures based on the molecular arrangement in (c) under POM d) without and e) with a tint-retardation plate between the analyzer and the sample.......................... 64

30 Ethane Barrier: Energy as a function of rotation around C-C bound . 66

31 Energy as a function of rotation around center C-C bound in butane 68

32 Energy as a function of rotation around center C-C bound in butane 69

33 Energy as a function of rotation around center C-C bound in biphenyl 75

34 Dihedral angle between phenyl rings vs number of ring members . . . 77

35 Boat (top) and chair (bottom) conformers for chclohexane . . . . . 79

36 Boat-like (top) and chair-like(bottom) conformers for isosorbide core . 81

37 CD6 with boat-like core(top) and chair-like core(bottom) . . . . . . 82

38 Molecular Structure of the isosorbide benzoate ester studied . . . . . 88

39 Method of Synthesis, using CD9 as an example . . . . . . . . . . . 91 
40 UV-Vis absorption spectroscopy of CD6 in hexanes temperature de-

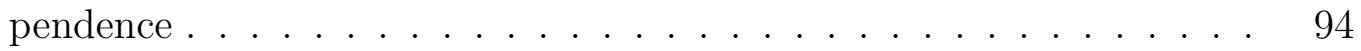

41 Differential absorption relative to absorbance at 0C for CD6 in Octane 95

42 UV-Vis absorption spectroscopy of CD9 in hexanes temperature dependence .......................... 96

43 Relative UV-Vis absorption spectroscopy of CD9 in hexanes temperature dependence, using $25^{\circ} \mathrm{C}$ as baseline . . . . . . . . . . . . . . 97

44 UV absorption spectrum of CD6 dissolved in octane as a function of

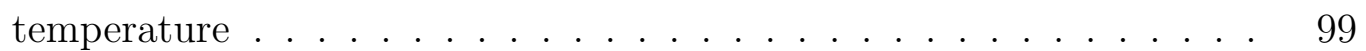

45 Differential absorption relative to absorbance at 0C for CD6 in Octane 100

46 Isosbestic point exists in CD6 regardless of the solvent materials used. 101

47 Differential absorption spectra of upper left: CD3, upper right: CD4, lower left, CD5 and lower right CD7 . . . . . . . . . . . . 103

48 Differential absorption spectra as a function of temperature for CD 9104

49 CD9: molecular model for the structure of the boat (left) and chair core conformation (right) . . . . . . . . . . . . . . 105

50 HTP temperature dependence of CD6 and CD9 . . . . . . . . 106

51 Predominant molecular conformations of CD6 . . . . . . . . . 107

52 Relative distribution between 2 conformers based on temperature and the difference in their free energy . . . . . . . . . . . . . . . . 109 
53 Chair and boat structure of CD3 with proton marks . . . . . . . . . . 112

54 NMR spectra vs temperature change of protons on the isosorbide core $\operatorname{rings} \ldots \ldots \ldots \ldots \ldots \ldots \ldots$

55 NMR spectra of CD3 at room temperature . . . . . . . . . . . 115

56 NMR spectra of of proton 1a, 1b, 6a, 6b at room temperature . . . 116

57 NMR spectra of of proton $1 \mathrm{a}, 1 \mathrm{~b}, 6 \mathrm{a}, 6 \mathrm{~b}$ at $-40^{\circ} \mathrm{C} \ldots \ldots . \ldots 117$

58 NMR spectra of of proton 1a, 1b, 6a, 6b by temperature change . . . 118

59 NMR spectra of of proton 2, 5, 4, 3 by temperature change . . . . . 119

60 NMR signal verses temperature of protons $1 \mathrm{a}, 1 \mathrm{~b}, 6 \mathrm{a}, 6 \mathrm{~b} \ldots \ldots$

61 NMR signal verses temperature of protons 2 and $5 . \ldots . . . . . .121$

62 NMR signal change by temperature of proton 3 and 4 . . . . . . . . 122

63 Coupling constant change between protons vs temperature . . . . . . 123

64 Two phenyl rings in the biphenyl twist conformations in either direction, and the two equal energy states switches rapidly at room temperature. Adding a chiral dopant could make one conformation lower in energy. . . . . . . . . . . . . . . . . . . . . . . . 128 
65 Left: Pure E7, Pure CD6 in Octane UV-Vis absorption spectra, $c(E 7)=$ $3.28 \times 10^{-5}(w t),. c(C D 6)=1.8 \times 10^{-5}(w t)$. Right: Calculated $5 \%$ CD6 in E7 mixture spectrum matches real measurement at different temperature, which indicates no intermolecular interaction in solvent environment. ..................... 130

66 a) liquid crystal mixture coated on PI2555 pre-coated quartz substrate. b) vacuum assemble two identical plates into a cell. c)UV Vis spectroscopy optical path, two PI 2555 pre-coated plates as reference. . . 133

67 a) liquid crystal mixture coated on PI2555 pre-coated quartz substrate. b) vacuum assembly of two identical plates into a cell. c)UV-Vis spectroscopy optical path, two PI 2555 pre-coated plates are used as refer-

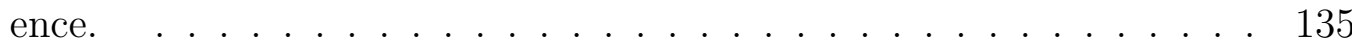

68 a) thin film UV Vis spectra of $3 \%$ of CD3 dissolved in E7 b)thin film UV Vis spectra of $3 \%$ of CD3 dissolved in E7 . . . . . . . . . . 138

$69 \chi_{2}$ vs concentration of chiral dopant . . . . . . . . . . . . . 139

70 Left: $5 \mathrm{CB}$ thin film cell UV-Vis absorption spectra at different temperature. Right: E7 thin film cell UV-Vis absorption spectra at different

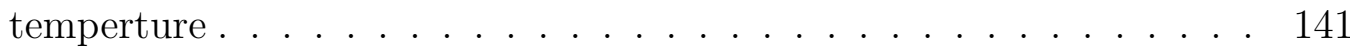


71 Left: 5CB with 2\% CD6 thin film cell UV-Vis absorption spectra at different temperature. Right: E7 with 3\%CD6 thin film cell UV-Vis absorption spectra at different temperture . . . . . . . . . . . . 142

72 Time dependent UV-VIS spectrum of (dichloromethyl)benzene in water at $25^{\circ} \mathrm{C} \ldots \ldots \ldots \ldots \ldots \ldots \ldots$ 


\section{LIST OF TABLES}

1 List of recent U.S. Presidents by handness from Wikipedia . . . . . 8

2 Sample number and the concentration of R811(chiral dopant) . . . . 45

3 Table of energy vs dihedal angle $-1 \ldots \ldots$. . . . . . . . . . . . 72

4 Table of energy vs dihedal angle $-2 \ldots \ldots$. . . . . . . . . . . . 73

5 Table of ring non-planarity modeling . . . . . . . . . . . . . 74

$6 \quad$ The comparison of noise-robustness by four types of basis functions. . 89 


\section{Acknowledgements}

I thank my advisor Dr John L. West for his great support and advises all the time. I also would like to thank my committee: Dr Philip Bos, Dr Deng-Ke Yang, Dr Elizabeth Mann, Dr. Michael Fisch, thank Chair of CPIP: Dr Antal Jakli; and thank Senior Secretary of CPIP: Mary Lyn Bergstrom. Without them defending this dissertation would not have been possible.

Thank Donald Diehl for the synthesis and purification of the series of isosorbide benzoate esters used in this study. We also thank Philip Westerman for his collaboration in obtaining the NMR measurements. I also thank Dr Dawei Lee, Dr Yuri Reznikov, Dr Erica Montbach, Dr Asad Khan, Dr J. William Doane for insightful discussions.

I would also like to express my gratitude to all the LCI members, all my friends and family

This work was funded in part by Kent Displays through grants from the Ohio Third Frontier, ERCP 09-077 and AMP 1078 
This dissertation is dedicated to my parents Sun, Zhen, and Zhao, Jiankui, for their endless love, support and encouragement. 


\title{
CHAPTER 1
}

\author{
Introduction
}

\subsection{Introduction of chirality}

\subsubsection{Chirality}

Chirality, a mysterious property of nature, has interested not only scientists, but also artists and philosophers for a long time. The word chiral, came from the Greek word $\chi \epsilon \iota \rho$ (kheir), "hand", and means handness. It was firstly used by Lord Kelvin [1], and like the relationship of the left hand to the right hand, chirality describes things that cannot superimposed on their mirror images.

M.C. Escher is an artist who had an extreme interest in the chiral world. Many of 
his works reveal this interest. For example, his lithograph Drawing Hands is one of the most famous work that brings chirality and enantiomers into artwork. See Figure 1.

As early as 1783, Kant first discussed the asymmetric issue when looking into mirror. He found the mirror images somehow look the same as the original object, but not exactly. He found that left and right are inverted in the world inside the mirror. Immanuel Kant asked this question in his book Prolegomena [2]:

"What can be more similar in every respect and in every part more alike to my hand and to my ear, than their images in a mirror? And yet I cannot put such a hand as is seen in the glass in the place of its archetype; for if this is a right hand, that in the glass is a left one, and the image or reflection of the right ear is a left one which never can serve as a substitute for the other." And he said, " There are in this case no internal differences which our understanding could determine by thinking alone. Yet the differences are internal as the senses teach, for, notwithstanding their complete equality and similarity, the left hand cannot be enclosed in the same bounds as the right one (they are not congruent); the glove of one hand cannot be used for the other." 


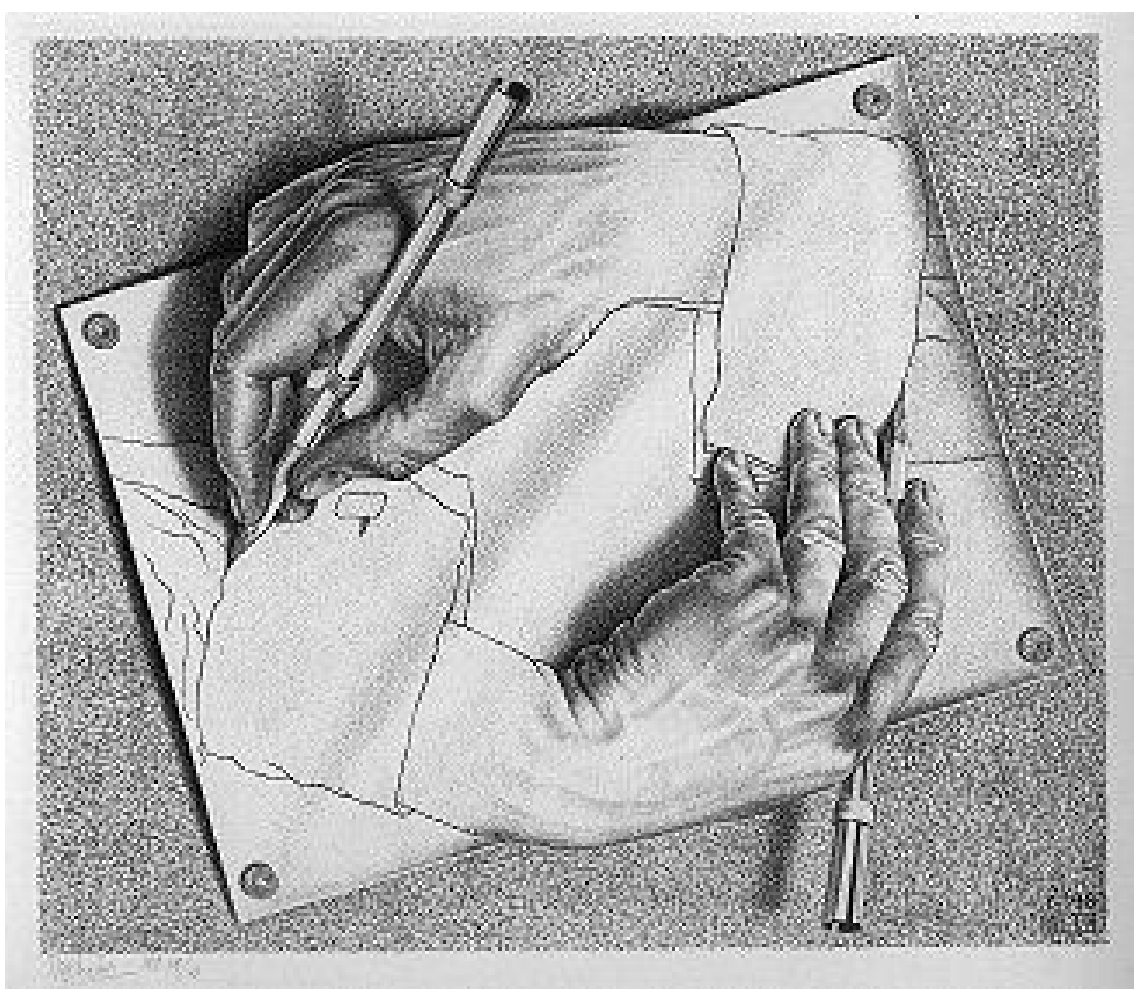

Figure 1: Drawing Hands by M.C. Escher 
Mathematicians followed the philosophers' curiosity. August Ferdinand Möbius is one of the mathematicians who rigorously studied this phenomenon. His theory indicated that, in order to convert one object from one enantiomer to the other, it has to rotate into one order higher dimension. [3] For example, to be superimpose-able on its mirror image, one 2D chiral object has to rotate into 3-dimensional space; enantiomorphous image obtained by reflection in n-dimensional space are completely congruent by rotation in a $(n+1)$ th dimension. The novel Flatland [4] also adopted this theory, and generated an interesting story based on it. In the book, a scalene triangle and an oriented circle wants to convert into its enantiomer. In the 2-dimensional world, they can never make the conversion, so they have to move into the 3 dimensional world to execute the change. The story is the same in our 3-dimensional world. So, if Kant could flip his left hand and ear in a 4-dimensional world, he could convert them to be identical to his right hand and right ear.

While we were getting closer to a fundamental understanding of chiral phenomena, the American mathematician, Martin Gardner, raised a question. Gardner claims that the left- or right- handness convention would arise if we enter into communication with life on another planet. Two intelligent civilizations cannot reach agreement on handness description on an asymmetric object without a prior understanding. They can perform any experiment they want, using for instance, magnets, electrical currents, 
light polarization, gyroscopes etc. But none of them can solve the problem, unless they are able to introduce the concept of chirality in the of universe. For example, the beta decay in which parity is not conserved can be used to distinguish humans leftright spatial relations. Since this problem is first raised during the Project Ozma [5], it is called Ozma Problem.

\subsubsection{Chirality in Daily Life}

Although chirality at first seems to be a novel condition, in fact, chirality is quite common, and exists everywhere in our daily life. As we all known, most of the scissors, or flaps for zippers, are designed to fit a right hand. So left handed people a compelled to use them with an uncomfortable right hand, or uncomfortably use their left hand to use the right-hand oriented tools. Businessman understand this opportunity, and sell tools designed for the left hand. Figure 2 shows a store called Lefty's. It is located in Pier 39, San Francisco. This store specializes in selling tools for left-handed people. In their website, they mentioned that left-handed scissors are their best seller. 


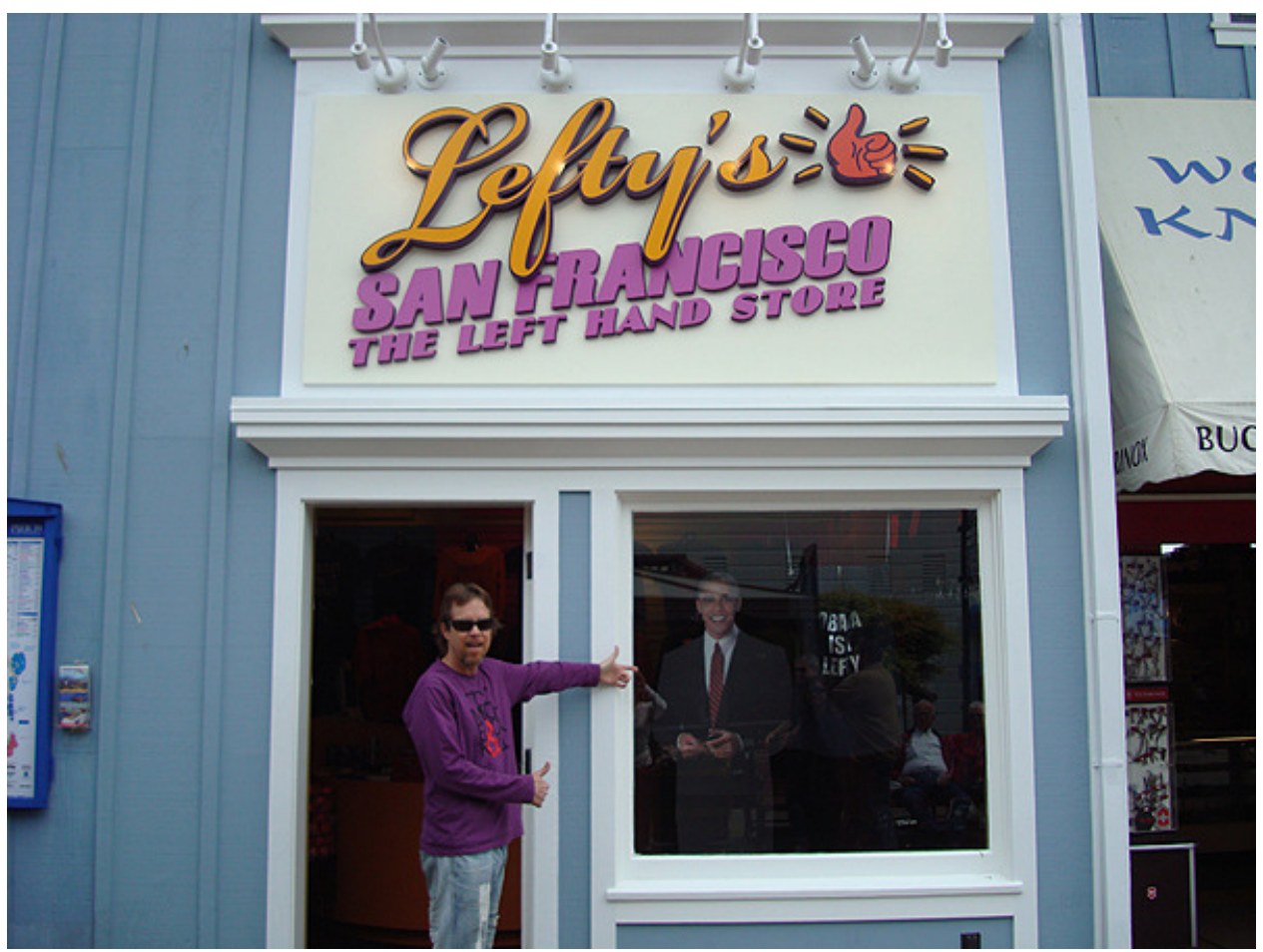

Figure 2: Lefty's The Left Hand Store in San Francisco Pier 39 
In Lefty's photo, we can see President Obama's photo is set by the window. People actually found that 3 out of the 4 most recent presidents are left-handed. [6], see Table1. Some sociologist, who specialize in studying left-handed people, even claim that left-handed people "have a wider scope of thinking", and point to the disproportionately high number of Nobel Prize winners, writers, and painters who are left-handed.

Of course, tools designed for hands are more easily related to the handness and chirality. Actually, handness widely exists in our world well beyond hand-held tools. Every morning, when we go to work, we need to put on cloths, if we look carefully, the suit is actually chiral. The buttons are usually located on one side, and button holes on the opposite. Then we get into cars, and find cars are also chiral. The driver's seat with the steering wheel is always on one side of the car in one country, excepting special cases, such as a postman's vehicle. (See Figure 3)The chirality of the car is usually based on the chirality of the road. The driver's seat is located on the opposite side of the most used passenger doors. This is designed for more conveniently loading and unloading passengers. That is why in US and Mainland China, the steering wheel is on the left side, and when driving in Japan or UK, it is on the right side of the car.

In the modern world, it is more like a global convention that suits have buttons 
Table 1: List of recent U.S. Presidents by handness from Wikipedia

\begin{tabular}{|l|l|l|l|}
\hline \multicolumn{1}{|c|}{ President } & \multicolumn{1}{|c|}{ Party } & \multicolumn{1}{|c|}{ Term } & \multicolumn{1}{c|}{ Handedness } \\
\hline Calvin Coolidge & Republican & $1923-1929$ & Right-handed \\
\hline Herbert Hoover & Republican & $1929-1933$ & Left-handed[dubious - discuss] \\
\hline Franklin D. Roosevelt & Democratic & $1933-1945$ & Right-handed \\
\hline Harry S. Truman & Democratic & $1945-1953$ & Left-handed [5] \\
\hline Dwight D. Eisenhower & Republican & $1953-1961$ & Right-handed \\
\hline John F. Kennedy & Democratic & $1961-1963$ & Right-handed \\
\hline Lyndon B. Johnson & Democratic & $1963-1969$ & Right-handed \\
\hline Richard Nixon & Republican & $1969-1974$ & Right-handed \\
\hline Gerald Ford & Republican & $1974-1977$ & Left-handed \\
\hline Jimmy Carter & Democratic & $1977-1981$ & Right-handed \\
\hline Ronald Reagan & Republican & $1981-1989$ & Ambidextrous \\
\hline George H. W. Bush & Republican & $1989-1993$ & Left-handed \\
\hline Bill Clinton & Democratic & $1993-2001$ & Left-handed \\
\hline George W. Bush & Republican & $2001-2009$ & Right-handed \\
\hline Barack Obama & Democratic & $2009-p r e s e n t$ & Left-handed \\
\hline & & & \\
\hline
\end{tabular}



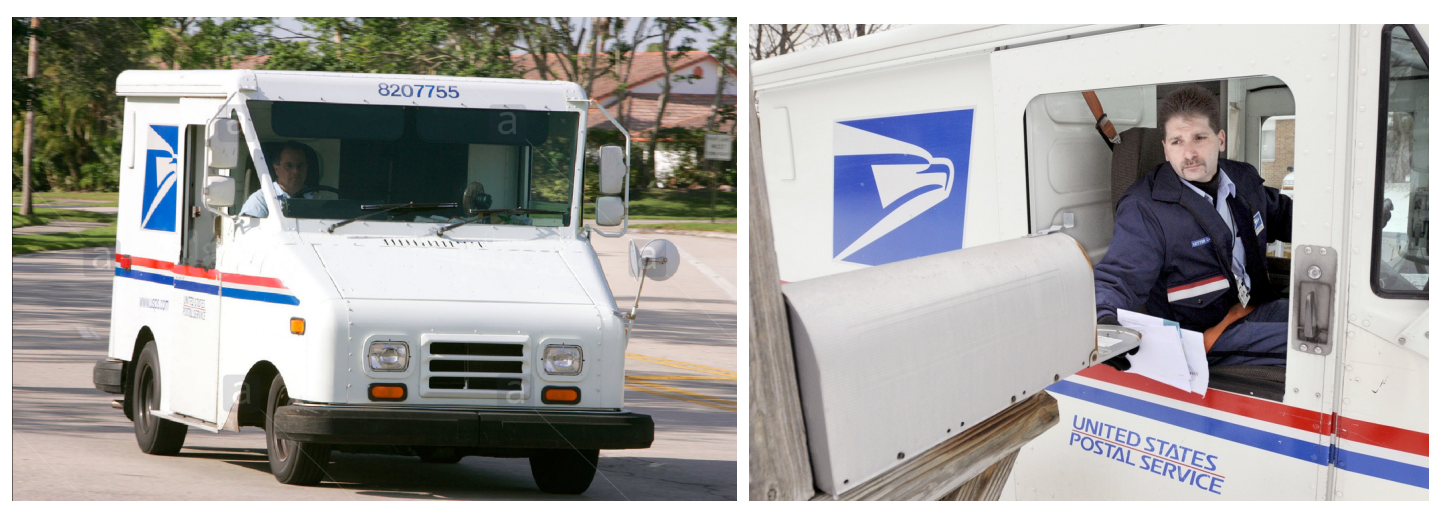

Figure 3: US Postal Servic Truck 
one side and holes on the other side, some says men's and women's are opposite. However, thousands year ago, in ancient China, the design was not as unified. The way to close a coat, whether left side on top of right, or right side on top of left, is one of the major distinctions of the Han dynasty. The majority of people lived in central china and minority in the frontier, the $H u$, . In 307 AD, King Wuling of Zhao commanded Han, his people, to wear $H u$ styled cloths for better shooting and horse riding in battle. This event is called $\mathrm{Hu} \mathrm{Fu} \mathrm{Qi} \mathrm{Se.} \mathrm{[7]}$

\subsubsection{Chirality in chemistry and materials science}

Chirality in chemistry was experimentally proved by Louis Pasteur in 1848, who found tartaric acid forms different shaped of crystals [8] [9] [10], (see figure 4). By hand he separated the two different shape of the crystals. He found that both of the two kinds of crystals are optically active, and that they can rotate polarized light by the same amount, but in opposite orientation. He was able to show that the optical activity is related to the shape of the crystals. He predicted that the shape of the crystals was related to the internal arrangement of the atoms making up the molecules of the compound and that this was responsible for twisting the light. He called this phenomenon molecular chirality, which indicates the relation between optical rotation 
and chemical structures.

Because of his significant contribution, people nominate him as father of sterochemistry in Quora. However, it is a pity that Pasteur did not really understand sterochemisty at that time, so he could not explain the origin of the two handness in the way of symmetric theory. The method he used to separate the two handness Tartaric acid was by hand selecting the crystals based on their different ( and mirror image) shapes. Since he could only separate the crystal by crystals shape appearance, he failed to recognize that mallic acide is also chiral. Later in 1874, Joseph Achille Le Bel and Kacobus Henricus van't Hoff announced their hypothesis about the bounding tetrahedral structures. [11] Their theory explained why only two isomers are exist, and why they are exactly the mirror images of each other, and why it is hard to convert from one to another. Thus, chemists were able to connect optical activity with the three dimensional molecular structure.

Another interesting example is people can tell the different smell of caraway seeds and spearmint leaves. Actually, the caraway-like smell is caused by R-carvone, and S-carvone causes the spearmint-like smell. The R-carvone and S-carvone have very similar chemical structure, the only difference are their 3D chemical structures are mirror images of each other. See Figure 5. T. Leitereg published this finding [12] in 

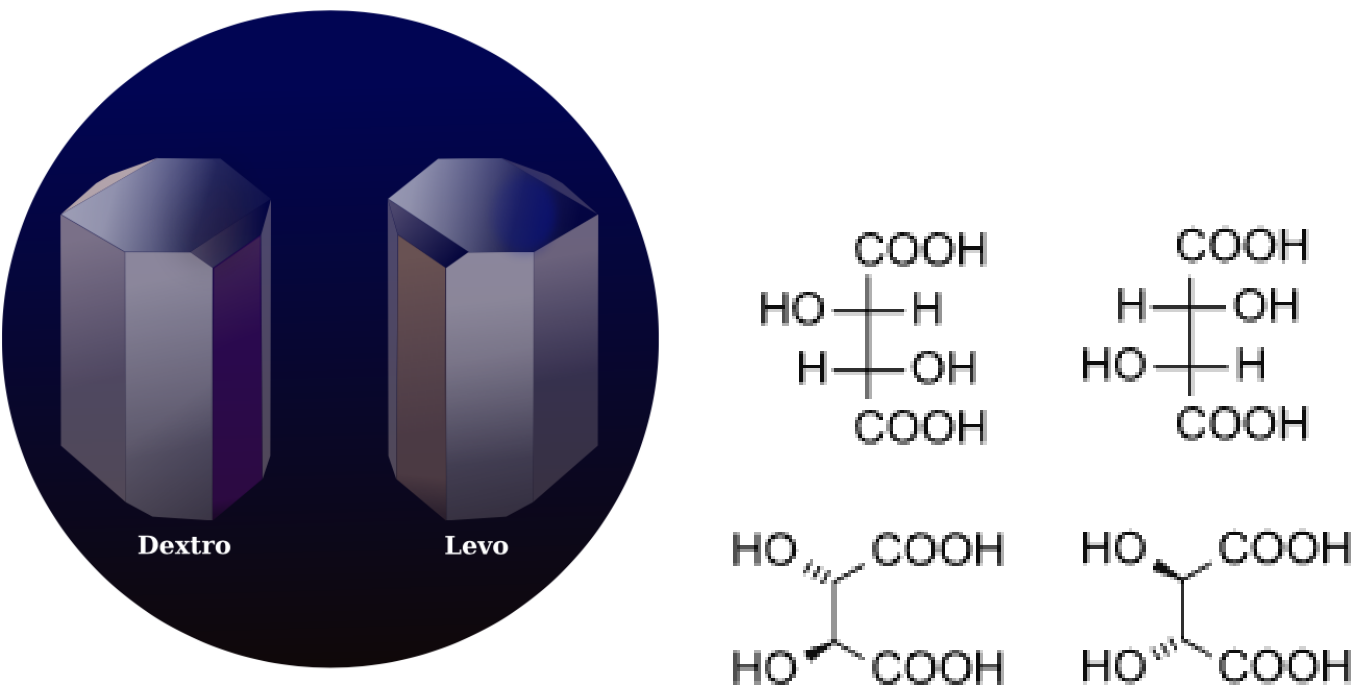

Figure 4: The (2R,3R)- and (2S,3S)- tartrates were isometric, non-superposable mirror images of each other. 


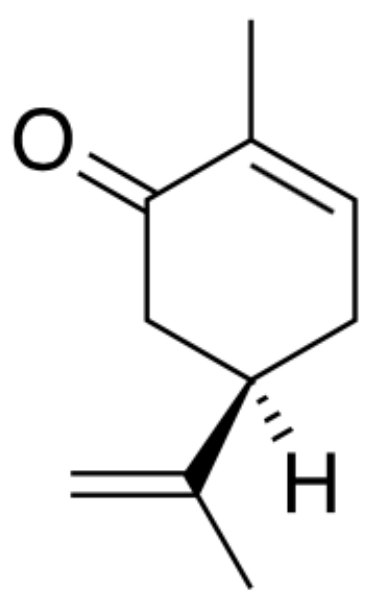

(R)

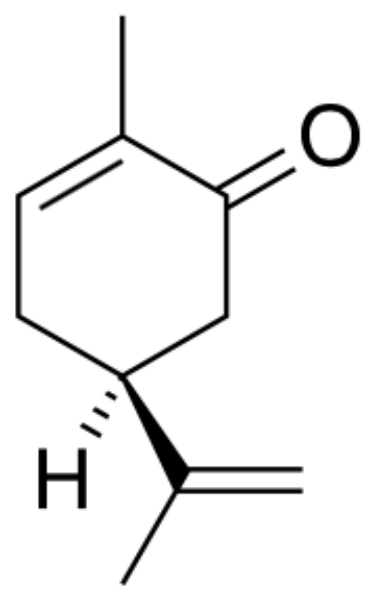

(S)

Figure 5: R-carvone smells like caraway, and S-carvone smells like spearmint 
1971. 28 years later, M. Laska and coworkers figured not only human but also other animals, like squirrel monkeys, can distinquish the smell of the two carvones [13]. In their experiment, they also found squirrel monkeys could also tell odors from Rlimonene (orange-like smell)and S-limonene (turpentine-like smell), ), just as humans can. [14] [15].

Relevant to this thesis, the first liquid crystal found was chiral. In 1888, Austrian botanical physiologist Friedrich Reinitzer was working on various derivatives of cholesterol, see Figure 6 [16] . He observed the two melting points in cholesteryl benzoate. At the lower melting point, the crystal melts into a cloudy fluid, later found to be optically active. Then, at the second melting point, this cloudy fluid becomes clear. Later this cloudy fluid, existing between the two melting point was identified as a cholesteric liquid crystal.

Chiral liquid crystasl have the subject of research since the day they were discovered citeFriedel1922 [17]. Some liquid crystal materials are themselves chiral, so single substances can form the cholesteric liquid crystal phase, such as hydroxypropyl cellulose and cholesteryl benzoate. However, chirality of the liquid crystal material is not required. The appearance of a chiral liquid crystal phase can be achieved by dissolving chiral additives into a non- chiral liquid crystal host. One typical chiral 


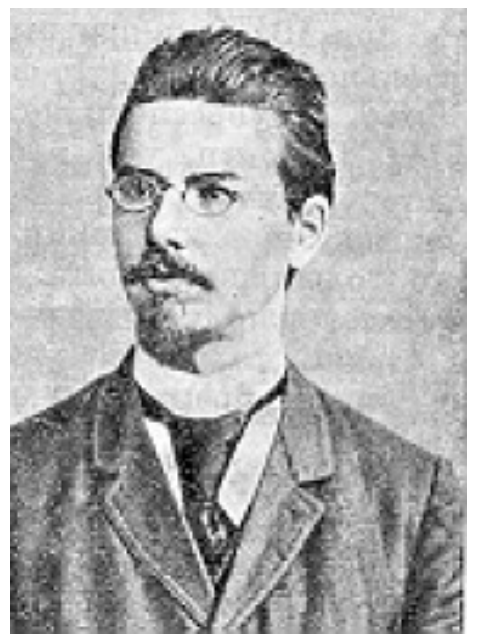

Figure 6: Friedrich Reinitzer (1857-1927) 


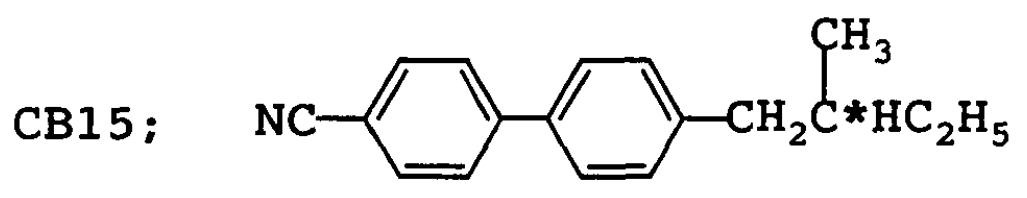

Figure 7: Molecular structure of (S)-4'-(2-methylbutyl)[1,1'-biphenyl]-4-carbonitrile (CB15) 


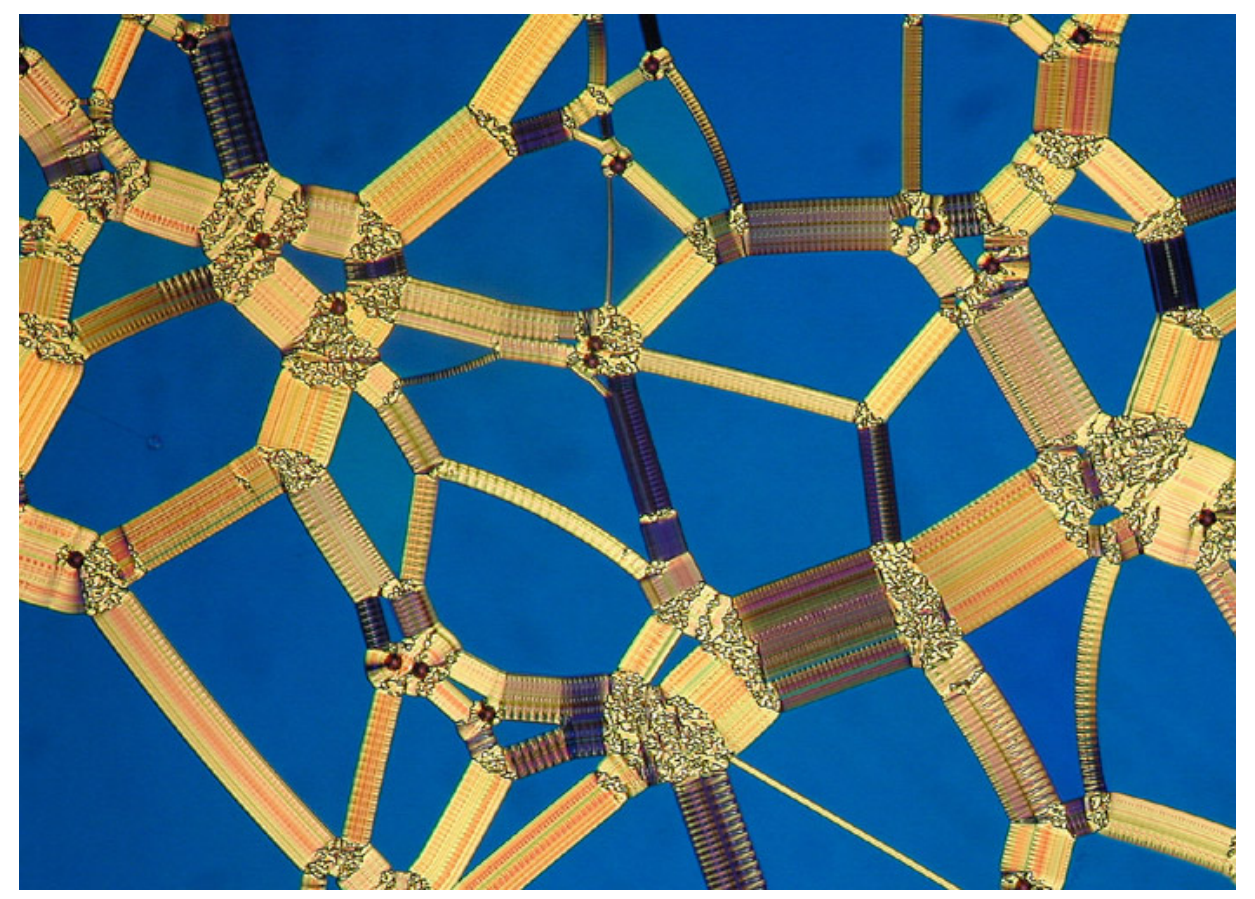

Figure 8: CB15, a cholesteric liquid crystal used in the manufacturing of liquid crystal displays, at 100-times magnification. Image by Dr. Christian Bohley, Otto-vonGuericke-University of Magdeburg. 
compound used in liquid crystal research and the display industry is CB15, (S)-4'-(2methylbutyl)[1,1'-biphenyl]-4-carbonitrile, see figure 8. The chiral carbon, also called chiral center, inside the molecule is connected to four different functional groups. The chiral carbon's handness determines the liquid crystal's handness. In the case of CB15, the chiral carbon is (S)-oriented, and CB15 is left-handed. [18]

Some chiral liquid crystals doesn't have either a chiral carbon, or a chiral center. Rather the geometry of the molecular structure produces a chiral conformation, and restricts switching to the other confirmations. Most of theses types of liquid crystal molecules have multi-aromatic rings in their core.

Cholesteric liquid crystal materials are used in displays. They help determine the sense of twist in the traditional twist nematic or supertwist nematic (TN/STN) liquid crystal displays, blue phase liquid crystal display and advance super view display techniques (chiral vertical alignment). Cholesteric liquid crystals are used in the popular writing tablet, the Boogie Board. By dispersing a cholesteric liquid into polymer network, Kent Display Inc. was able to make a pressure sensitive, bistable, flexible and wide- viewing-angle reflective liquid crystal display by sandwiching the material between two plastic substrates. 


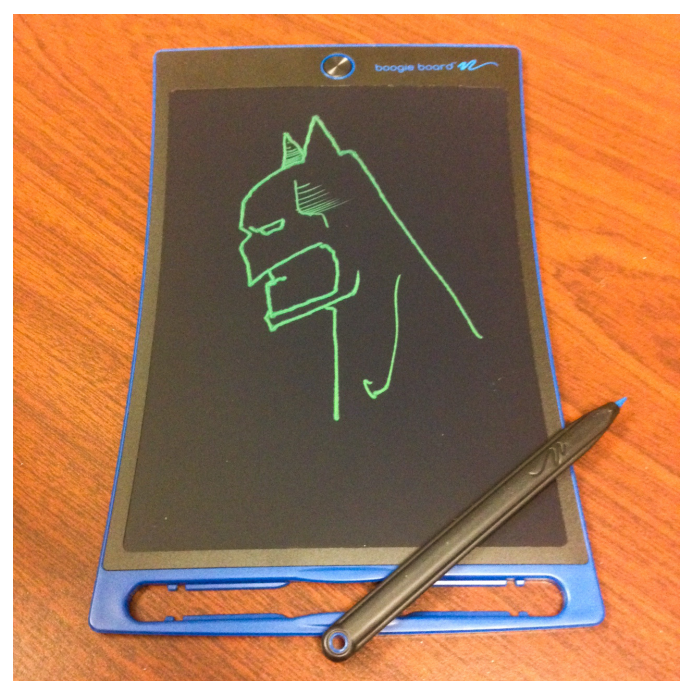

Figure 9: Boogie Board Jot 8.5 eWriter. 


\subsection{Thesis Overview and Organization}

In this thesis, I present my work to achieve the goal of understanding the relation between molecular level conformation and liquid crystal chirality.

In Chapter 1, we introduced the phenomenon of chirality and reviewed the history of chirality in early time, among artists, philosophers; and human understanding. We tried to give the reader an intuitive concept and show that Chirality is a common entity, which we can find everywhere in our daily life, while it remains an exciting topic for scientists.

In Chapter 2, we review chirality in liquid crystals. We cover the basic concepts of liquid crystal structure and focus on cholesteric liquid crystals and chiral dopants. We also introduce the concept of helical twisting power, which is a measure of ability of a chiral additive to twist liquid crystal into a helical structure. A list of common helical twisting power measurement methods is also studied.

In Chapter 3, we mainly focus on the molecular conformation induced chirality. Using molecular dynamic modeling, we model helicity variation based on molecular 
structure. This method is used in the following chapters to predict the dependence of the helical twisting power of a chiral dopant on temperature.

In Chapter 4, we present experimental results that evaluate the helical twisting power temperature dependence of a series of isosorbide chiral dopants. Although these dopants have a similar core structure, we found very different helical twisting power temperature dependences. Through UV-Vis spectroscopy, NMR spectroscopy and molecular simulation, we demonstrated that conformational changes can explain many of the observed responses of helical twisting power to temperature.

In Chapter 5, we turn our focus to the interaction between chiral dopants and liquid crystals. Not only the conformational change in the chiral dopant itself, but also its impact on liquid crystals. We postulate that the way the chirality of a chiral dopant propagates through the liquid crystals is based on liquid crystal chirality change.

Finally, Chapter 6 concludes the thesis with a summary of contributions and discussions for future research. 


\section{CHAPTER 2}

\section{Cholesteric Liquid Crystals and Chiral Dopants}

\subsection{Liquid Crystals and Liquid Crystalline Phases}

Liquid crystal is a state of matter exists between the solid crystal and isotropic liquid phases. It has properties of both liquids and crystals. For example, it can flow, and each molecule might position randomly inside the bulk like a liquid but the molecules retain some of the orientation characteristic of a crystal [19] [20] [21] [22]. Its molecular order makes many physical properties of this material orientationally dependent. These physical properties include dielectric permittivity, magnetic susceptibility, thermal and electrical conductivity and light transmittance, etc. These special properties have drawn the interests from the greatest scientists in the world. In 1991, Pierre-Gilles de Gennes (See Fig 10) was awarded the Nobel Prized in Physics,

for his contribution to the theoretical understanding of liquid crystals as well as his contribution in polymers, superconductors and magnetic materials. 


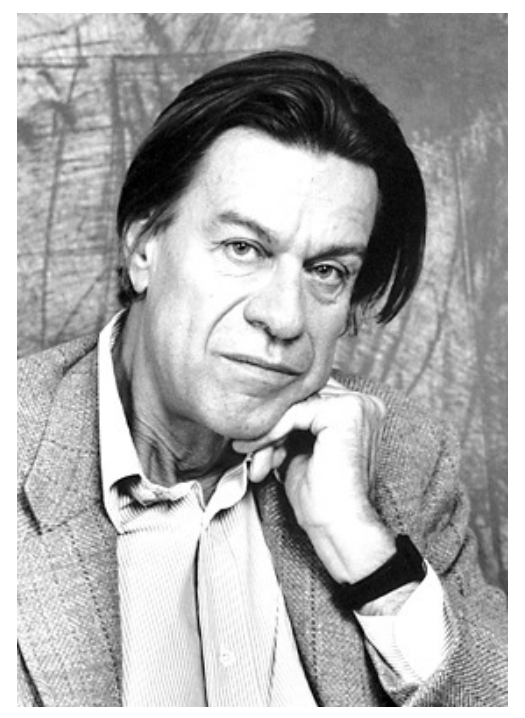

Figure 10: Pierre-Gilles de Gennes (1932-2007) 
There are many liquid crystal phases, which differ in symmetry and degree of order. Different liquid crystal phases appear different when viewed using a polarizing microscope and a polarized light source. [23] Another distinction between liquid crystal types is made, between thermotropic liquid crystals and lyotropic liquid crystals. Lyotropic liquid crystals usually consist of two or more materials, a solute and a solvent. The solutes are typically amphiphilic compounds, or semi-flexible polymers. When changing the concentration or temperature of the solution, lyotropic liquid crystal phases appear. The most common lyotropic liquid crystalline system is soap. Other lyotropic liquid crystals systems are found in living systems, for example cell membranes (see Figure 11) [24] [25] . The study of lyotropic liquid crystals are extremely important for understanding biological membranes and drug delivery.

Thermotropic liquid crystals are usually low molecular weight organic molecules. Over a certain temperature change, these molecules exhibits one or more liquid crystalline phases. At higher temperatures the thermal energy destroys the orientational order and the materials becomes an isotropic liquid.This transition temperature is called the clearing temperature, and is called $T_{N I}$. when the transition is from the nematic $N$ phase, to the isotropic phase $I$. This phase transition is a first order phase transition. 


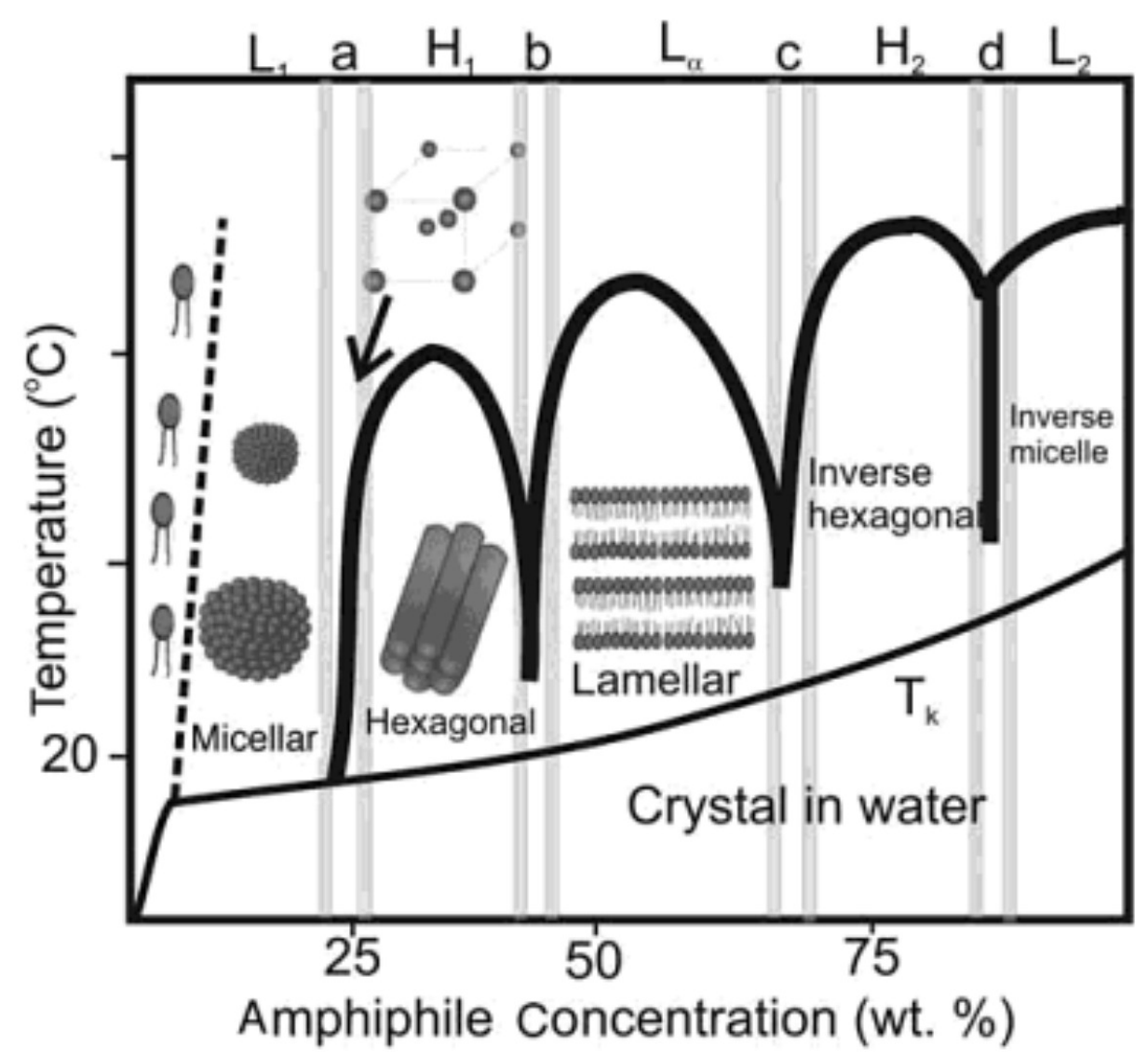

Figure 11: Phase Diagram for a typical soap-water system 


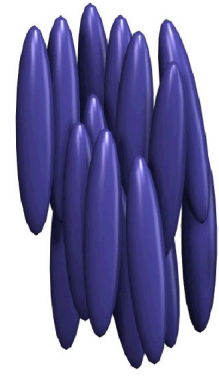

(a)

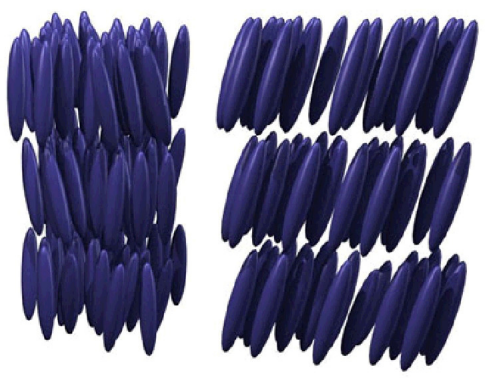

(b)

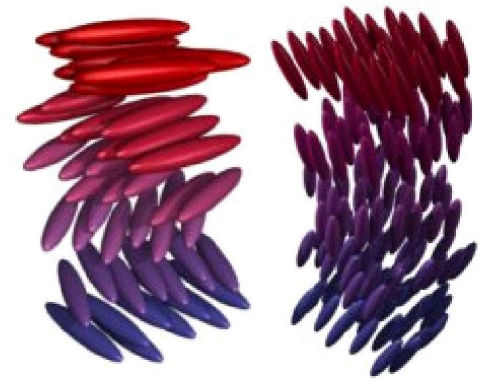

(c)

Figure 12: Schematic representations of (a) a nematic LC phase, (b) smectic LC phases oriented along (left) and away (right) from the normal of the layer, and (c) a chiral LC phase. 
The common liquid crystal phases are Nematic, Smectic and blue phases. The smectic $\mathrm{C}^{*}$ where the molecules are oriented away from the normal to the layers and blue phases are chiral, see Figure 12. In the nematic phase, the liquid crystal molecules' centers of mass are randomly located: their is no positional order. In the nematic phase, the molecules self-align maintaining a long-range directional order. In a nematic aligned in a single direction, the molecues are all roughly parallel and pointing at the same direction and its inverse. The average orientation of their long axis is defined as the liquid crystal director. Nematics in general can flow like liquids. Aligned nematic liquid crystals are widely used in liquid crystal displays.

Unlike the randomly positioned nematic phase, smectic liquid crystal phases have a layered structure. Molecules in this liquid crystal phase are arranged into layers. In this phase, molecules can move freely inside the layer, but it is hard to move between layers. So the liquidity is confined in a 2D surface. The cholesteric phase and blue phase are both chiral liquid crystal phases. These phases will be covered in the next section. 


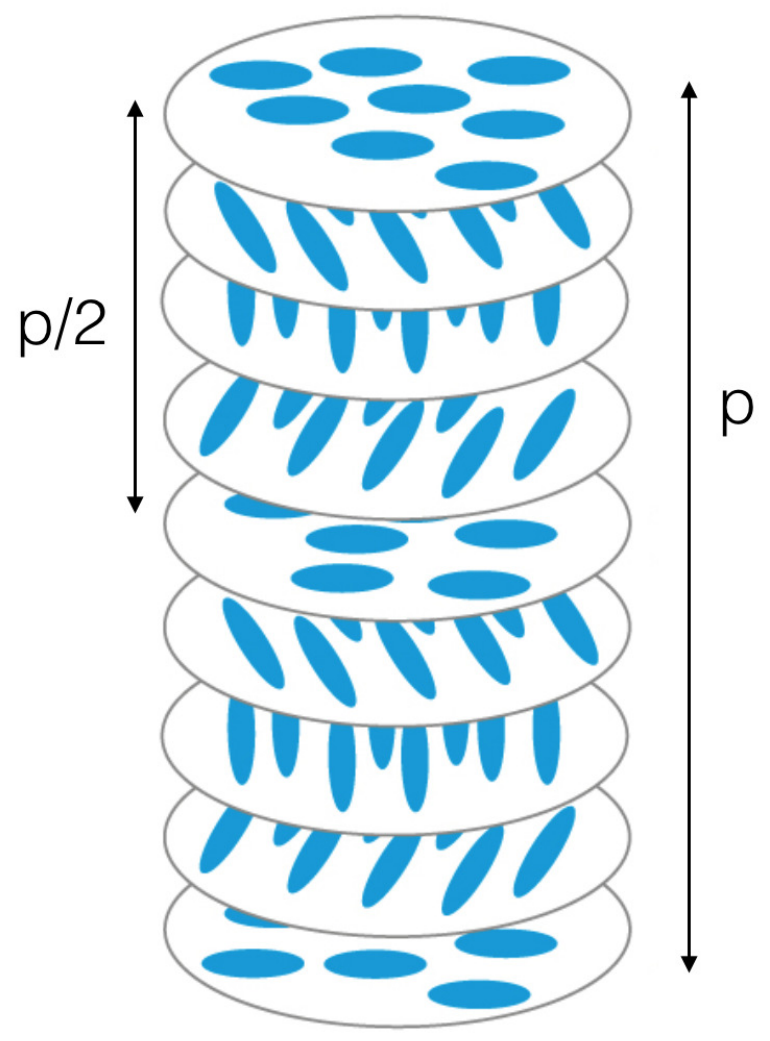

Figure 13: Cholesteric liquid crystal phase. 


\subsection{Chiral Liquid Crystals and Helical Twisting Power (HTP)}

The cholesteric phase was first observed in cholesterol derivatives. It is also called the chiral nematic phase. In the cholesteric phase, the liquid crystal director rotates in the plane that is vertical to helical direction, see Figure 13. If observing from top to bottom, the molecules undergo a full circle and go back to original direction. The distance between the original molecular direction and after $360^{\circ}$ rotation is called chiral pitch, noted as $p$. And $p / 2$ is also commonly used to refer to the distance corresponding to a $180^{\circ}$ rotation.

The nematics phase is locally uniaxial with $C_{\infty}$ axis coincident with the director. Since the director $n$ and $-n$ are equivalent in nematic phase, it has $D_{\infty h}$ symmetry. While in the cholesteric phase, the helical structure breaks mirror symmetry, but leaves the rotational symmetry.

The blue phase has a regular three-dimensional cubic structure of defects with lattice periods of a few hundred nanometers. This structure is an optically isotropic liquid crystal phase [26]. The blue phase has double twist cylinders, which are ordered in cubic arrangements, the blue phase can be seen as a fluid lattices of defects. 


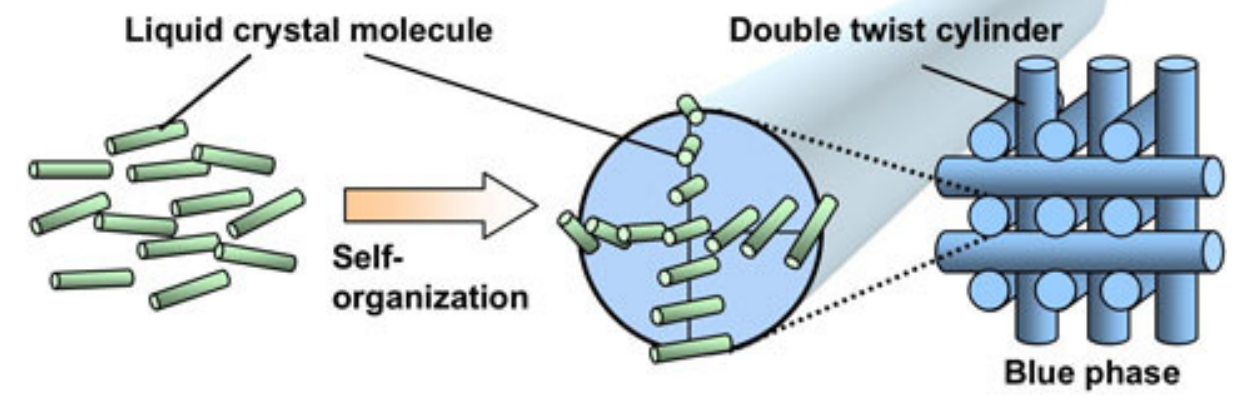

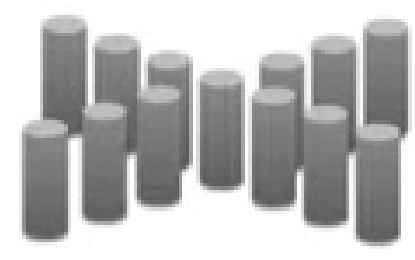

$\mathrm{N}$

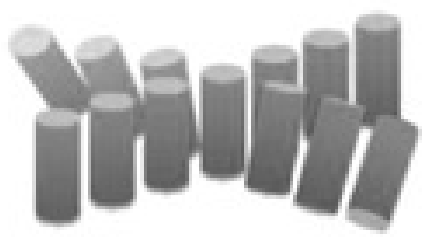

$N^{*}$

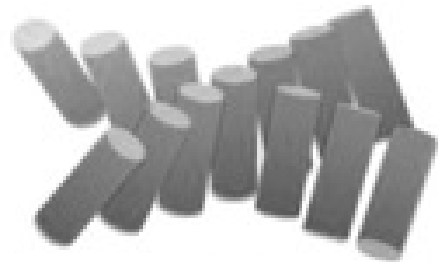

BP

Figure 14: Top: Blue Phase double twist structure; Bottom: Nematic Phase (N), Chiral Nematic Phase $\left(\mathrm{N}^{*}\right)$, Blue Phase (BP) 
It was theoretically predicted in 1981 that these phases possess icosahedral symmetry similar to quasicrystals [27]. For a long time, blue phases were considered as stable only under very special conditions. Since 2005, people have reported possible blue phase mixtures with a wider temperature range [28] [29]. Later, Kikuchi and coworkers reported that inducing polymer into blue phase system could efficiently stabilize the blue phase at room temperature, ranging from $260 \mathrm{~K}$ to $326 \mathrm{~K}$. [30]

Another chiral liquid crystal phase is the Smectic $\mathrm{C}^{*}$ phase. The smectic $\mathrm{C}^{*}$ phase is very similar to the cholesteric phase, it also has a helical structure. Meanwhile, like other smectic phases, it has a clear layered structure. The molecules are tilted at an angle to the layer normal. In each layer the tilt angle is roughly the same, but the pointing in a direction that gradually rotates around the layer normal. Most smectic $\mathrm{C}^{*}$ phases exhibit ferroelectric properties [31]. As long as the directer rotate along the cone structure (see in Figure 15), the material is in a stable state. This enables the bistability and fast switching speed of the smectic $\mathrm{C}^{*}$. [32]

To better understand chiral nematic systems and even predict their behavior, it is important to have a theoretical description, which can be complemented by modeling and simulations. Such system could be described and modeled at very different scales: 


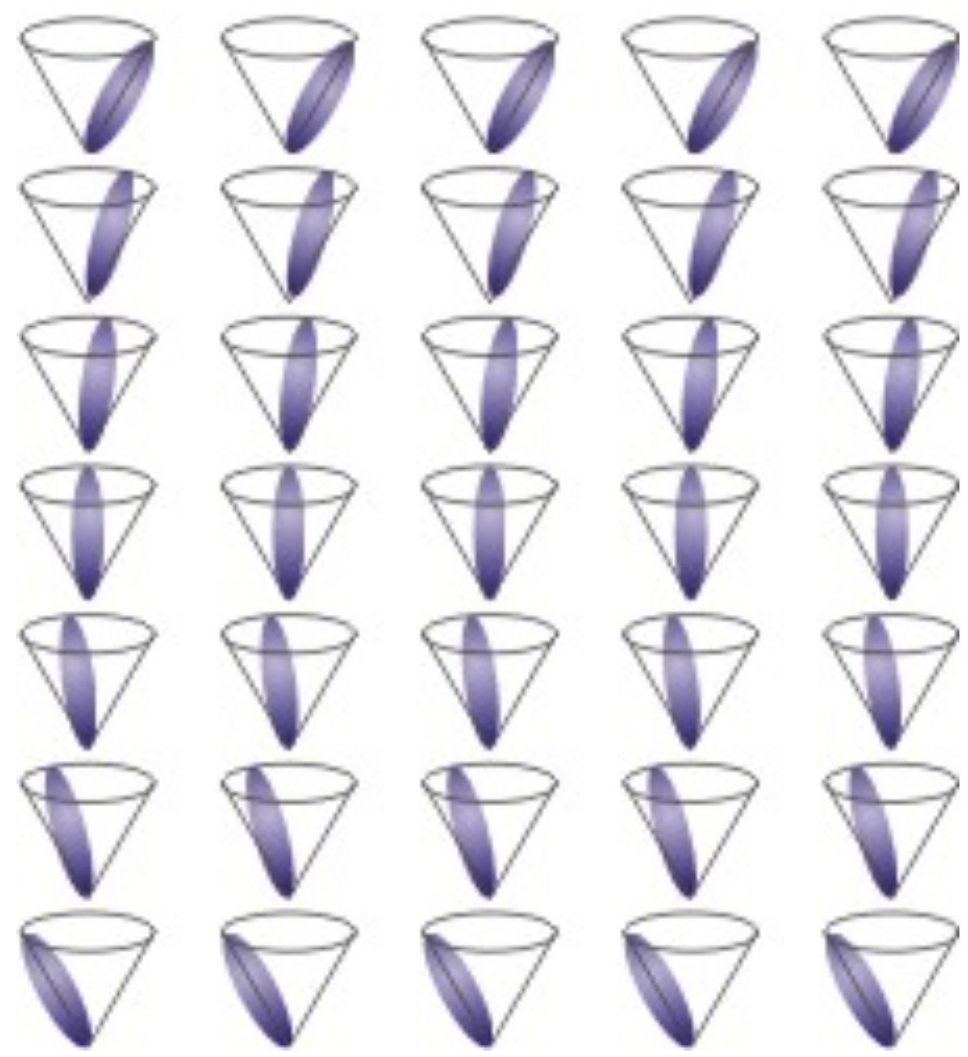

Figure 15: Sketch of layered organization of smectic $\mathrm{C}^{*}$ phase 
from molecular to macroscopic scales. Microscopic scale simulation could provide detailed information [33], especially, in confined systems [34] [35], but are normally performed for small scales and take a lot of computer time. On the other hand, thermodynamic calculation on macroscopic scales could provide a general understanding and a physical picture [36]. When considering all three basic deformation modes in bulk nematic phases, the liquid crystal free energy density could be written as

$$
F_{d}=\frac{1}{2} K_{11}(\nabla \cdot \vec{n})^{2}+\frac{1}{2} K_{22}(\vec{n} \cdot \nabla \times \vec{n})^{2}+\frac{1}{2} K_{33}(\vec{n} \times \nabla \times \vec{n})^{2}
$$

Here $K_{11}, K_{22}, K_{33}$ are the diagonal elements of the elastic tensor. They are deformation elastic constants, which are correlated to splay $\left(K_{11}\right)$, twist $\left(K_{22}\right)$ and bend $\left(K_{33}\right)$, respectively. In 1958 F.C. Frank extended the nematic free energy expression to the chiral nematic case. [37]

$$
F_{d}=\frac{1}{2} K_{11}(\nabla \cdot \vec{n})^{2}+\frac{1}{2} K_{22}(\vec{n} \cdot \nabla \times n \geq q)^{2}+\frac{1}{2} K_{33}(\vec{n} \times \nabla \times \vec{n})^{2}
$$

Here $q$ is the helix wavenumber, $q=2 \pi / p$. Since in the chiral nematic phase, only the twist deformation contributes into the total free energy.

Another way of the free energy expression is

$$
F_{d}=k_{2} q+\frac{1}{2} K_{22} q^{2}
$$


Where $k_{2}$ represents chiral strength, which is determined by the chirality of intermolecular interactions. When free energy reaches the equilibrium state, $q$ can be calculated

$$
q=-\frac{k_{2}}{K_{22}}
$$

Here $K_{22}$ is the elastic torque opposing twist deformation. The larger the $K_{22}$, the smaller the $q$. And the larger the chiral strength is, the bigger the $q$, and the tighter the pitch. When $K_{22}$ is much bigger than $k_{2}, q \rightarrow 0, p \rightarrow \infty$, it becomes the nematic phase. So the nematic phase can be seen as a special case of the cholesteric phase, when the pitch is $\infty$.

\subsection{Helical Twisting Power}

In general, there are two kinds of cholesteric liquid crystalline phase systems: one is formed by chiral liquid crystals. In this case, the liquid crystal is chiral itself. A typical chiral liquid crystal molecule is (S)-4'-(2-methylbutyl) [1,1'-biphenyl]-4-carbonitrile (CB15, see figure 7). As mentioned earlier in Chapter 1, in this molecule, there is a 
chiral carbon connected to four different functional groups. The chiral carbon's handness determines the liquid crystal's handness. In the CB15 case, the chiral carbon's chirality is $(S)$, and CB15 is left-handed. [18]

However, chirality from the liquid crystal itself is not always required. Chirality can be induced by adding chiral additives into a non-chiral liquid crystal material and form a mixture system. This is actually more common way, as a much wider range of liquid crystal materials can be applied in this approach, as long as a chiral additive can be dissolved in the non-chiral liquid crystal host.

Sometimes, even a small amount of chiral additives can induce a large chirality change into the liquid crystal host; while other times a larger amount of chiral additives are required to reach the same pitch and chirality. So scientists need a way to describe the ability of chiral additive to make liquid crystal forming helix structure. Experimentally, one finds at low concentration, pitch is inversely proportional to concentration of the chiral additive.

$$
p \cdot c=\text { constant }
$$

Here $\mathrm{p}$ is the pitch of liquid crystal helical structure, and $c$ is the mole fraction of the chiral additive. We can define the ability of a chiral additive ton induce the liquid 
crystal into a helical structure as the helical twisting power. The stronger the helical twisting power is, at the same concentration, the tighter pitch. Experimental work points to the fact that different dopant molecules have widely different abilities to twist a nematic phase, and this is normally characterized by defining a macroscopic helical twisting power.

$$
H T P=\frac{1}{p \cdot c}
$$

HTP varies for different chiral additive materials. And for even the same chiral additive, when adding into different liquid crystal, HTP can vary. [38]

Normally a small amount of chiral additive can induce a helical pitch on the order of microns. Certain display applications requires a shorter pitch length, on the order of wavelength of visible light. Since using chiral additives in large concentration may lead to high cost and degrade the liquid crystal electro-optical performance, high helical twisting power (HTP) materials need to be introduced. Typically, high HTP compounds show a larger temperature dependence than samll HTP compounds. Although ideally, a small temperature dependence is preferable. Because of the high cost and high labor required to synthesize and characterize chiral dopants, understanding the mechanism of chiral induction is important to provide better guidance in selecting the materials to be synthesized. 


\section{$2.4 \quad \theta$-cell Measuring Helical Twisting Power}

Once understanding the concept of helical twisting power of cholesteric liquid crystal system, we would like to quantitatively determine the helical twisting power by performing measurements. The common method of determining the HTP is by measuring the pitch, then HTP can be calculated using Equation 6. The value of p can be determined in several ways. Some common methods are discussed below: reflectivity method [39] [40] [41], wedge cell method [42] [43], finger print, method, Bragg scattering method [44] [45], and some recent discovered methods, like using a TN cell [46] [47] [48] and the $\theta$-cell method [49] [50] [51] [52]. The latter one is particularly good for measuring liquid crystal with centimeter range pitch.

The $\theta$-cell method was originally proposed by Seong-Woo Suh and coworkers in 1990s [49], and recently reinvented by Vasnetso and coworkers [50] [51]. The LC cell is made with one homogeneously rubbed surface and one circularly rubbed surface, as seen in Fig 16 [52]This special geometry produces a disinclination line passing though the cell, with two domains corresponding to the two different twist orientations. 

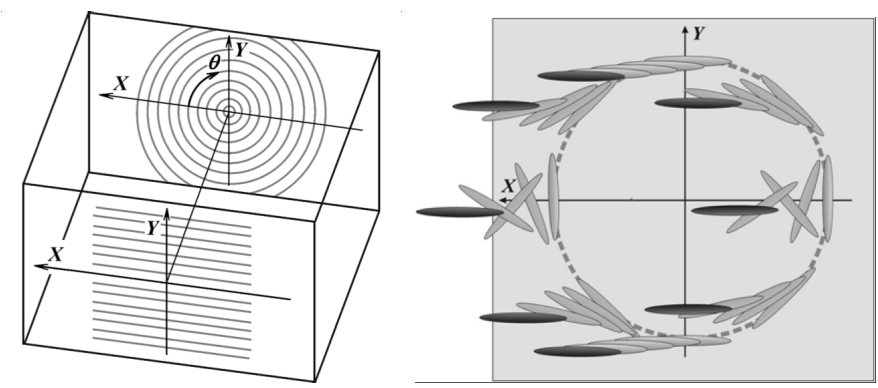

Figure 16: Left:The rubbing of substrates:front substrate with linear alignment, rear substrate with circular alignment. Right: The orientation of LC molecules, a view from the linear to the circular rubbing 
Assuming an achiral liquid crystal case, the cell is divided into four domains, in the first and the third quadrants the liquid crystal director twists clockwise; and in the second and forth quadrants, the liquid crystal director twists counter-clockwise. Disclination line form along the alignment direction of the planer aligned front substrate. Now consider what happens when the liquid crystal is doped with a chiral dopant, assuming the doping amount is small, so that the pitch is still larger than cell thickness, $l$, the disclination line rotate will by angle $\delta$,

$$
\delta_{+,-}=\frac{2 \pi l}{p_{+,-}}
$$

where + , - indicate the sign of the chirality. Clockwise rotation is related to the right-handed twist; and the counterclockwise rotation is related to the left-handed twist.

When the doping amount increases, the pitch gets smaller. Once the pitch is smaller than cell thickness, liquid crystal director can rotate multiple pitch lengths before reaching the other side of the substrate. In this case, pitch could be written as

$$
p_{+,-}=\frac{2 \pi}{2 \pi n+\delta_{+,-}}
$$


Where $\mathrm{n}$ is an integer, that indicates the number of disclination line rotations. Since the disclination line is a stationary topological defect, which is visible, and can be easily and precisely evaluated under optical microscope, the $\theta$-cell method allows precisely measurements of the pitch over a very wide range $p=1-10^{4} \mu m$ when $p>l$. However, when $p<l$, the measurement accuracy is very sensitive to the cell thickness.

One interesting thing is, when measuring pitch in a $\theta$-cell, the disclination line sometime exhibits an $S$ - shape rather than a straight line. This is caused by cell thickness non-uniformity when using capillary filled cells. The adhesive used to hold the cell together usually changes in volume during curing. This process generates stress forces that are trapped inside the liquid crystal cells, and form non-uniform cell thickness. Usually there is a smaller cell thickness at the center. In this case, the rotation angle of the disclination increase when moving away from the center of the cell, as explained through in Eq 7, and shown in Fig 17 .

Luckily, there is an easy fix for this kind of problems. After filling in the cells, just simply heat the liquid crystal cells into the isotropic phase, then slowly cool down to room temperature, the abnormal S-shaped disclination line could be "fixed" into straight lines, as seen in figure 18, the photos show $\theta$-cells filled with $5 \mathrm{CB}$ doped 


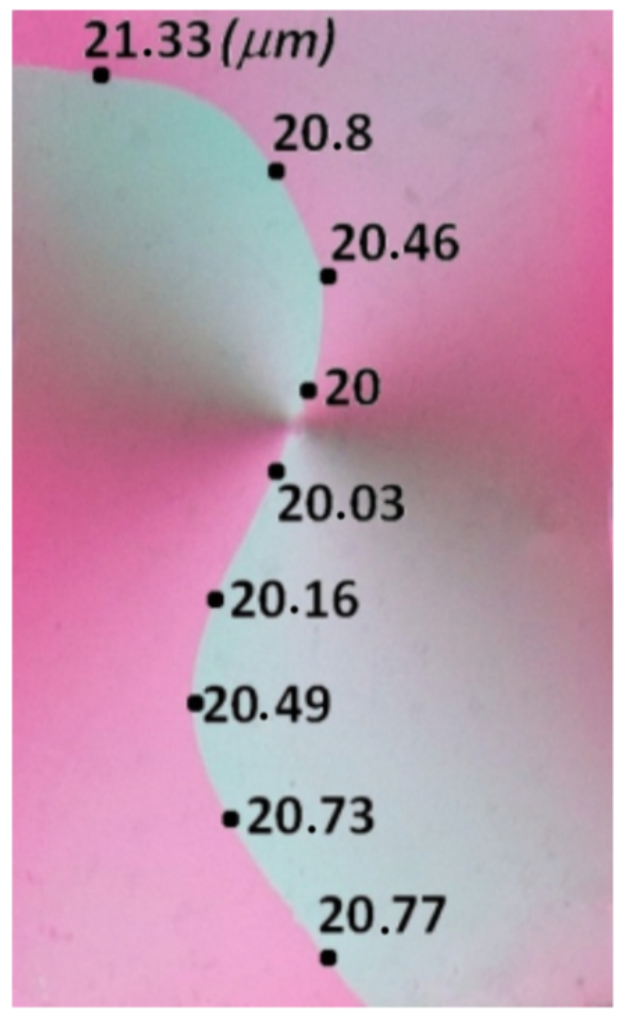

Figure 17: Photograph of the cell with inhomoge- neous thickness in crossed polarizers. The cells filled with composi- tion 5CB and S-811: $c=3.7 \times 10^{-3}$. The numbers indicate the local thickness of the cell. 


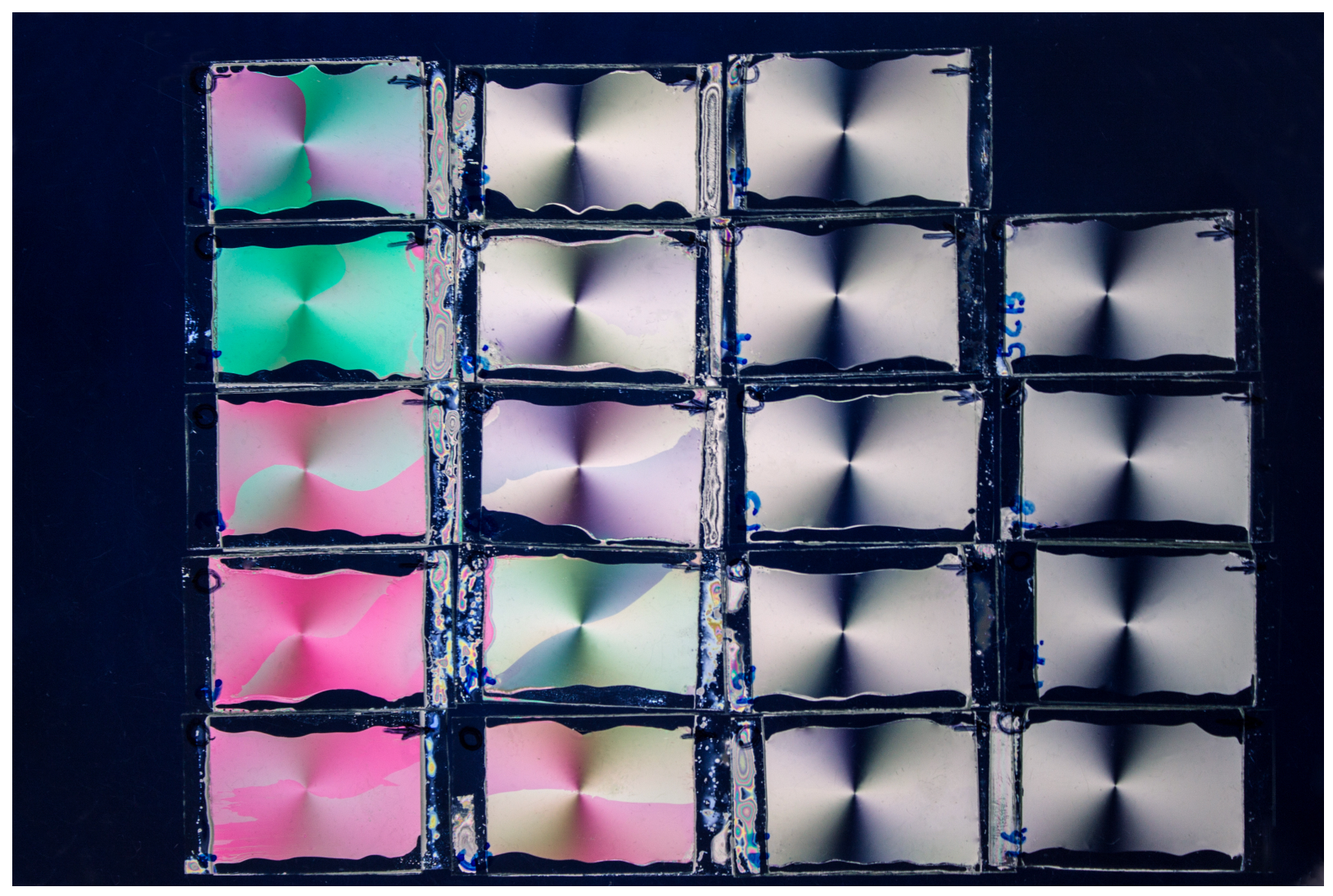

Figure 18: Photograph of 5CB doped with CD6 at different concentration in $\theta$-cells. 

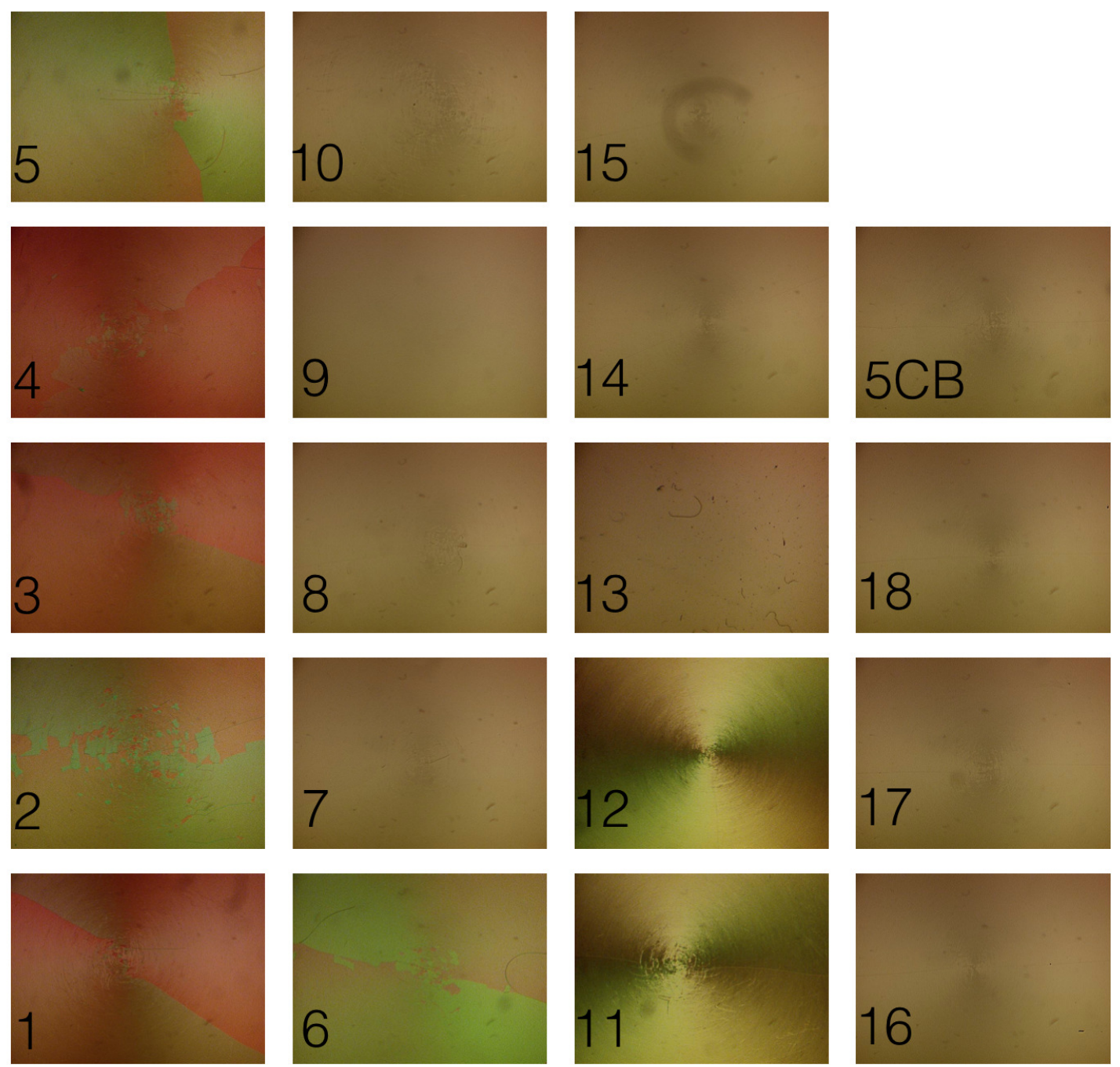

Figure 19: The same cells heated up to isotropic phase, then slowly cool down to room temperature 
different concentration of chiral dopant right after filling. The $S$ - shaped disclination lines can be seen clearly. And in figure19 shows the same samples heat up to isotropic state, then slowly cool down to room temperature. The heating and cooling process relax the stress inside the cells. In the the stress released cells, the $S$ - shaped disclination lines relaxed to straight lines. The sample number and the concentration of chiral dopant is listed in table 2

Combining Equation 6 and 7, one can easily get,

$$
\begin{gathered}
\delta_{+,-} / c=\text { constant } * H P T \\
\text { constant }=2 \pi l
\end{gathered}
$$

Eq 9 means that, at low concentration, once the cell thickness is fixed, the ratio of twisted angle over chiral dopant concentration depends on helical twisting power. So once we plot the twist angle of the disclination lines in test $\theta$-cells over the chiral additive concentration, the slope $=71.095$ could be calculated by linearly fitting. Since cell gap, $l$, is known as $20.1 \mu m$, HTP for R811 in 5CB could be calculated as $56.32 \mu \mathrm{m}^{-1}$. Even when concentration goes as low as $1.25 \times 10^{-6}$, this value stays the same. 
Table 2: Sample number and the concentration of R811(chiral dopant)

\begin{tabular}{c|c}
\hline Sample No. & wt\% of R-811 \\
\hline Sample-1 & $5.25 \mathrm{E}-01$ \\
\hline Sample-2 & $4.50 \mathrm{E}-01$ \\
\hline Sample-3 & $4.30 \mathrm{E}-01$ \\
\hline Sample-4 & $3.71 \mathrm{E}-01$ \\
\hline Sample-5 & $3.29 \mathrm{E}-01$ \\
\hline Sample-6 & $2.61 \mathrm{E}-01$ \\
\hline Sample-7 & $2.25 \mathrm{E}-01$ \\
\hline Sample-8 & $1.74 \mathrm{E}-01$ \\
\hline Sample-9 & $1.25 \mathrm{E}-01$ \\
\hline Sample-10 & $6.63 \mathrm{E}-02$ \\
\hline Sample-11 & $4.20 \mathrm{E}-02$ \\
\hline Sample-12 & $2.51 \mathrm{E}-02$ \\
\hline Sample-13 & $1.50 \mathrm{E}-02$ \\
\hline Sample-14 & $5.00 \mathrm{E}-03$ \\
\hline Sample-15 & $2.50 \mathrm{E}-03$ \\
\hline Sample-16 & $5.00 \mathrm{E}-04$ \\
\hline Sample-17 & $2.51 \mathrm{E}-04$ \\
\hline Sample-18 & $1.25 \mathrm{E}-04$ \\
\hline
\end{tabular}



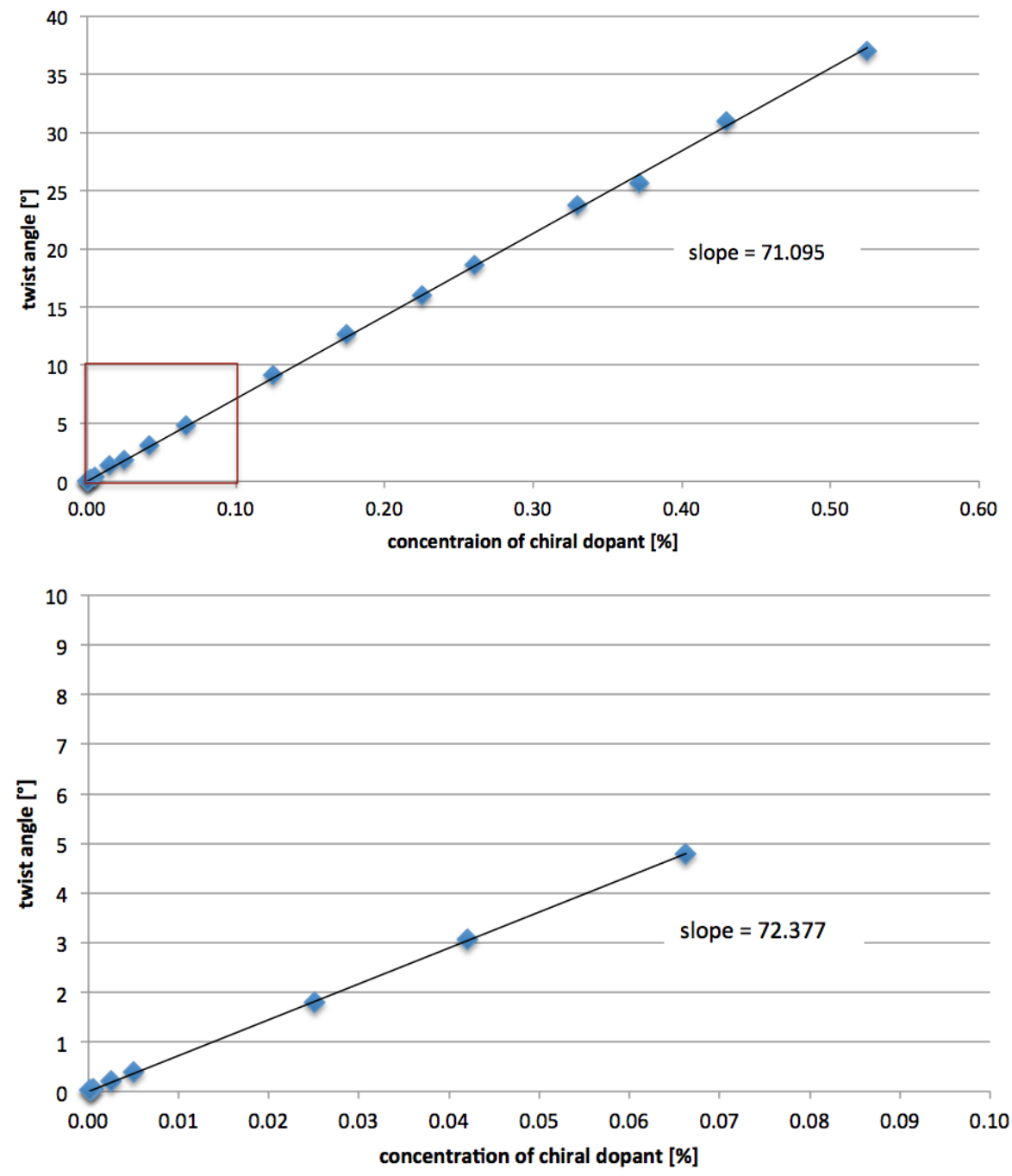

Figure 20: Twist angle of disclination line in $\theta$-cells vs concentration of the chiral dopant in liquid crystal host. 
Assuming the chiral dopant is uniformly distributed inside the liquid crystal host, and they are positioned on a three-dimensional lattice structure, although this is not the case in liquid crystals. From the concentration, we can calculate that the chiral dopant molecules are around 100nm apart from each other in the liquid crystal host. This means the chiral dopant has an impact on the liquid crystal host in $0.1 \mu m$ range, and makes them form a supermolecular spiral structure. Considering the chiral dopant molecule is located in the center of the "repeating unit", this cubic unit should be formed $10^{6}$ liquid crystal host molecues, meaning by 100 by 100 by 100 lattice in the $\mathrm{x}, \mathrm{y}, \mathrm{z}$ directions, respectively. The chiral dopant molecule interacts with liquid crystal molecules that are positioning 50 molecules away in each direction. As we known, the interaction between the chiral dopant and liquid crystal molecule is weak, mainly from the Van der Waals force between the molecules. It is hardly convincing that such small force could have such huge impact. There has to be path so that a chiral molecule can "pass" its chirality to the host molecule next to it. Then, this deracemistic host molecule could pass chirality to its neighbor. Then, pass again, finally reaching all the molecules in the host. The chirality passing path results in a long-range orientational interaction. This mechanism is also described and numerically simulated in literatures [53] [33]. 
2.5 Other methods Measuring Helical Twisting Power

\subsubsection{Reflective spectroscopic Method}

The reflectivitive spectroscopic method is normally used when substrates have planar alignment, and the helix of the cholesteric structure is parallel to the light propagation direction. In this case, when light incident into the liquid crystal cell, the circular polarized component with same handedness as the helix got reflected, and the circular polarized light that has the opposite handedness propagates. When

scanning the wavelength, $n_{-}^{2}$ is negative when $n_{-}^{2}$ is negative when $\lambda_{0}$ between $n_{-} p$ and $n_{+} p$. Thus, the pitch can be calculated by the spectroscopic method [39] [40] [41]

\subsubsection{Wedge Cell Method}

One of the most straightforward methods for the determination of $\mathrm{p}$ is called Grandjean-Cano wedge method [42] [43]. In a traditional wedge cell setup, the two 

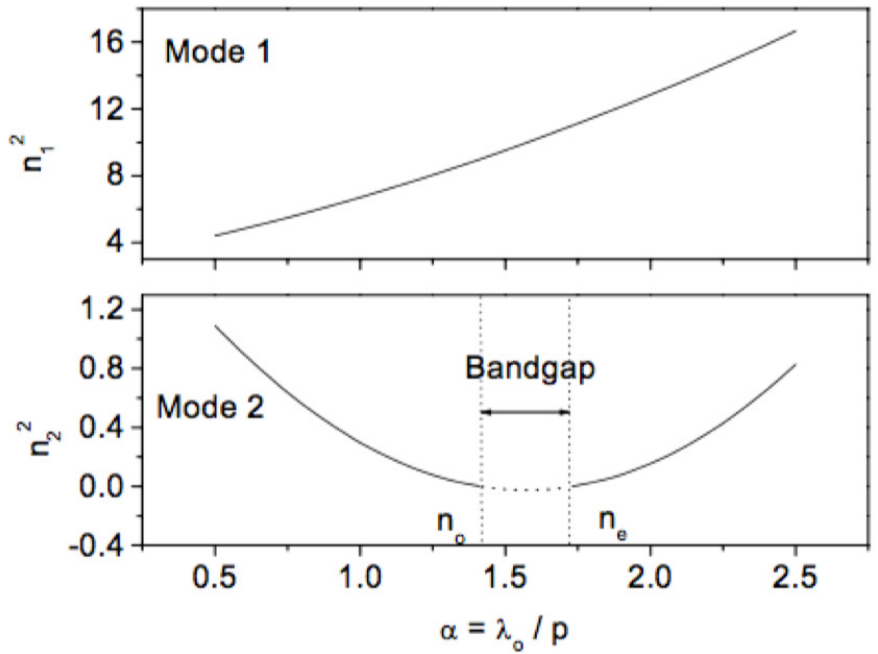

Figure 21: $n^{2}$ as a function of wavelength for two normal modes in bulk CLCs. The dotted line in mode 2 is negative. 


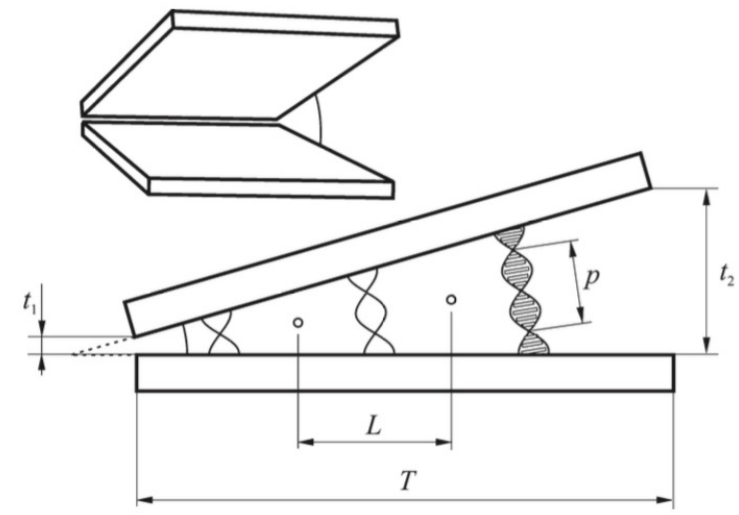

Figure 22: Setup of Grandjean-Cano Method.The incident light is monochromatic. 
substrates are prepared with anti-parallel alignment, where the alignment direction is perpendicular to the wedge. After capillary filling, the cell is heated to $100^{\circ} \mathrm{C}$ for $5 \mathrm{~min}$, then cool down to room temperature at the speed of $1^{\circ} \mathrm{C} / \mathrm{min}$. The disclination lines could be clearly seen through optical microscopy, as shown in Figure 23. Since the gap between each disclination is constant, by plotting the location and the number count of disclination lines, one could calculate the absolute pitch of cholesteric liquid crystal.

In this setup, the two substrates are assembled with spacers on a single edge, and the cell is made into a wedge shape as shown in Figure 22. The two substrates form angle $\alpha$. And surface alignment made on the substrates are anti-parallel, so helix structures are perpendicular to the substrates, and cell gap increases along the wedge. When the cell gap equals to the integer number of pitches, helix structure are formed. When the cell gap is between two integer number of pitches, disclination lines are formed because of the discontinuity of helix. The pitch can be calculated from distance $\mathrm{L}$ between the disclination lines and wedge angle $\alpha$

$$
p \approx 2 L \alpha
$$

The wedge cell method is easy and straight-forward; however, the hand-made wedge cells can induce errors, especially when determining the original and calculating 

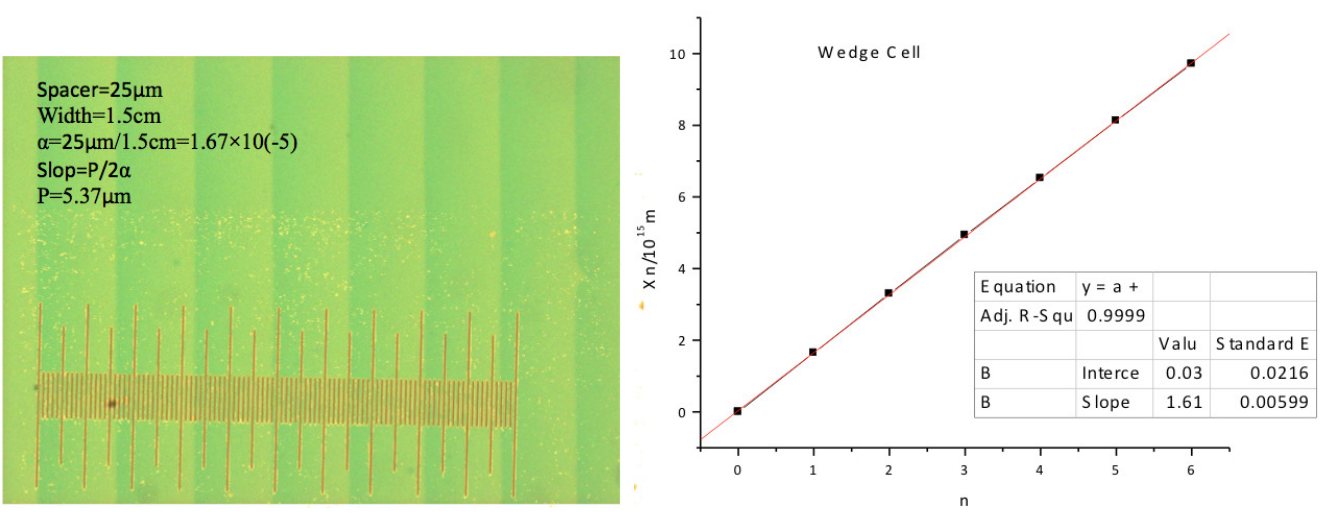

Figure 23: Left: liquid crystal disclination line observed through optical microscopy; right: distance to the wedge tip is linear with the number of disclination lines 

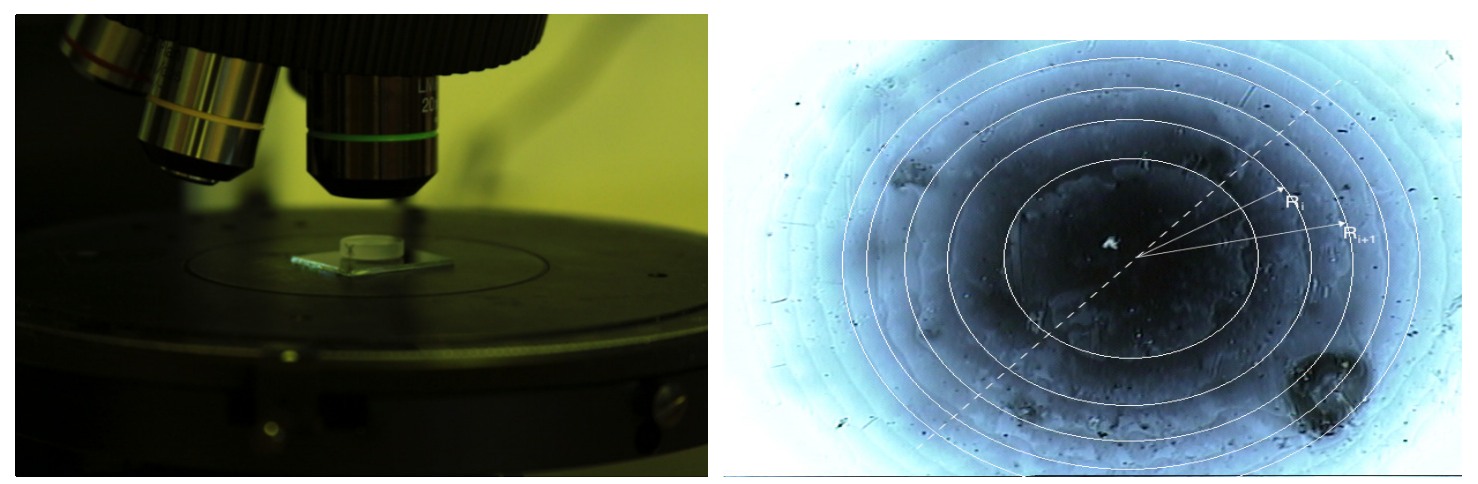

Figure 24: Disclination rings in advanced Crandjean-Dano method, using lens as one substrate, $2 \% \mathrm{CD} 6+98 \% \mathrm{E} 7$ between two crossed polarizers. Radius of Curvature $R=51.680 \mathrm{~mm}$, Newport.com 
the angle, $\alpha[54]$. And advanced method is to use a spherical lens with a well-defined radius as one of the substrates. A parallel aligned flat glass is used as the other substrate. Since the radius $r$ is known, or accurately measurable. By measuring the radius $R_{i}$ of each disclination ring as seen in Figure 24 , the pitch can be calculated as

$$
p=\left(R_{i+1}^{2}-R_{i}^{2}\right) / r_{l e n s}
$$

In this thesis, most of reported HTP is measured with lens setup, unless specially noted.

\subsubsection{Bragg Scattering Method}

Cholesteric liquid crystals are periodic optical media. Bragg scattering is also a popular method to determine the pitch of acholesteric liquid crystal [44]. During this test, a He-Ne laser is used to obtain monochromatic light. Cholesteric pitch can be determined by analyzing the angular dependence of scattered light [45].

\subsubsection{TN Cell Method}


(a)
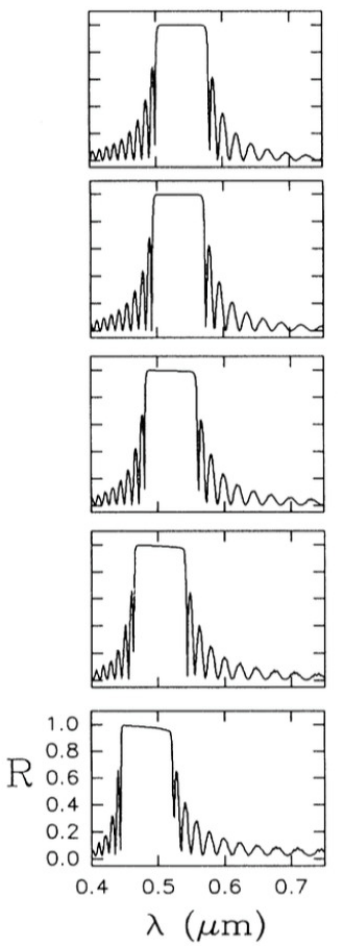

(b)
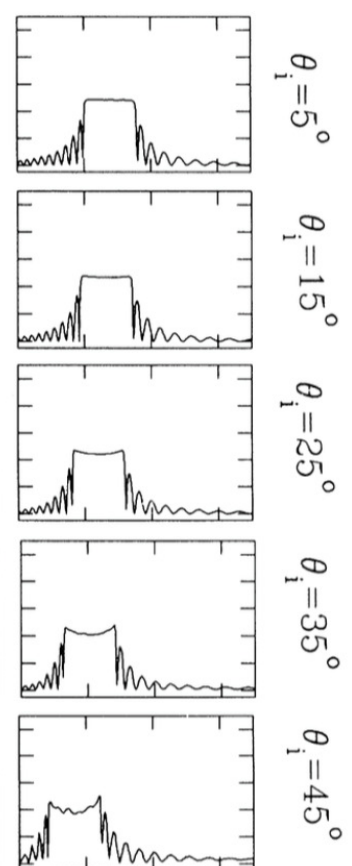

Figure 25: Theoretical reflection spectra for (a) incident right circular polarization and (b) unpolarized light at five angles of incidence. 


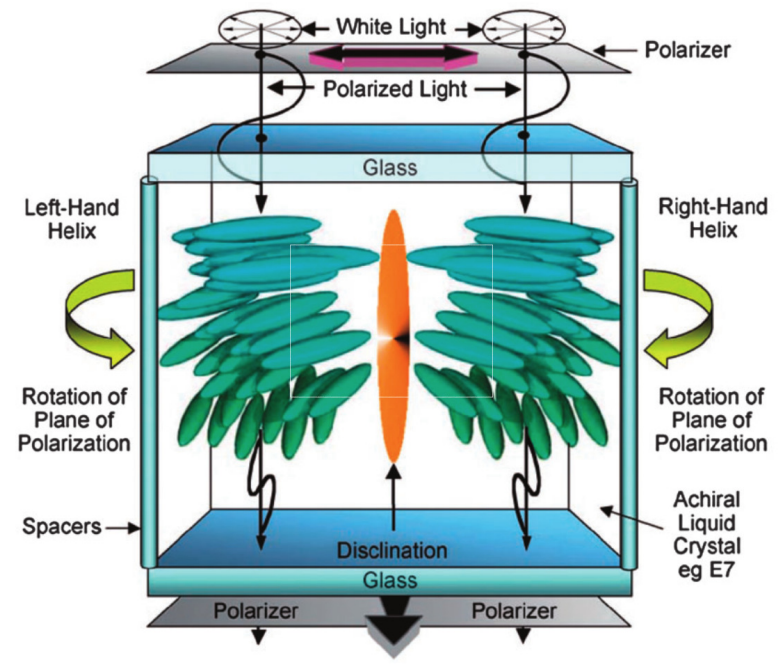

Figure 26: A twisted nematic cell showing degenerate left- and right-hand domains separated by a disclination line. 
TN cells are widely used in Liquid Crystal Displays, so the raw material of this method is commercially available. TN cell method is a newly developed technique for measuring large helical pitches with better accuracy and sensitivity. [46] [47] [48]. Unlike the conventional TN cell are uniformly aligned, the surface alignment for the test TN cells are aligned perpendicular to one another, as seen in Fig 26.

If the cell is filled in by an achiral or racemic liquid crystal, there is no preference for the liquid crystal to rotate left-handed or right-handed, hence, disinclination lines appear as straight lines. When filling with a chiral liquid crystal, the chiral bias breaks the degeneracy in the TN cell. One twist area is preferred over the other, the disinclination line moved towards the non-preferred area, so the line become curved. The more the chiral excess, the more curved of the disinclination lines, and the tighter the pitch. In Figure 27, it shows the same liquid crystal host a) with a racemic chiral additive, and b) with small amount of chiral excess, c) with large amount of chiral excess. If the radius of the disinclination lines can be measured as $R$, the related pitch $p$, can be written as,

$$
p=2 R
$$



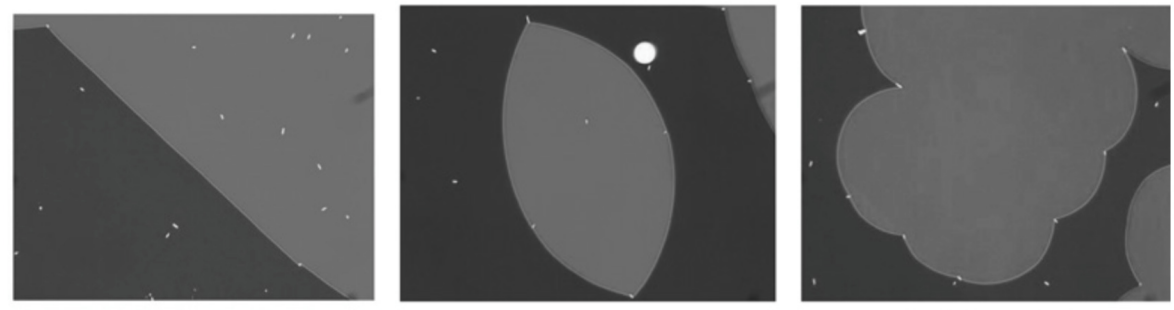

Figure 27: A twisted nematic cell showing degenerate left- and right-hand domains separated by a disclination line. 


\subsection{Conclusion}

In this chapter, we reviewed the chirality in liquid crystal. We covered the basic concepts of liquid crystal structure and mainly focused on cholesteric liquid crystals and chiral dopants. We also introduce the concept of helical twisting power (HTP), which is a measure of ability of a chiral additive to twist a liquid crystal into a helical structure. A list of common helical twisting power measurement methods are also discussed. 


\section{CHAPTER 3}

\section{Conformation and Chirality in Liquid Crystals}

In Chapter 1 and Chapter 2, we discussed the phenomenon of chirality and how chirality effects liquid crystals. Common sense dictates that once a chiral dopant or chiral molecules are added into achiral liquid crystal, the achiral liquid crystal will follow the chiral dopant and form a cholesteric structure. However, the cholesteric chirality is not necessarily to be exactly the same as the chirality of the chiral molecule. It is still unkown how chiral molecules interact with an achiral liquid crystal host and such small amount of additive turns bulk material into a chiral nemactic phase. In this Chapter, we will discuss the relationship between chiral dopant handedness and liquid crystal handedness.

\subsection{Introducing a Helical Structure into an Achiral Host}

It is known that the chiral liquid crystal phase can be introduced by dissolving a chiral dopant into an achiral liquid crystal host. Various materials can be used as chiral dopants in liquid crystals. 
The most common method is to add small molecules with molecular chirality into liquid crystals. A lot of fascinating works has been done by adding chiral molecules into achiral liquid crystal hosts. [55] In fact, most of the commercial cholesteric liquid crystals are designed by introducing a chiral core into the original liquid crystal molecular structure. In this way the chiral dopant can have chirality, and also easily to dissolve in liquid crystal bulk because of structure similarity with liquid crystal molecules. A series of popular chiral dopants, for instance, CB15, S811, and R811, are designed based on this concept.

Another series of compounds are commonly used as chiral dopant are molecules with chiral superstructures. One particular series of molecules are the bananashaped, also called bow-shaped molecules. This kind of molecules can form 3D helical supramolecular structures in a smectic C (SmC) phase [56] [57]. Jeong and coworkers demonstrated $B P C A C 6 P$ molecules forms head-to-head bended dimers by hydrogen bonds, then these dimers form superhelical structures as shown in Fig 28.

Interestingly, people also found that in certain circumstances, even non-chiral molecule can introduce a localized helical supramolecular structure [58] [59]. Another important experiment was also shown by Jeong and coworkers. In the experiment, 


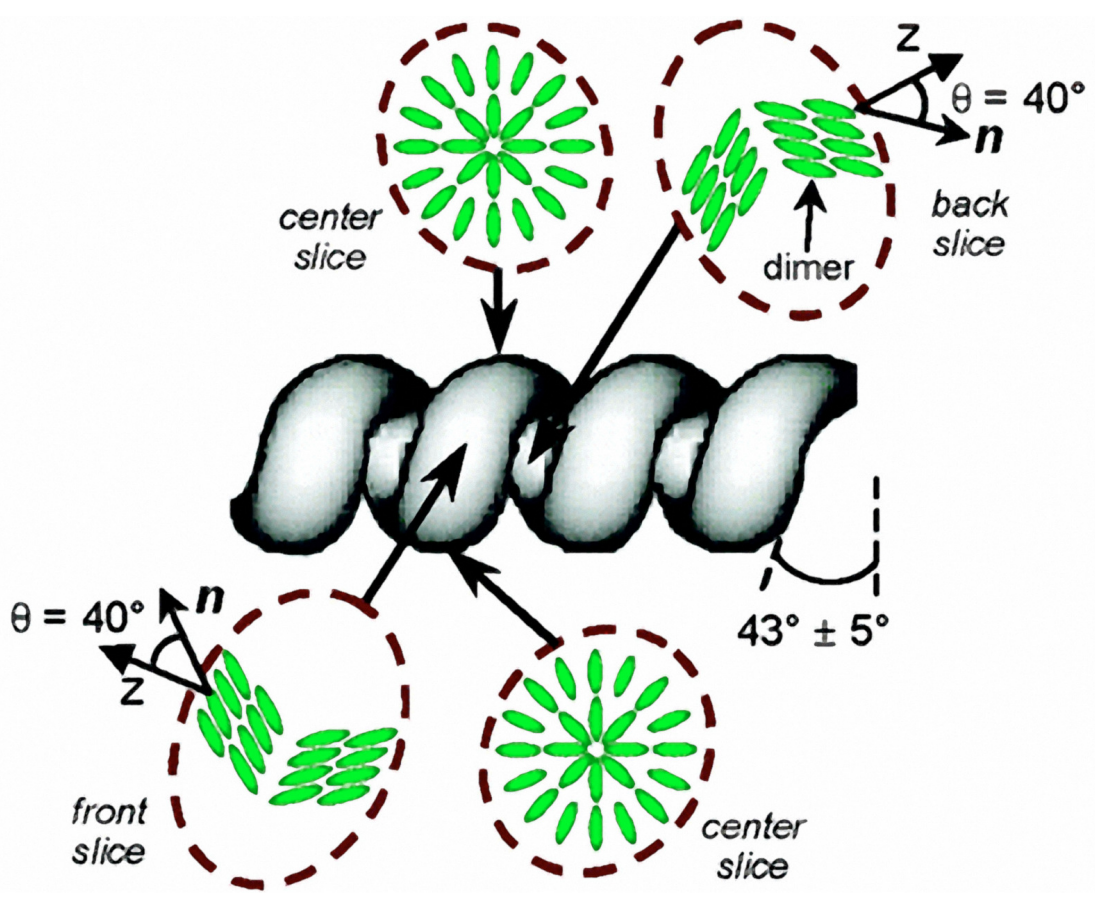

Figure 28: Schematic illustration of the BPCA-C6-P dimmer orientation in the helix 
$B P C A C n P m O H$ was dissolved into liquid crystals and heated up to the isotropic state. When the temperature cools down, droplets with different handedness were observed under polarized optical microscope, as seen in Fig 29a and b. Simulation proved that inside the droplets, the molecules form double twisting superstructure. At the center of the droplet, the molecular direction is perpendicular to the cell substrates, and at the edge, the director is parallel to the cell substates. The pitch of the helix is equal to the diameter of the droplet. Once the droplet with different handedness melt, the localized chriality disappears and racemic droplet appears. This experiment is a good example of chirality formed in achiral liquid crystal bulk.

Although chirality of liquid crystals can be introduced by various methods, using different chemical compounds, the general concepts have a lot of similarity. No matter if adding small chiral molecules to form a chiral liquid crystal bulk, or using chiral supramolecular structure to form chirality, or introducing chirality by adding chiral surface, all of the methods are not generating chirality, but passing chirality from the source, either a chiral molecule, or a chiral surface, to a bulk material. The chiral source can be a chiral molecule or a chiral surface, in the medium that chirality could be passed from one molecule to another, and finally reaches to every molecule in the bulk. Liquid crystals are very good medium for passing chirality. As is well known, all the molecular chirality comes from the structure of the molecule, and when 


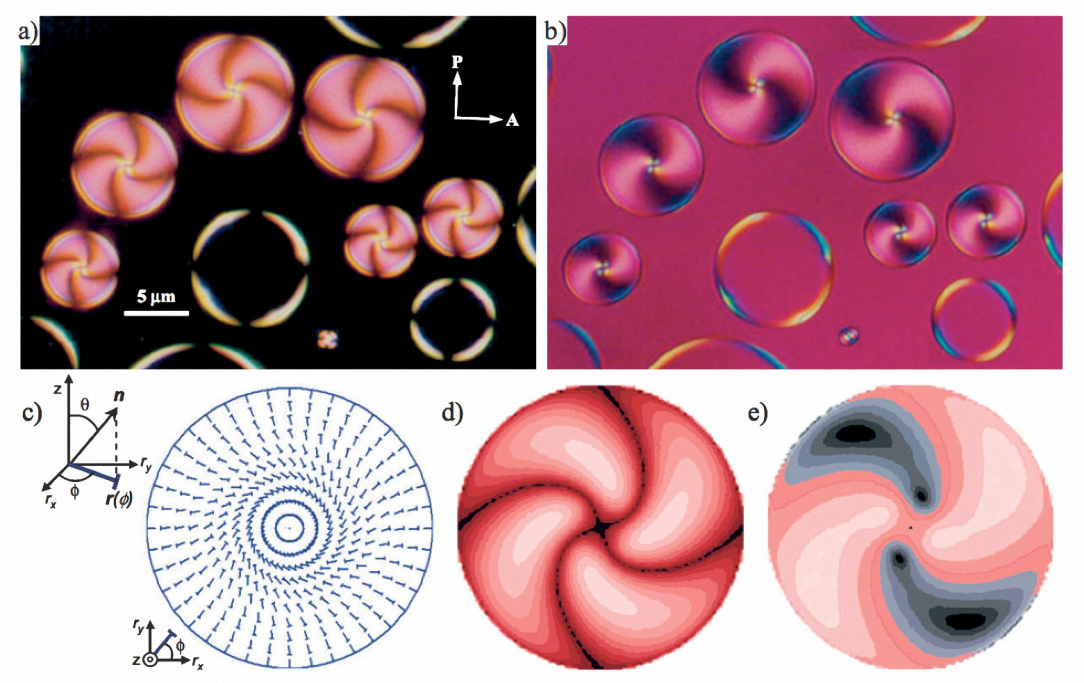

Figure 29: Propeller-patterned chiral N POM textures of BPCA-C7-PmOH in a bare glass cell: POM textures a) without and b) with a tint-retardation plate $(530 \mathrm{~nm})$ between the analyzer and the sample at 216 C. c) Computer-simulated molecularpacking model in a right-handed propeller-patterned $\mathrm{N}$ droplet. Here, $\mathrm{n}$ is the $\mathrm{LC}$ molecular director with the twist angle $(\theta)$ from the cylinder axis $(\mathrm{z}), \mathrm{r}(\phi)$ is the projection of $\mathrm{n}$ onto the $r_{x} r_{y}$ plane, and $\phi$ is the azimuthal twist angle. Calculated optical textures based on the molecular arrangement in (c) under POM d) without and e) with a tint-retardation plate between the analyzer and the sample. 
structure, or conformation changes, the chirality of molecule changes.

\subsection{Chiral Conformer}

As early as 1937, Kemp and coworkers found that when rotating a molecular substructure, like rotating the two $\mathrm{CH} 3$ groups around the $\mathrm{C}-\mathrm{C}$ single bound in ethane, a minimum energy could be found [60]. Base on entropy modeling, the lowest energy state could be found. It is a conformation with a dihedral angle of $60^{\circ}$, called the staggered state. The highest energy state is a conformation called the eclipsed state, in which the hydrogens at the forward carbon are directly in front of those on the back carbon. The energy barrier between the eclipsed state and staggered state is as low as $12 \mathrm{KJ} / \mathrm{mol}$. This means in ethane, the rotation around the Carbon-Carbon single bound happens very often even at a low temperature. In Fig 30, one can also tell that, in ethane, because of the symmetric structure, both of the carbons are connected only to hydrogens besides each other. The minimum energy status (staggered state) could be found at $60^{\circ}, 180^{\circ}, 300^{\circ}$ and these three staggered states are not distinguishable.

If one of the hydrogens on each carbon are substituted by another moiety, the symmetry is destroyed. For example, if one hydrogen on each carbon is replaced with 


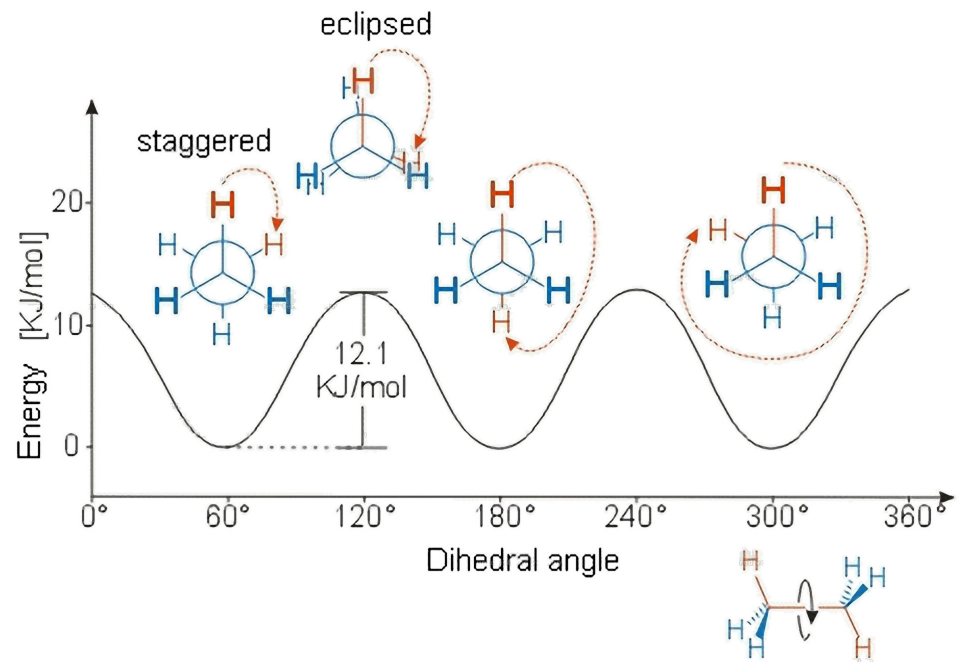

Figure 30: Ethane Barrier: Energy as a function of rotation around C-C bound 
a methyl group, we get the butane molecule. In butane, the staggered state spilt into two different groups: a dihedral angle $180^{\circ}$ status is the minimum energy state, called anti staggered; and $60^{\circ}$ and $300^{\circ}$ cases form multi-minimum status, called gauche states. The same as staggered states, the eclipsed state also split into two: the fully eclipsed highest energy state and the partial eclipsed state, as seen in Fig 31 . The two partial eclipsed states and the two gauche states are two pairs of enantiomers. As one can see, the more complicated the structure is, the more conformations can be formed, and even achiral molecules can form chiral conformers under certain circumstances.

Another class of compounds that display conformational chirality are biphenyl and its derivatives. As shown in Fig 32, the two benzene rings in biphenyl are not in the same plane, one is slightly twisted relative to the other due to steric crowding. As a result, an almost $45^{\circ}$ dihedral angle is formed between the two benzene rings and the center $\mathrm{C}-\mathrm{C}$ single bound [61]. Note that in the biphenyl case, the dihedral angle can be positive or negative, there are two enantiomers with opposite handedness. Only when the dihedral angle equals $n \pi / 2$, meaning when the two phenyl rings are parallel or perpendicular to each other is the molecule non-chiral; all the other dihedral angles make the molecule non-superimposable with its mirror image, which makes the molecule chiral. 


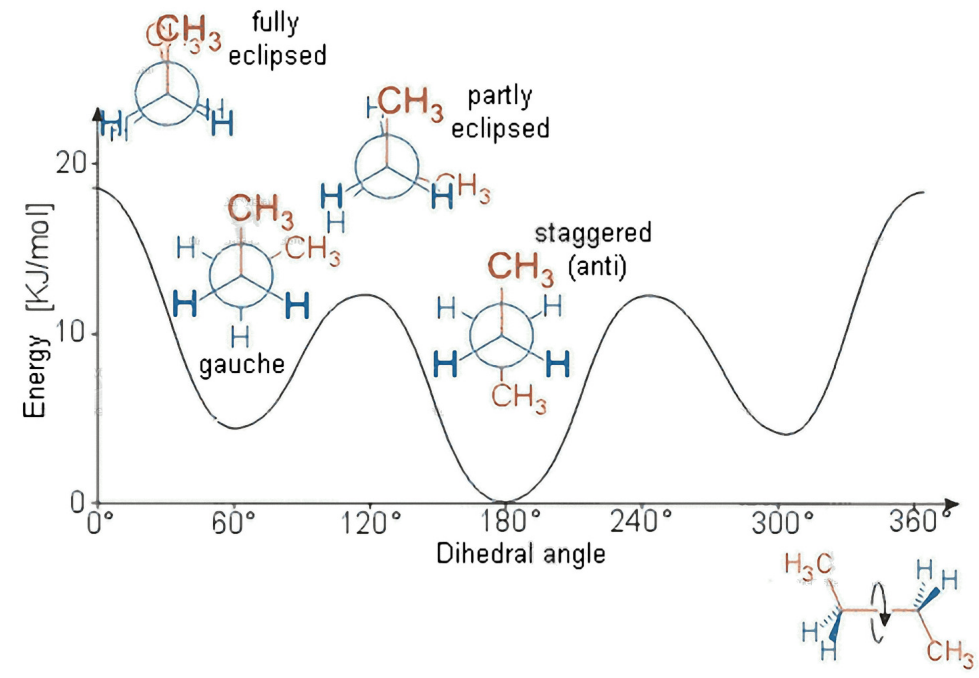

Figure 31: Energy as a function of rotation around center C-C bound in butane 


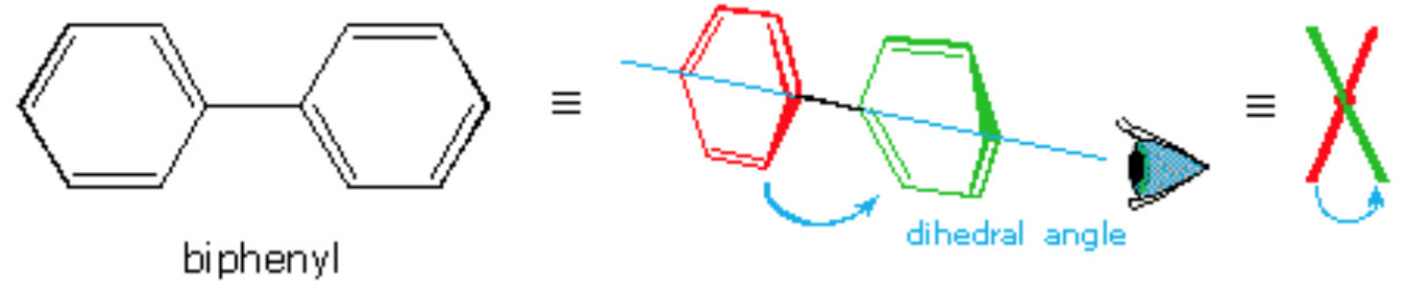

Figure 32: Energy as a function of rotation around center C-C bound in butane 
When the positions next to center C-C $(2,6)$ are filled by hydrogens, small energy barriers can hardly stop biphenyl from rotation [62] [63], see Fig 33. In fact, at room temperature, the two benzene rings are rotating along center $\mathrm{C}-\mathrm{C}$ single bound rapidly. However, when the substituents are bigger groups, like $-\mathrm{COOH}$ or $-\mathrm{SO}_{3} \mathrm{H}$, once the twist angle is formed, it becomes harder for the benzene rings to rotate from one side to the other at low temperature. Conventionally, an activation energy barrier of 16 to $19 \mathrm{kcal} / \mathrm{mol}$ is necessary to prevent spontaneous room temperature racemization of substituted biphenyls [64] [65].

Actually, most liquid crystal molecules have this kind of biaryl core structure. In order to increase the solubility of the chiral compounds and liquid crystals, this kind of chiral compounds with an unresolvable biphenyl-core from a series of chiral dopants. A simple stereochemical model [66] [67] explains this interaction. As both of nematic host and chiral guest have a biaryl structure, typically, the chiral guest has a well-defined and stable molecular helicity, and the liquid crystal host molecules are flexible. In a non-chiral environment, liquid crystal molecules can exist as either of the enantiomers, which behaves with opposite helicity. Furthermore, the enantiomers can rapidly switch between each other, and reach a chemistry equilibrium state that is non-chiral. When a chiral guest is doped into this system, the equilibrium state 
is changed, instead of equal number of enantomers, the host enantiomer with the same helicity as the chiral guest is preferred and obtains a lower energy state. More enantiomers switch from the opposite helicity to this helicity, and fewer enantiomers switch back, so the total liquid crystal host exhibits helicity the same as the chiral dopant helicity.

Typical cases of Energy vs diheral angle plots are listed in Table 3 and Table 4 shows plots of energy vs diheral angle that are simulated with Hyperchem. The bright blue line intersections represent to carbon atoms, and white ones represent a proton; and red ones represent oxygen. The calculated dihedral angle in each molecule is marked in green color.

Using the same concept, we can find the optimum dihedral angle for particular molecules. Table 5 illustrates a set of the small molecules all derivative from biphenyl tetrol. The only differences are the ring sized formed by the part of the two phenyl rings, the two phenol C-O bounds and the carbon in between the two oxygen groups. The number of the ring numbers varies from 7 to 11 . And the optimum dihedral angle is modeled by searching the lowest energy state using a conformational search. These modeling results show that the non-planarity changes when the ring member varies. 
Table 3: Table of energy vs dihedal angle -1

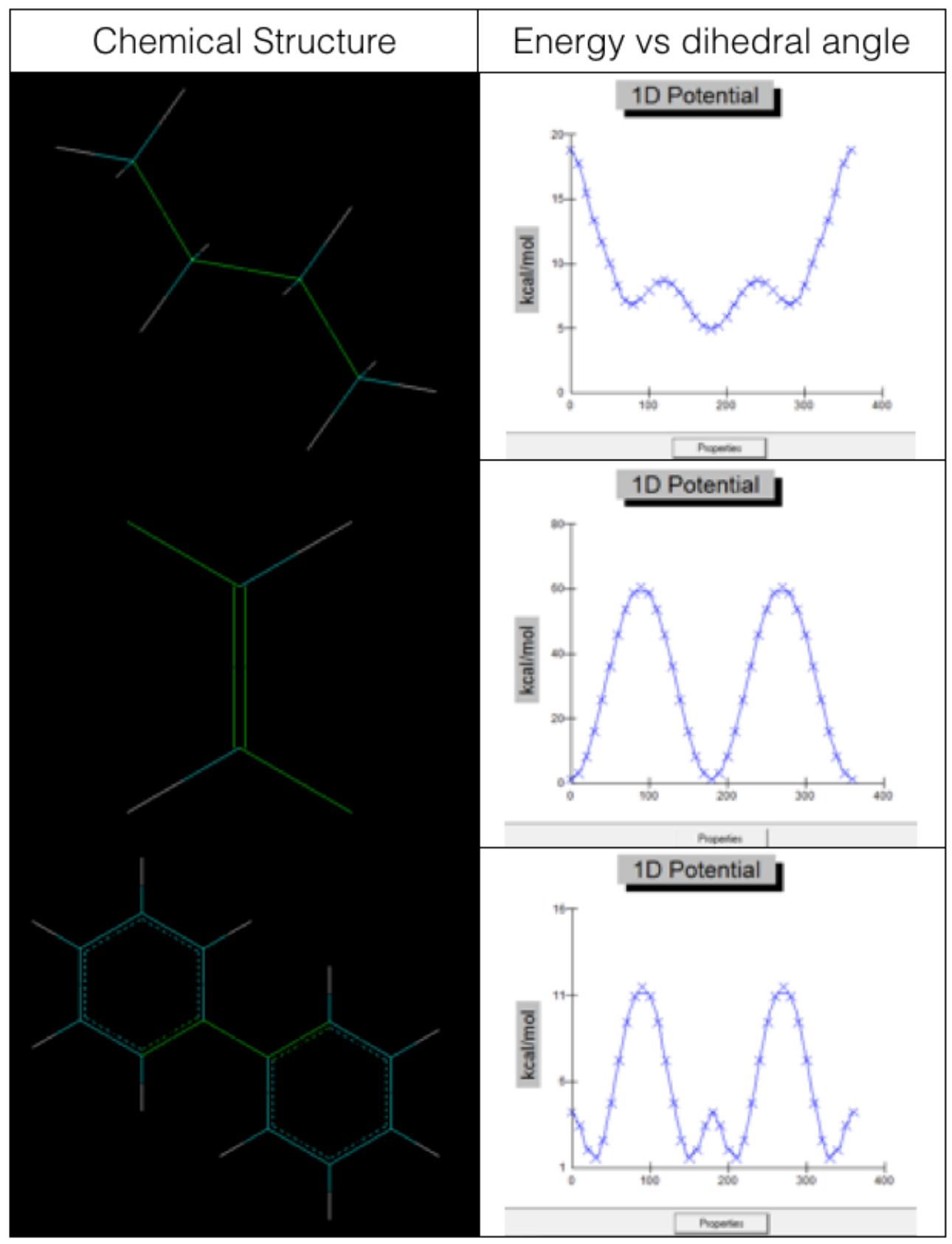


Table 4: Table of energy vs dihedal angle -2

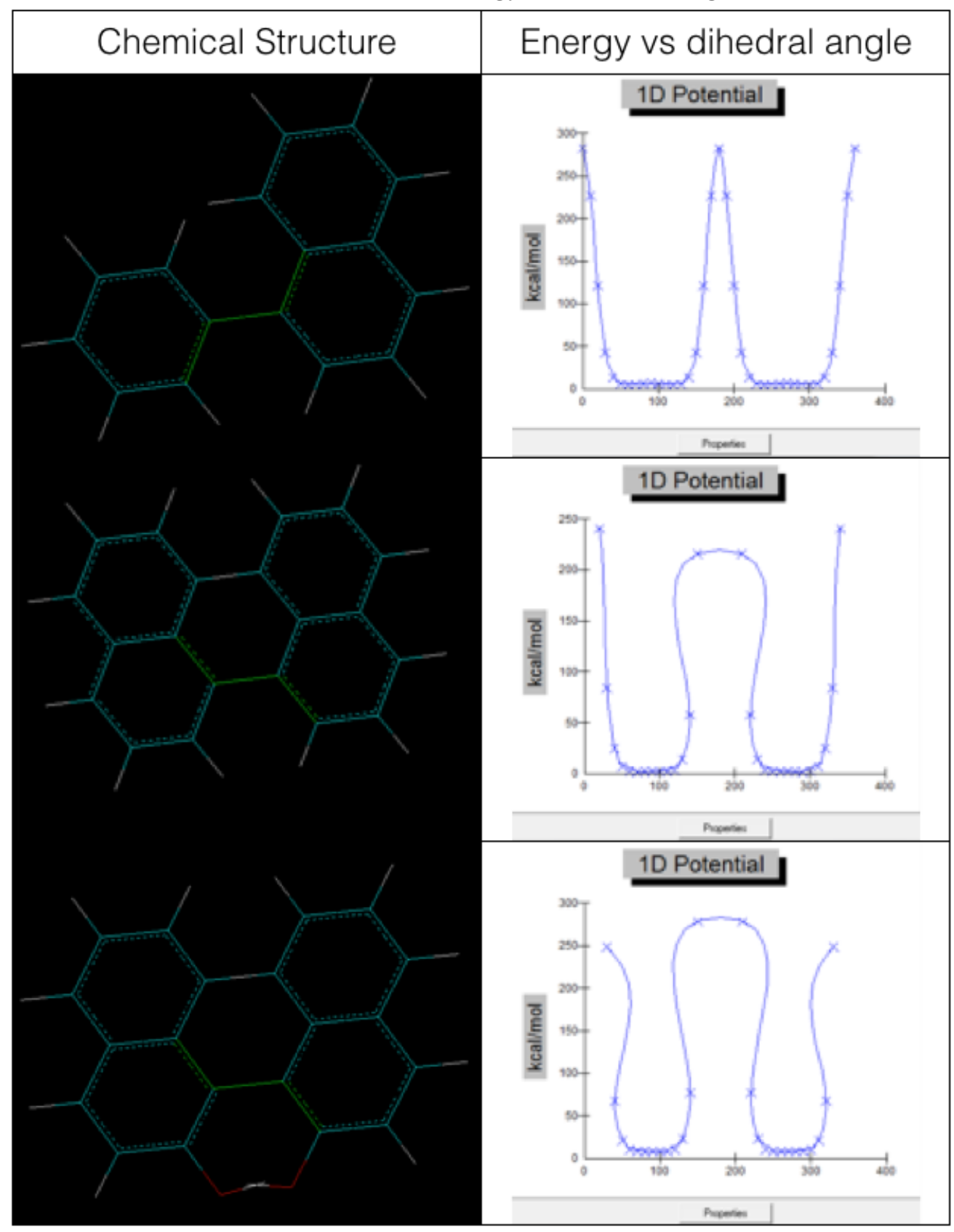


Table 5: Table of ring non-planarity modeling

\begin{tabular}{|c|c|c|}
\hline Sample & Chemical Structure & Dihedral Angle b/t Phenyl Rings \\
\hline $7 \mathrm{MR}$ & & $43.4^{\circ}$ \\
\hline $8 \mathrm{MR}$ & & $51.8^{\circ}$ \\
\hline 9MR & & $58.5^{\circ}$ \\
\hline 10MR & & $53.6^{\circ}$ \\
\hline $11 \mathrm{MR}$ & & $58.3^{\circ}$ \\
\hline
\end{tabular}




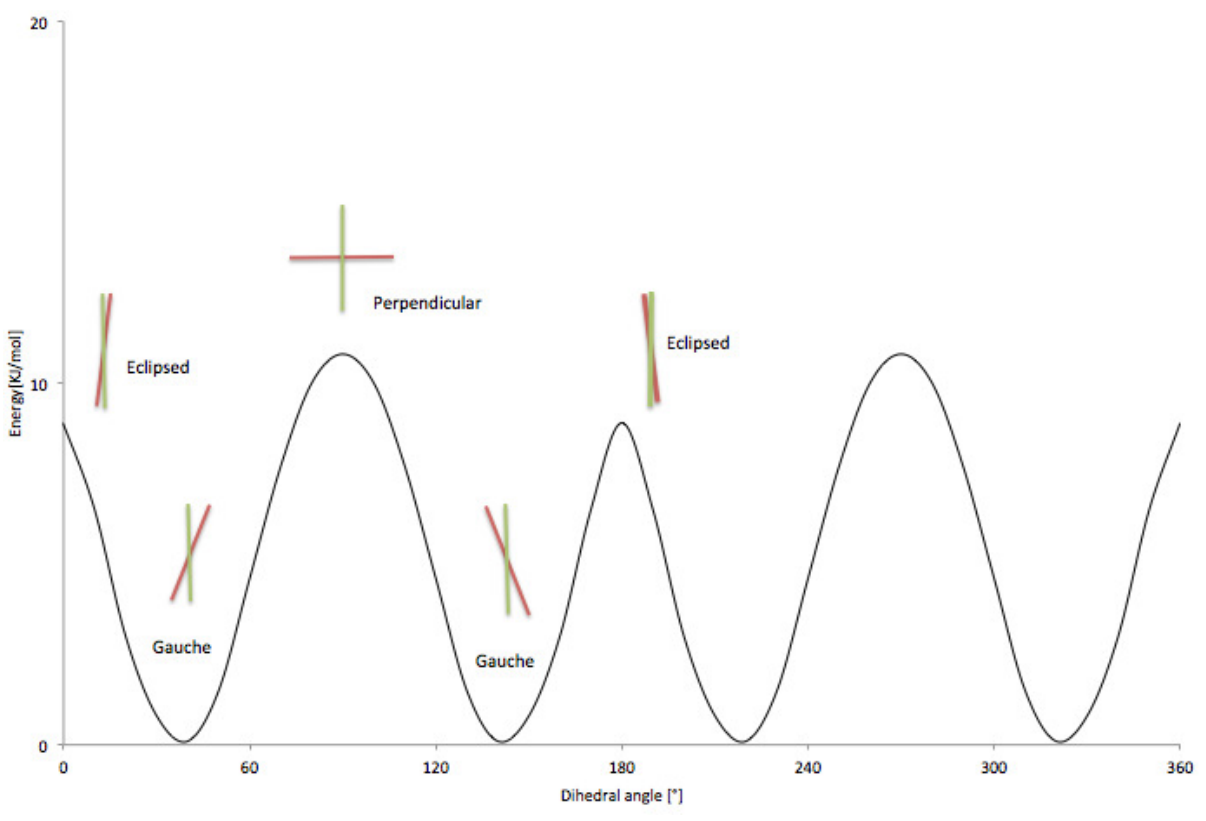

Figure 33: Energy as a function of rotation around center C-C bound in biphenyl 
When ploting dihedral angle vs the number of the C-O ring members, we can see when the ring size is small, geometric limitations play a big role in preventing the two phenyl rings from rotating out of the plane, and the dihedral angle is only around $40^{\circ}$, when there is only single carbon between the two oxygens. When the ring size gets bigger, the pulling effect of the ring gets much smaller, and the dihedral angle between the phenyl rings gets bigger. And finally, when the number of carbon atoms between the two oxygens reaches 3 , the pulling effect is completely gone, and the dihedral angle between the two phenyl rings is approaching to $60^{\circ}$, the dihedral angle for opened ring case in 2,2',6,6'-Biphenyltetrol.

\subsection{Conformation and Chirality}

Let us think about cyclohexane, molecular formula $C_{6} H_{12}$. It is an alkane with a six-membered ring structure. Unlike benzene, it does not conform to a shape of a perfect hexagon in a 2-dimentional plane, instead, it escapes to 3rd dimention and forms three-dimensional structures. They were originally named symmetrical and unsymmetrial structures by Hermann Sachse, when firstly discovered in 1890 [68]. [69]. [70]. They are now called chair and boat conformers. Fig 35 shows the chair and boat conformers for cyclohexane. 


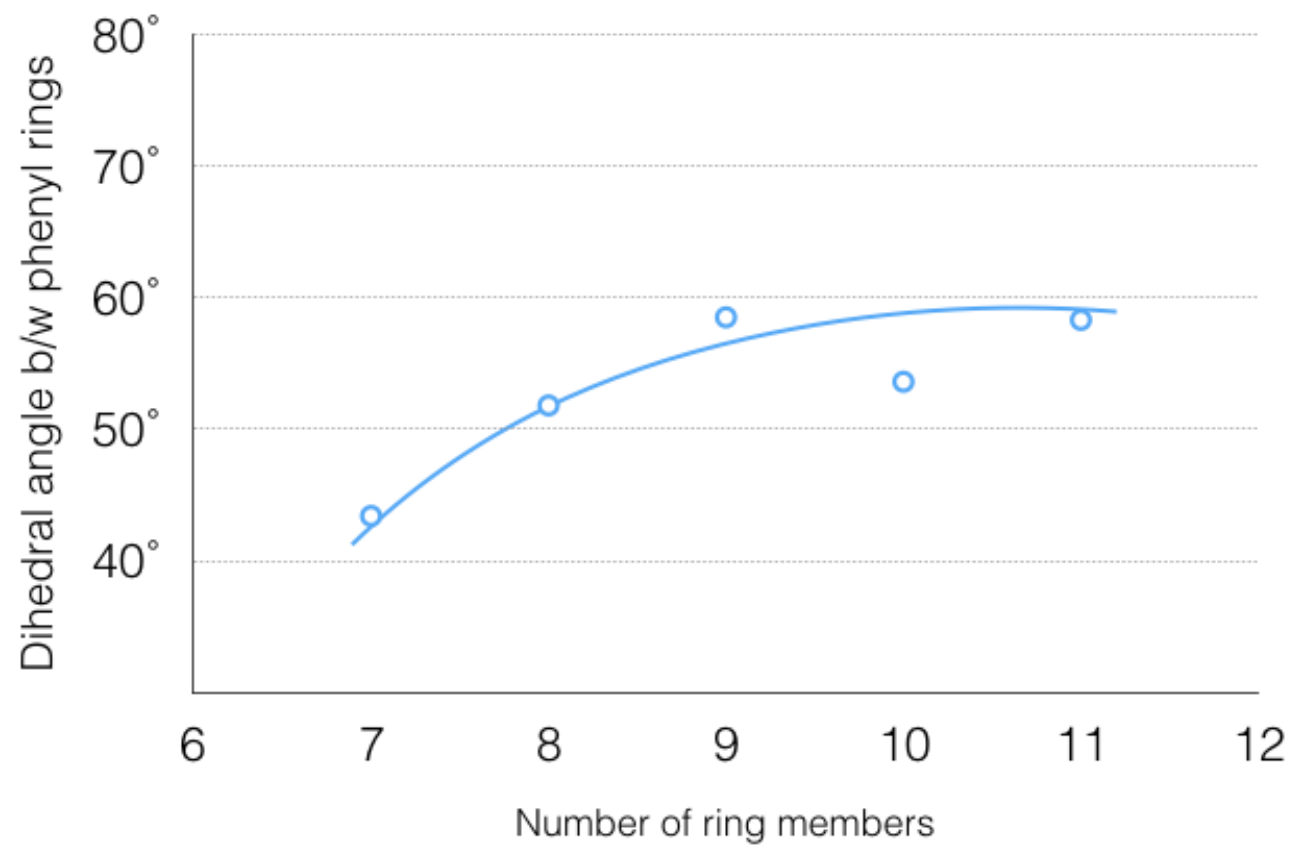

Figure 34: Dihedral angle between phenyl rings vs number of ring members 
Using HyperChem molecular dymanic modeling, we found that the energy of the boat state is $11.9 \mathrm{kcal} / \mathrm{mol}$, almost twice as much as the chair state of $6.55 \mathrm{kcal} / \mathrm{mol}$. In fact, between the two modes, there are two twist chair states exists. Although both of the twist chair states are chiral, both the boat and chair conformers are nonchairl conformers. But, when the hexane has functional groups attached to it, both conformers could become chiral comformers.

Applying the same concept, we modeled the isosorbide core. Interestingly, two lowest energy states showed up: one, which we called the boat-like core, shows the lowest energy state, $16.73 \mathrm{kcal} / \mathrm{mol}$; the other one, which we called chair-like core, shows a slightly higher energy state, $17.08 \mathrm{kcal} / \mathrm{mol}$. Fig 36 shows the boat-like core conformer in the top drawing and chair-like core conformer at the bottom.

When comparing the ethane, cyclohexane and isosorbide cores, when the structures get more complex, the possibility of for all the chemical bonds getting to the lowest energy status are getting smaller. In most of the cases, if this bond reaches the lowest energy state, the other one might be in a higher energy state due to geometric limitations. Therefore, the lowest state energy is larger, an energy difference between states gets smaller. In most of the cases, in isosorbide, we have a mixture of different conformers, which contains both chair-like cores and boat-like cores. 


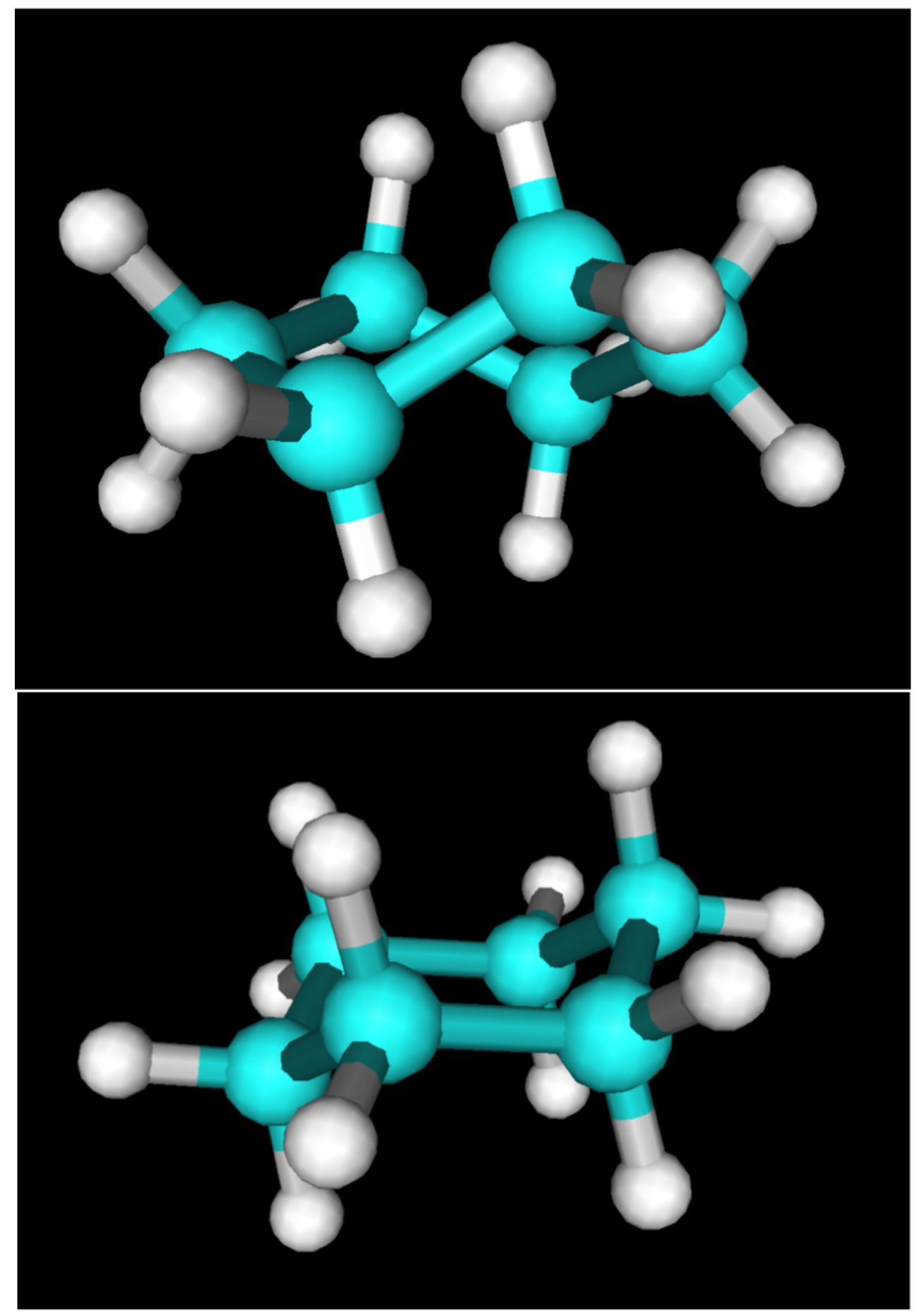

Figure 35: Boat (top) and chair (bottom) conformers for chclohexane 
We then added more complex carbon chains into the isosorbide core structure. Using CD6 as an example, the model shows two different conformers, with a chair-like core and a boat-like core, as shown in Figure 37. One formed a big spiral structure, the other one only has a small twist. The 5-membered-ring at the core of CD6 exist in two conformations termed the twist and envelope structure. The two rings of the isosorbide core exist in two predominant conformations termed the boat and chair, analogous to the structure of cyclohexane. Molecular modeling of CD6 shows that the boat and chair conformations are very different with the boat conformation taking on a more folded, spherical overall shape and the chair conformation having a more propeller like shape. These large changes in overall structure would be expected to produce distinctly different HTPs. Figure 37 shows the molecular models of these two conformations of CD6. The boat-like core CD6 shows a ball shape; while the chair-like core shows a propeller shape, which can induce more chirality. The molecular model predicts that the boat and chair conformers of CD 6 vary in free energy by about $3.5 \mathrm{kcal} /$ mole in vacuum. The exact conformation of the chiral additive will depend on the liquid crystal host and will differ somewhat from the model. 

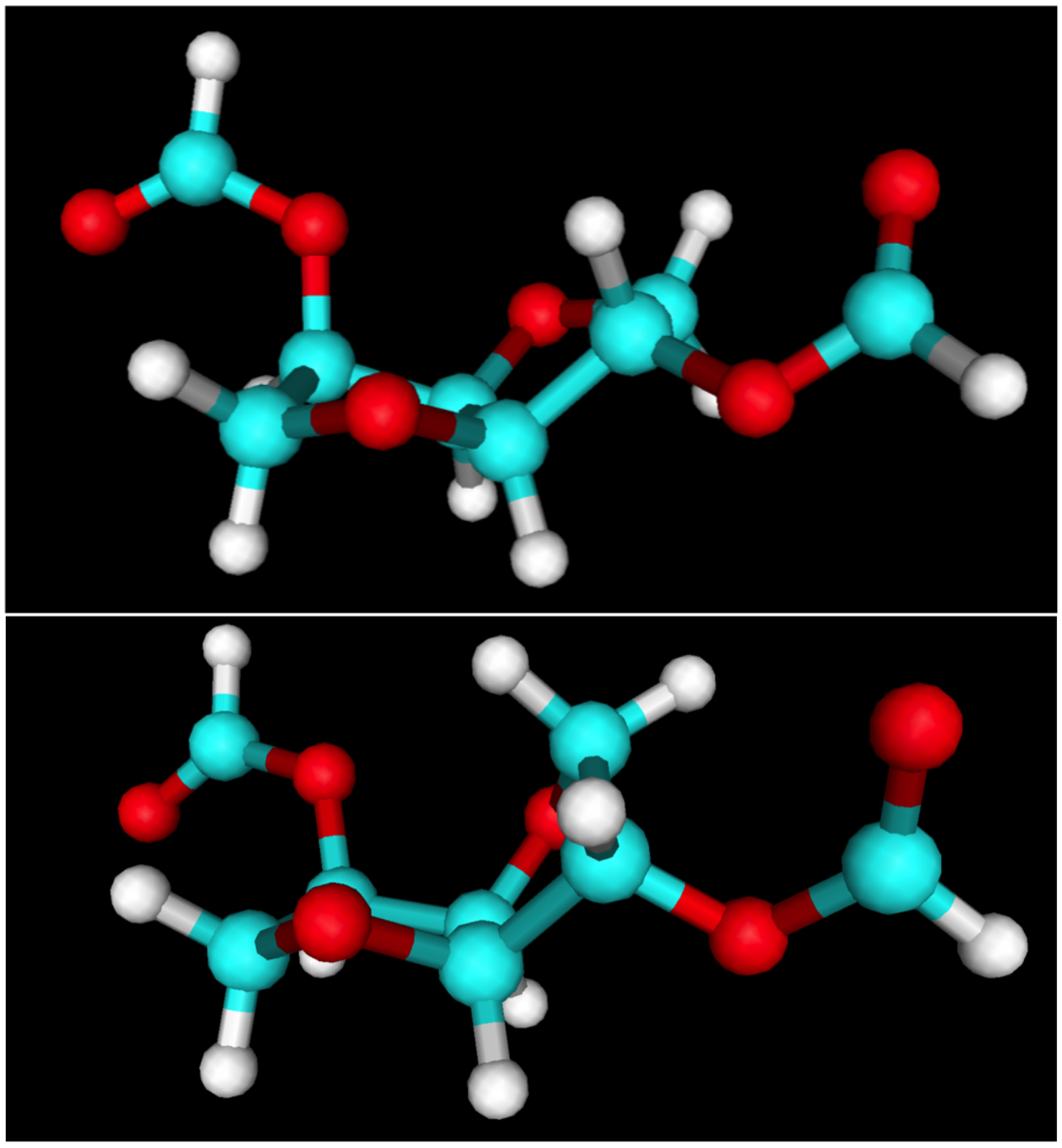

Figure 36: Boat-like (top) and chair-like(bottom) conformers for isosorbide core 


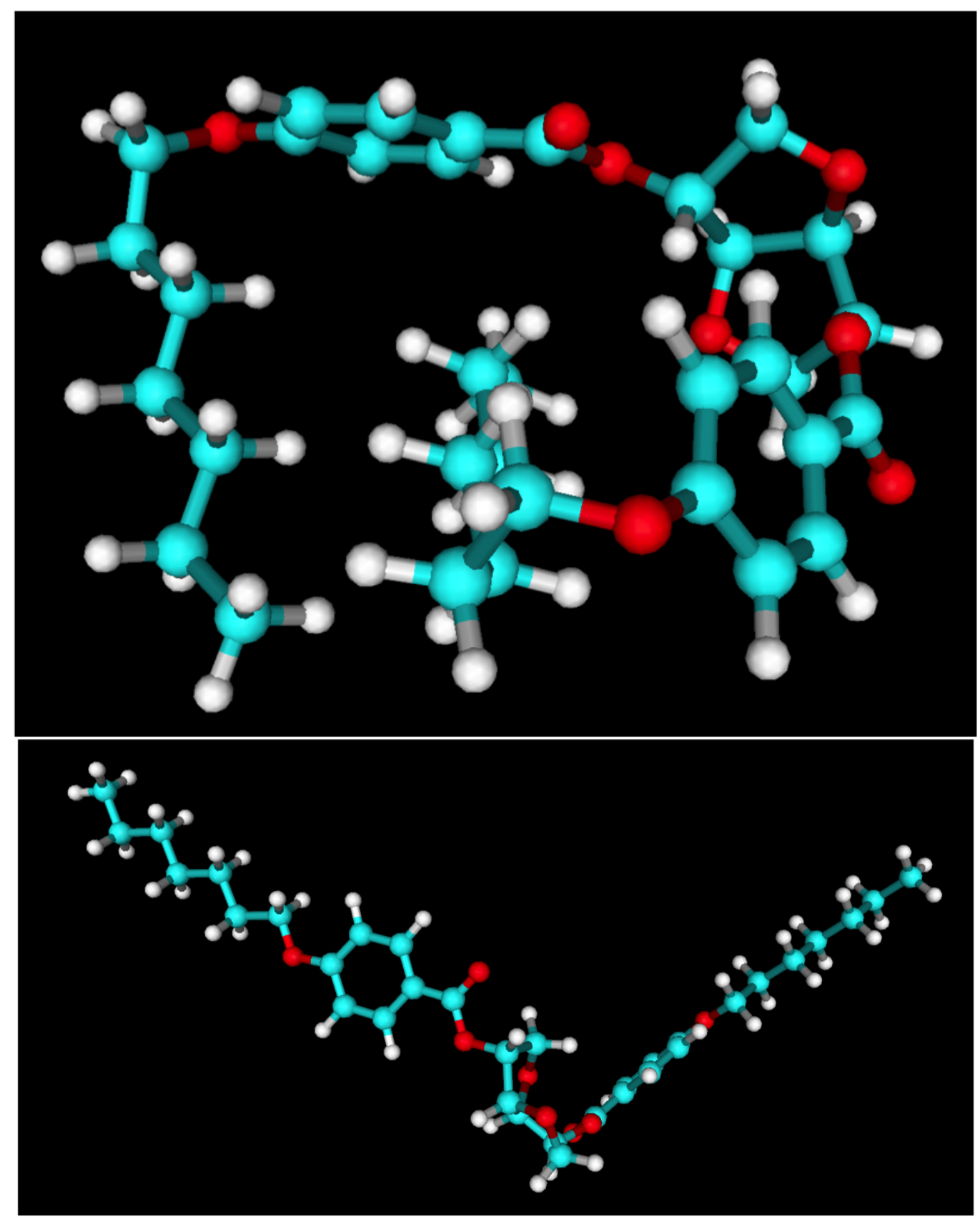

Figure 37: CD6 with boat-like core(top) and chair-like core(bottom) 


\subsection{Conclusion}

In this chapter, we started a discussion of how conformation variation induces molecular chirality. Using a molecular dynamics simulation tool, we are able to model molecular conformation and free energy for each state. By modeling the CD6 chiral dopant, we found two predominant conformers that show very different helical structure. The modeling results predict a big helical twisting power difference in different conformers. The difference will be experimentally examined in the next chapter. 


\section{CHAPTER 4}

Mechanism of Temperature Dependence of Pitch in High Helical Twisting Dopants

In Chapter 2, we explained that high helical twisting power chiral additives are required for an expanding the variety of liquid crystal displays and devices. Molecular conformation plays a critical role in determining the helical twisting power, HTP, of chiral additives. In this chapter, results of studies of additives based on an isosorbide benzoate ester core are presented. Molecular modeling in Chapter 3 revealed two low energy states with very different conformations for this core. Ultra-violet absorption and NMR spectra will show two stable isosorbide conformers. These spectra reveal how the relative populations of these two conformations change with temperature and how this is related to the helical twisting power. Conformation population changes can explain many of the observed anomalous responses of HPT to temperature. 


\subsection{Introduction}

Chiral liquid crystals have intrigued scientists since their first discovery in 1888. In particular, liquid crystals magnify the optical rotary power of a chiral material by orders of magnitude. They transfer chirality from the molecular level into a microscopic, periodic twisting of the liquid crystal director. The chiral nematic phase efficiently reflects light of a wavelength that is on the order of the period of the microscopic twist. When this twist is on the order of the wavelength of visible light, the material is brightly colored.

The mechanism of chiral induction in a nematic liquid crystal is of great interest both from a basic science perspective and for a variety of applications, including bistable cholesteric and blue phase displays. A recent review by Pieraccini and coworkers does an excellent job of summarizing the current understanding to chiral induction. [71]. The chirality of molecules is often described by their specific rotation of polarized light [72]. The specific rotation of organic molecules is temperature dependent. [73] This change is ascribed to changes in the distribution of molecular conformations and the resulting impact on the ability of a chiral center to rotate the polarization of incident light. This temperature dependence is small compared 
with the temperature dependence of the HTP for the materials dissolved in a liquid crystal host. What is important in respect to this chapter is the ability of specific chiral additives to induce helical twist in a nematic liquid crystal .

In general the ability of a chiral additive to induce a helical twist in the nematic phase is quantified by the helical twisting power, HTP. Materials with high HTP typically have two or more planar groups that are twisted relative to one another inducing structural chirality. While the HTP is enhanced with relatively rigid molecular structures [67], solubility requires some conformational flexibility. The HTP varies for different conformers and the measured HTP will be a weighted average of the ensemble of conformers.

The HTP of a chiral additive often varies as a function of temperature. Interestingly, the HTP can increase, decrease or remain relatively constant with temperature depending on the specific chiral additive and also on the liquid crystal solvent. This temperature dependence of the HTP of chiral additives has been effectively used to produce thermometers and other temperature sensing devices. However, most display applications of chiral nematics require little or no change in HTP with temperature. It is therefore important to understand the molecular basis for the temperature dependence of HTP and to design chiral additives accordingly. 
The dependence of helical twisting power on the conformational distribution of the chiral additive was used by Chilaya to help explain the wide variations in the temperature dependence of the helical twisting power for various chiral additive/liquid crystal solvent combinations. [74] Specifically, he noted that the relative population of the different conformations will depend on the free energy difference between the two conformations and the temperature based on the Boltzmann distribution. Slaney and coworkers discussed how this change in conformational distribution could be used to explain the sign inversion of the HTP of some benzoate esters [75].

\subsection{Experimental Details}

In this study, experiments were done to verify the relationship of the temperature dependence of HTP with changes in the conformational distribution of the chiral additive. A series of isosorbide derivative are studied. The isosorbide core structure is shown in Figure 38. And the list of the chiral dopant is listed in Table 6 . 


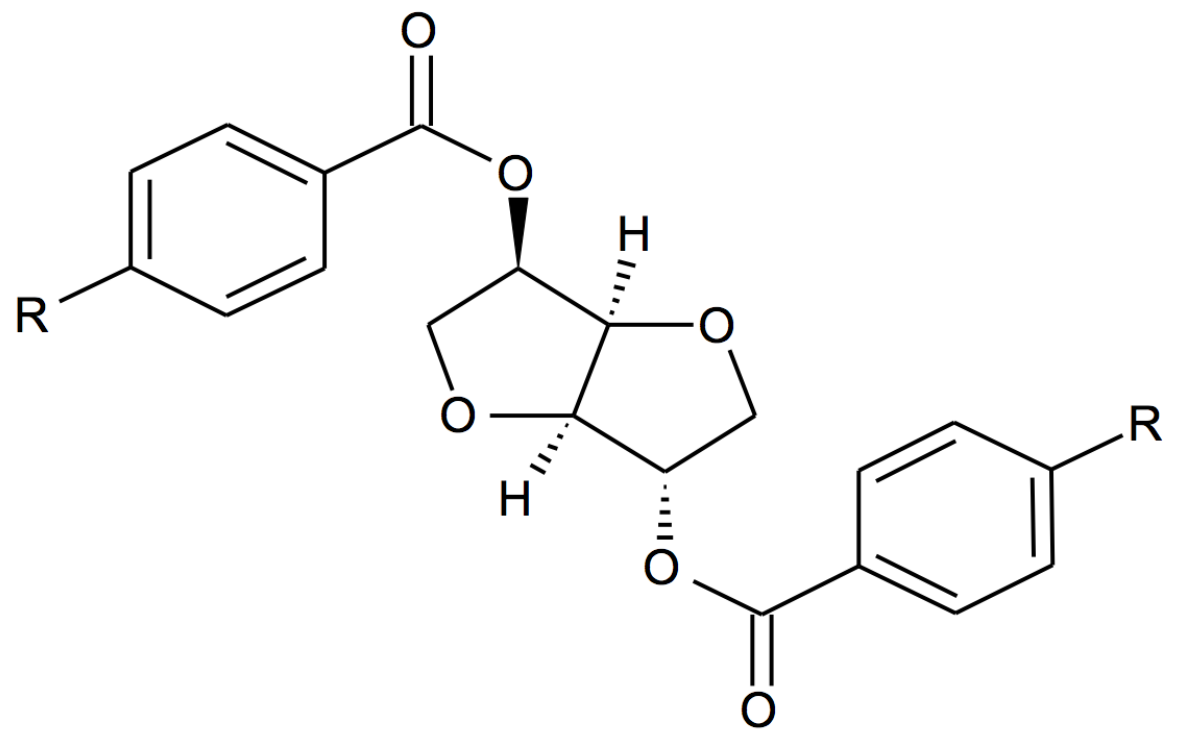

Figure 38: Molecular Structure of the isosorbide benzoate ester studied 
Table 6: List of chiral dopants materials

\begin{tabular}{|c|c|}
\hline CD\# & $\mathrm{R}$ \\
\hline 3 & $\mathrm{CH}_{3} \mathrm{O}$ \\
\hline 4 & $\mathrm{C}_{5} \mathrm{H}_{11} \mathrm{O}$ \\
\hline 5 & $\mathrm{C}_{6} \mathrm{H}_{13} \mathrm{O}$ \\
\hline 6 & $\mathrm{C}_{7} \mathrm{H}_{15} \mathrm{O}$ \\
\hline 7 & $\mathrm{C}_{12} \mathrm{H}_{25} \mathrm{O}$ \\
\hline 9 & $\mathrm{p}-\mathrm{CH}_{3} \mathrm{OPhCO}_{2}-$ \\
\hline
\end{tabular}




\subsubsection{Synthesis}

The chiral additive, an isosorbide benzoate ester, was synthesized by coworker, Dr Donald Diehl, purified and the helical HTP was measured as a function of temperature. The synthesis method is shown in Figure 39. [76] [77]. In the synthesis description, CD9 is used as an example.

\subsubsection{UV-Vis Spectroscopy}

Ultraviolet absorption spectra were measured using a Perkin Elmer, Lambda 9. Dilute solutions (adjusted to have absorption maxima around 1) of the chiral additive dissolved in heptane or octane were placed in a $1 \mathrm{~cm}$ quartz cuvette with a matched cuvette filled with heptane or octane used as reference. Spectra were measured between room temperature and $50^{\circ} \mathrm{C}$ for both heating and cooling cycles.

In order to study the chiral dopants conformational change with temperature change, the first experiment was done with chiral dopants dissolved in hexanes. Due 


\section{Method A. Acid Chloride Condensation}

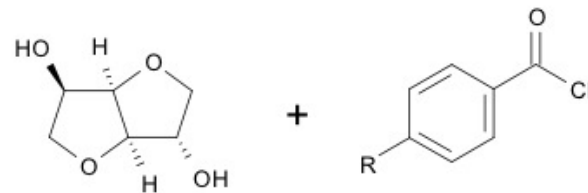

Dianhydro-D-glucitol

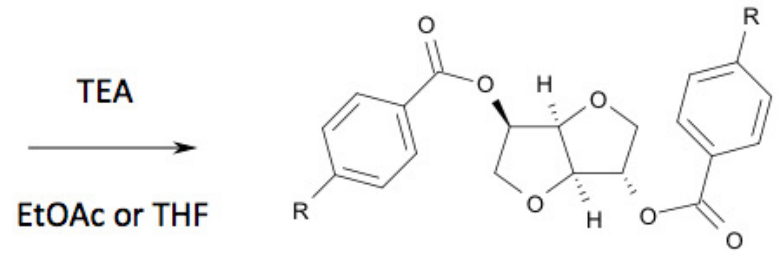

Isosorbide Chiral Dopant

Method B. Dicyclohexyl carbodiimide Reaction<smiles>O[C@@H]1CO[C@@H]2COC[C@H]12</smiles>

Dianhydro-D-glucitol

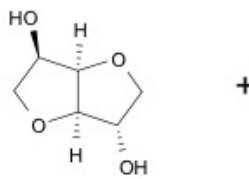<smiles>[R]c1ccc(C(=O)O)cc1</smiles>

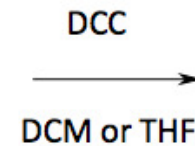
cat. DMAP

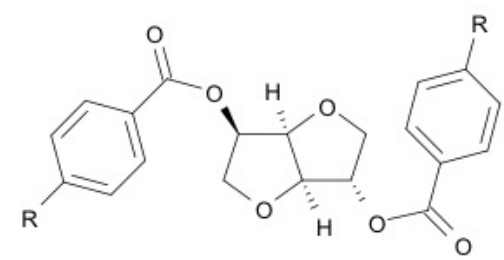

Isosorbide Chiral Dopant

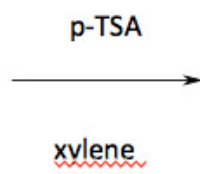

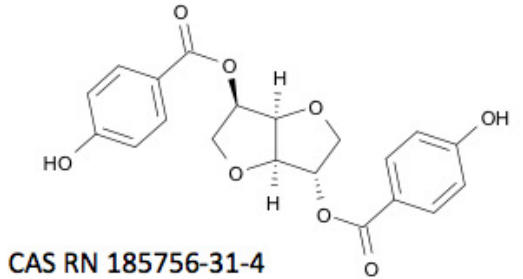

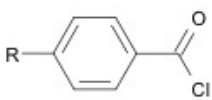

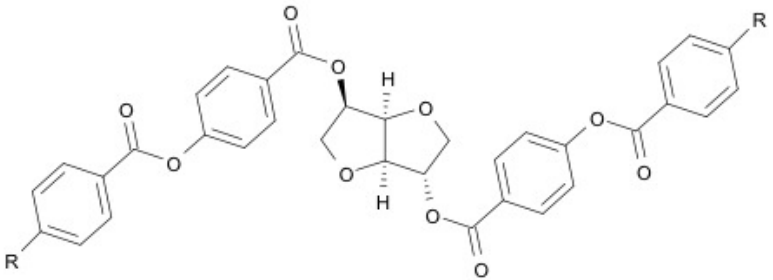

$\mathrm{R}=\mathrm{OMe}$ CAS RN $197663-64-2 \mathrm{HTP}=75 \mu \mathrm{m}^{-1}$ in E063

Figure 39: Method of Synthesis, using CD9 as an example 
to the clarity of the solution, only wavelength below $450 \mathrm{~nm}$ were scanned, and wavelength between 300nm to 450nm was used for baseline correction. Pure Hexanes without solute is used as reference, and scanning speed is $10 \mathrm{~nm} / \mathrm{sec}$.

\subsection{Results and Discussions}

The UV absorption spectrum of a particular molecule is a sensitive measure of the molecular conformation. All other factors being equal, a slight shift in molecular conformation can have a pronounced effect on the extinction coefficient. The measured absorbance of the sample is determined by the Beer Lambert equation:

$$
A=\varepsilon c l
$$

where $\mathrm{A}$ is the measured absorbance, $\varepsilon$ is the extinction coefficient $\mathrm{c}$ is the concentration and $l$ is the path length. We make these measurements on a single sample, so the total concentration and the path length remain constant. However, the relative concentration of the chair and boat conformations changr with temperature. Because their $\varepsilon$ is different at different wavelengths the changes in relative concentration are revealed in the changes in the measure A. The division of the spectra revealed this 
change in concentration with the magnitude of the difference determined by the difference in the $\varepsilon$ for the chair and boat conformations.

The concentration of CD6 in hexanes is $5.63 \times 10^{-5}(w t$.$) , and in CD9 a saturated$ solution in hexanes is used, due to its lower solubility. Figure 40 shows the UV absorption spectra for CD6 in Hexanes at room temperature and at 50C. The subtle differences in the spectra are the result of the similar but not identical absorption spectra of the chair and boat conformations. By dividing the spectra at each temperature by the room temperature spectra, we got the divided spectra, shown in Figure 41. These subtle changes are amplified in divided spectra. When temperature goes up, from $25^{\circ} \mathrm{C}$ to $55^{\circ} \mathrm{C}$, the absorption of the wavelength region around $270 \mathrm{~nm}$ increases; however, when the temperature drops back from $55^{\circ} \mathrm{C}$ back to room temperature, the absorption does not go back to its original level due to solvent loss. This trend could be easily read from the division plot, Figure 41 . When temperature goes up, all of the four plots from $25^{\circ} \mathrm{C}$ to $55^{\circ} \mathrm{C}$ almost intersect at the same spot. But after cooling down, the absorption spectra has a vertical shift toward higher absorption. As absorption is proportional to concentration, it means the concentration of the solute increases due to solvent evaporation. The same trend could be easily found in CD9, Figure 42 and Figure 43. 


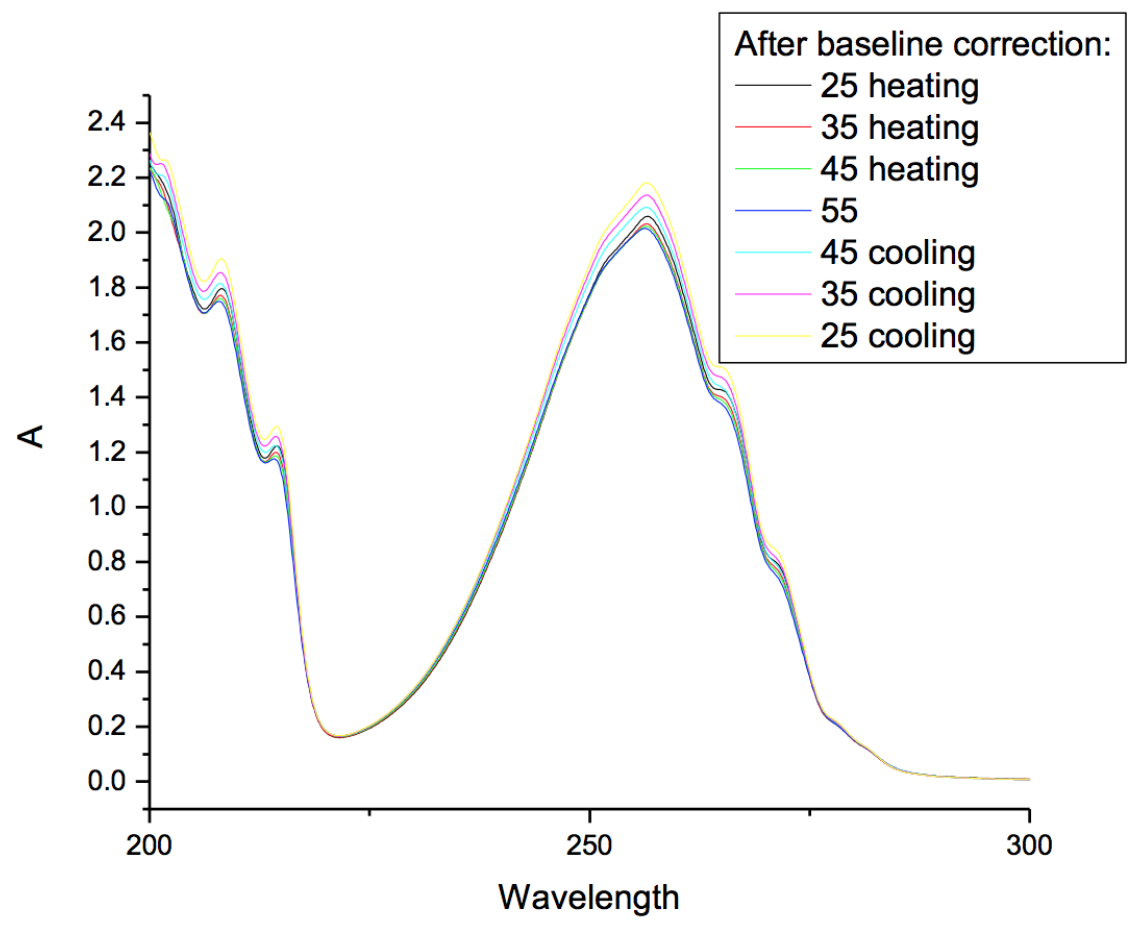

Figure 40: UV-Vis absorption spectroscopy of CD6 in hexanes temperature dependence 


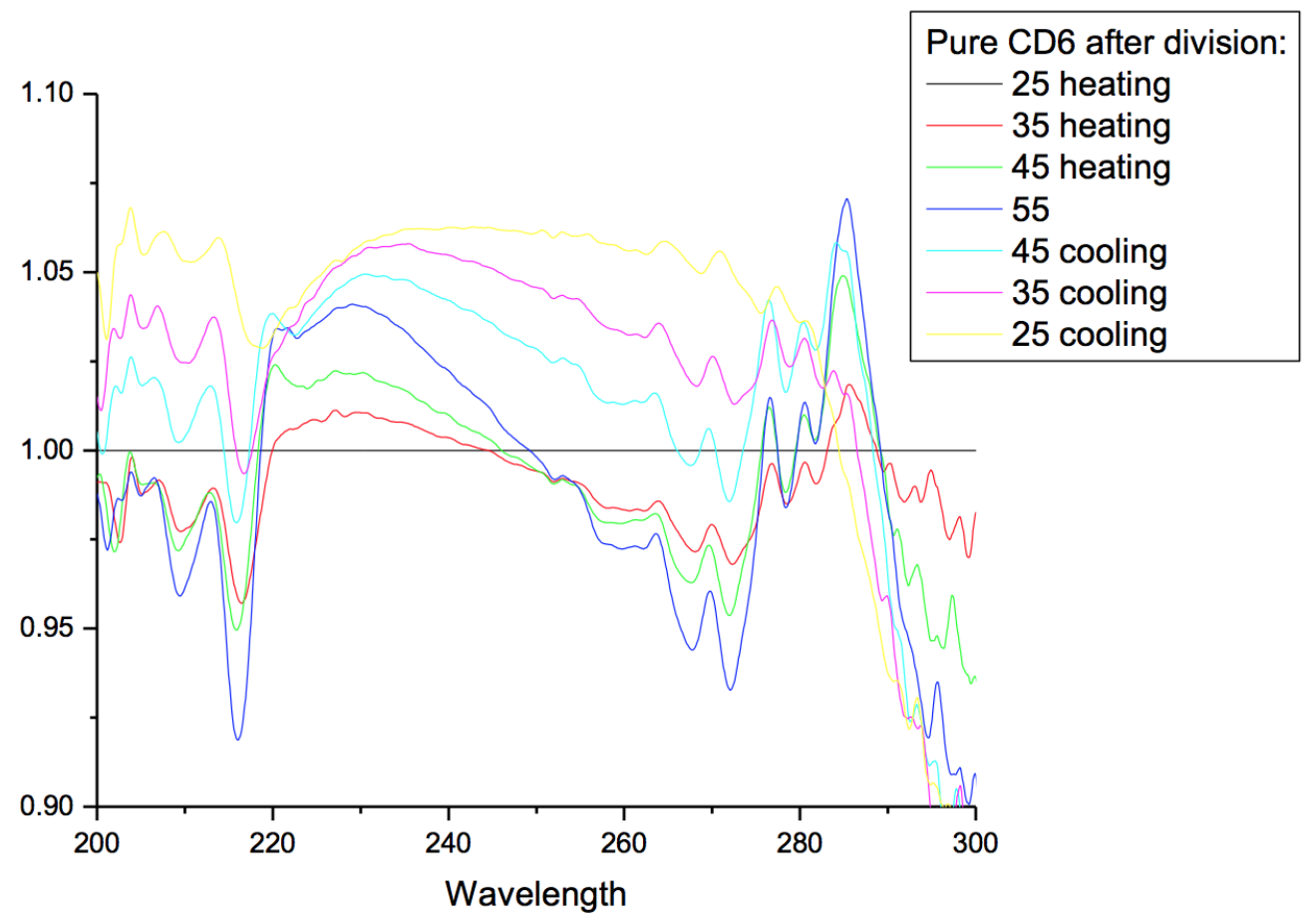

Figure 41: Differential absorption relative to absorbance at 0C for CD6 in Octane 


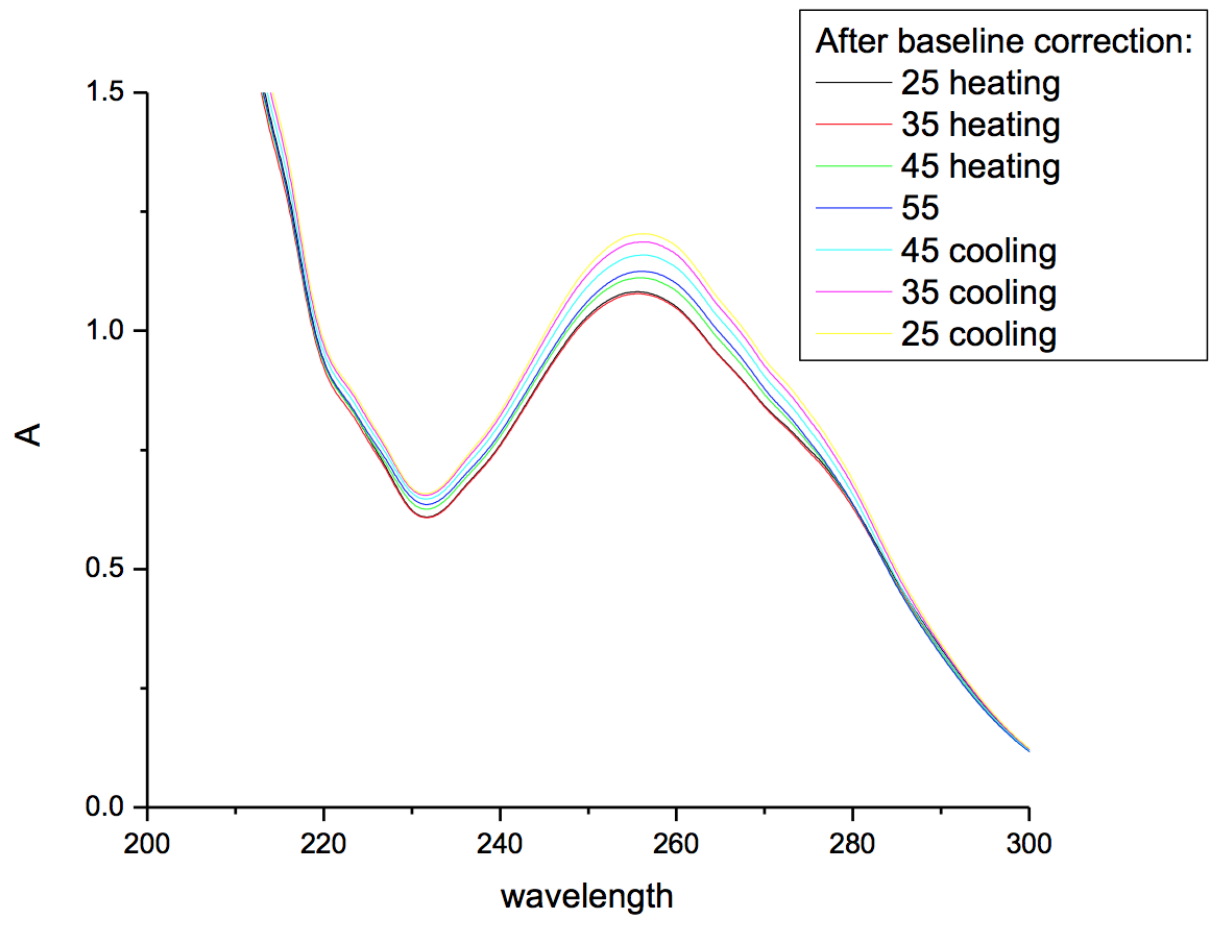

Figure 42: UV-Vis absorption spectroscopy of CD9 in hexanes temperature dependence 


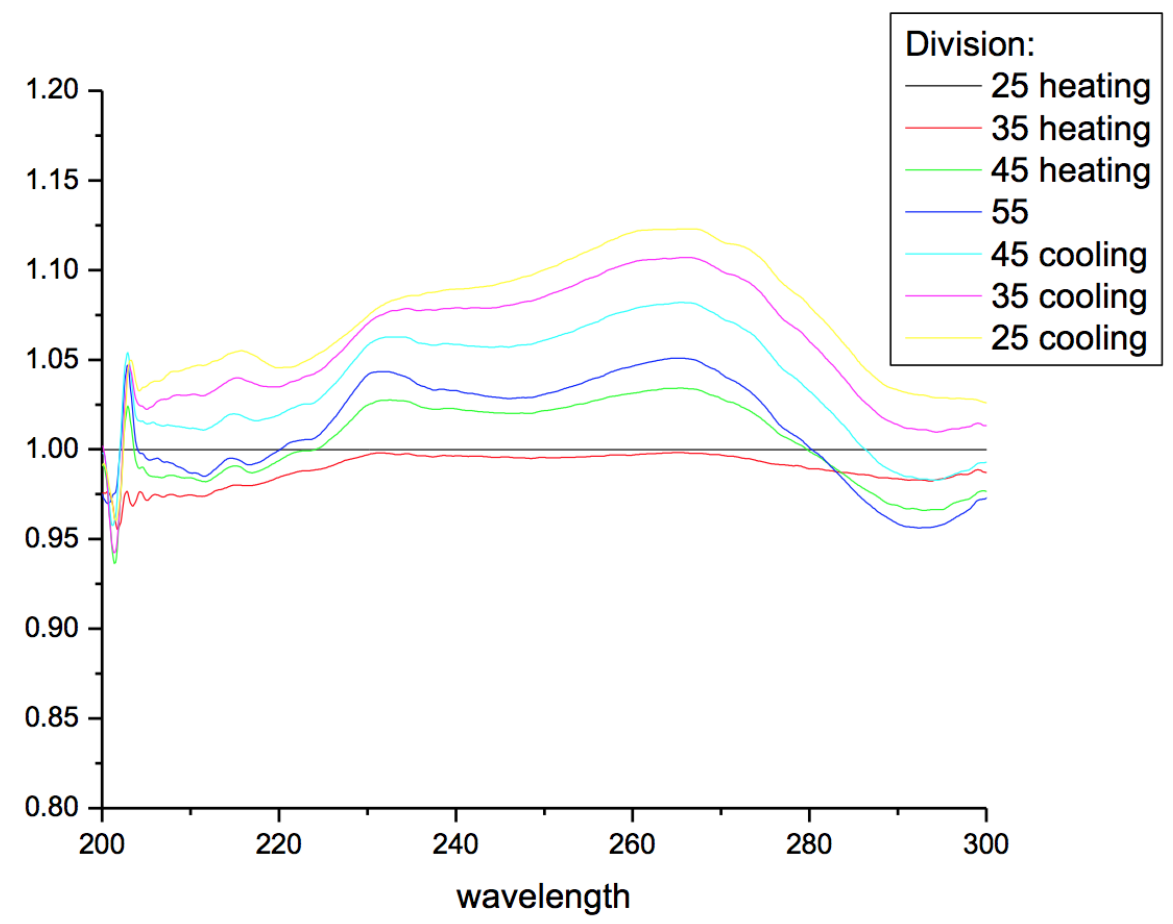

Figure 43: Relative UV-Vis absorption spectroscopy of CD9 in hexanes temperature dependence, using $25^{\circ} \mathrm{C}$ as baseline 
By weighting the cuvette before and after the test, we also confirmed around $10 \%$ weight change, which agrees with the UV-Vis absorption spectra. And due to the lower boiling point of the hexanes, in fact, some of the hexanes have boiling points as low as $58^{\circ} \mathrm{C}$. Other solvents, such as toluene, heptane, chloroform, and octanes, were tested. Finally, n-octane was chosen for its high boiling point, and high chiral dopant solubility.

Using chiral dopant dissolved in octane, we are able to obtain the absorption spectra change over a wider temperature range. Figure 44 shows the CD6 in octane UV-Vis spectra, vs temperature. The absorption spectra changes appear similar to hexanes solution. In order to see changes more clearly, $0^{\circ} \mathrm{C}$ spectrum is chosen as baseline. All the spectra is divided by $0^{\circ} \mathrm{C}$ spectrum, it clearly shows that all the spectra intersect at one point, which is at $238 \mathrm{~nm}$. If all the UV-Vis spectra goes through the same spot, this spot is called isosbestic point. Isosbestic point is the point that two conformers have the same extinction coefficient. The existence of isosbestic point indicates there are only two conformers exist in the system

The existence of a clear isosbestic point at about $238 \mathrm{~nm}$ clearly demonstrates the reliability of the measurement and the existence of only two predominant conformers of the CD6 molecule. The isosbestic point occurs because this is the point where the 


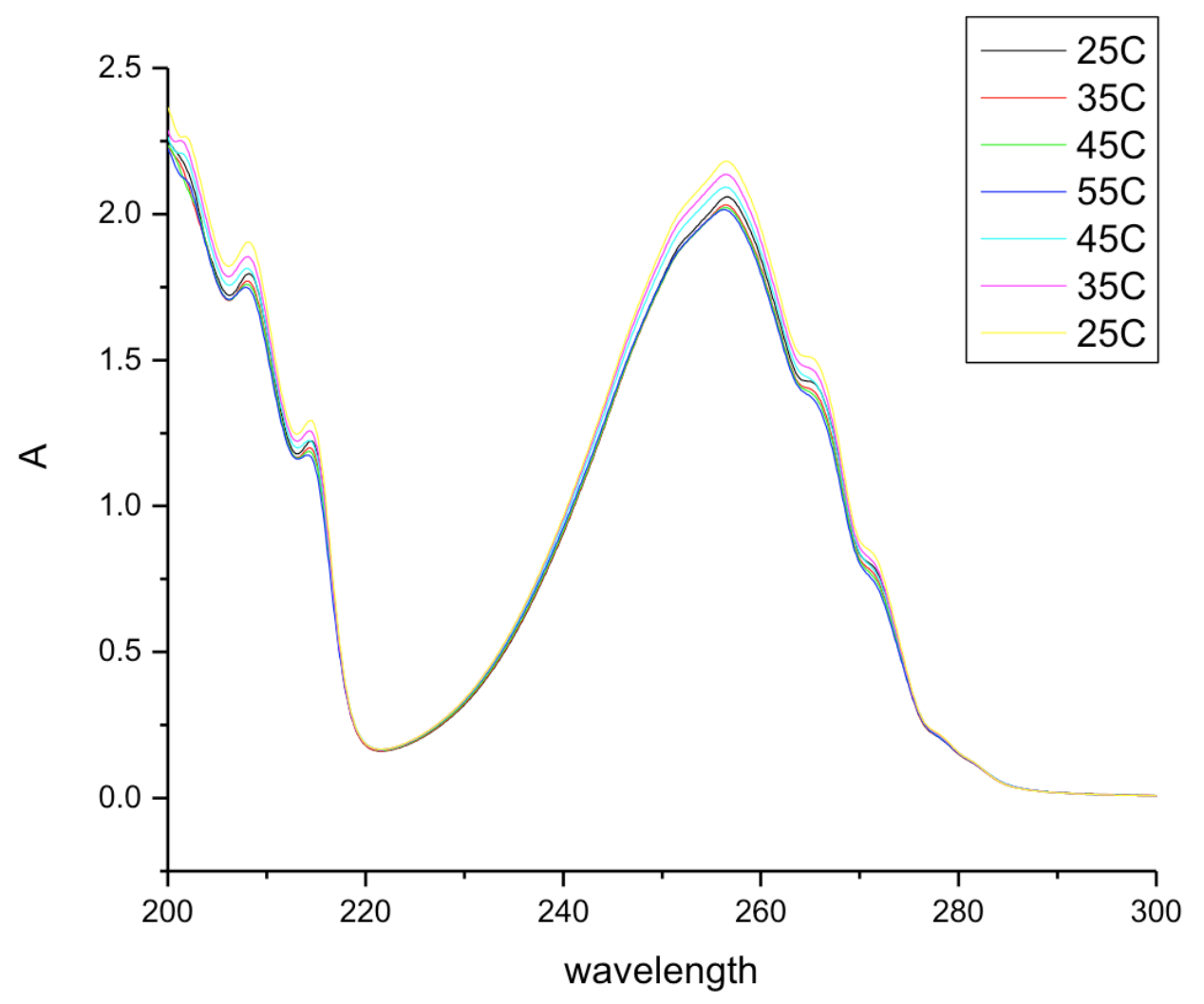

Figure 44: UV absorption spectrum of CD6 dissolved in octane as a function of temperature 


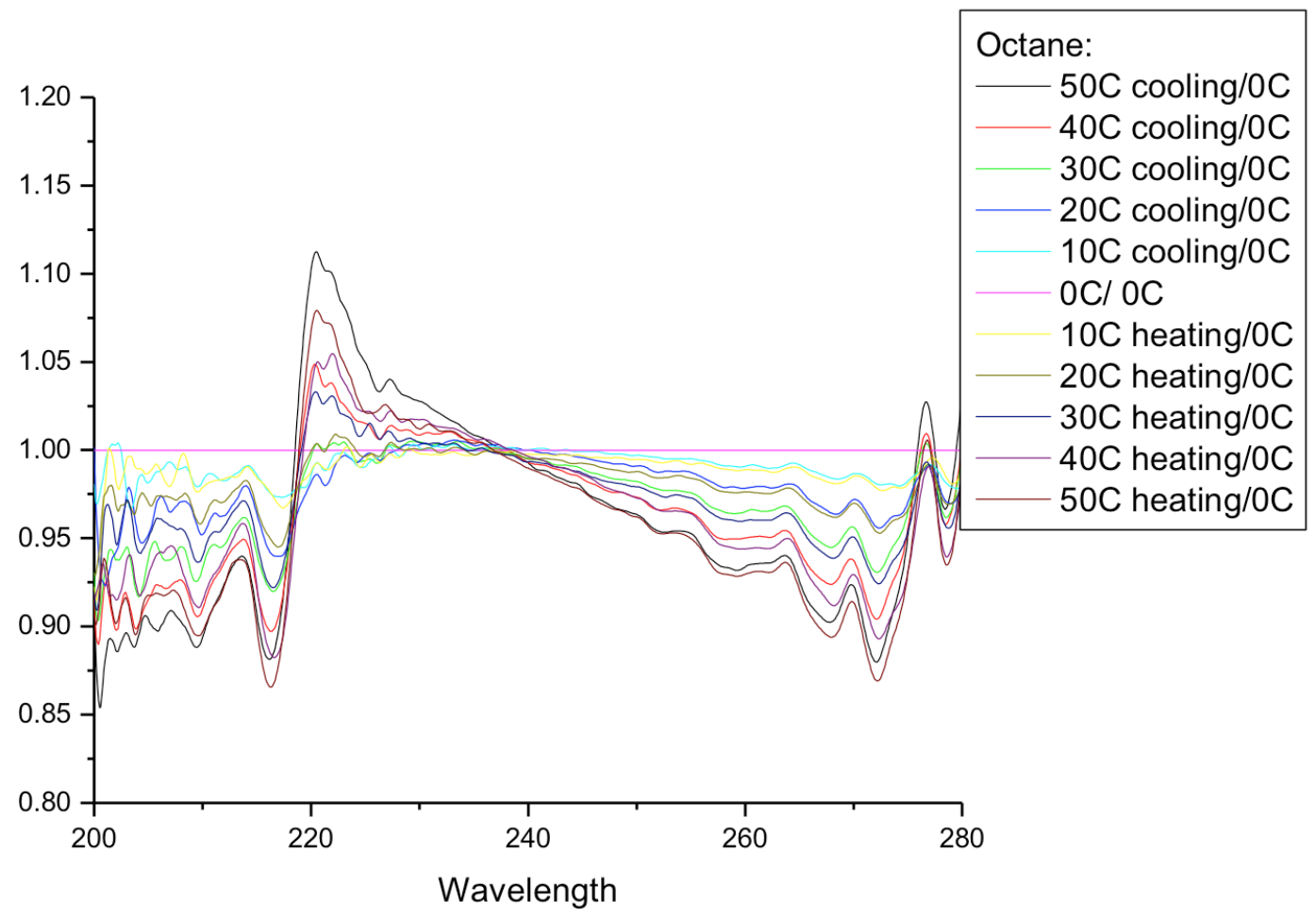

Figure 45: Differential absorption relative to absorbance at 0C for CD6 in Octane 

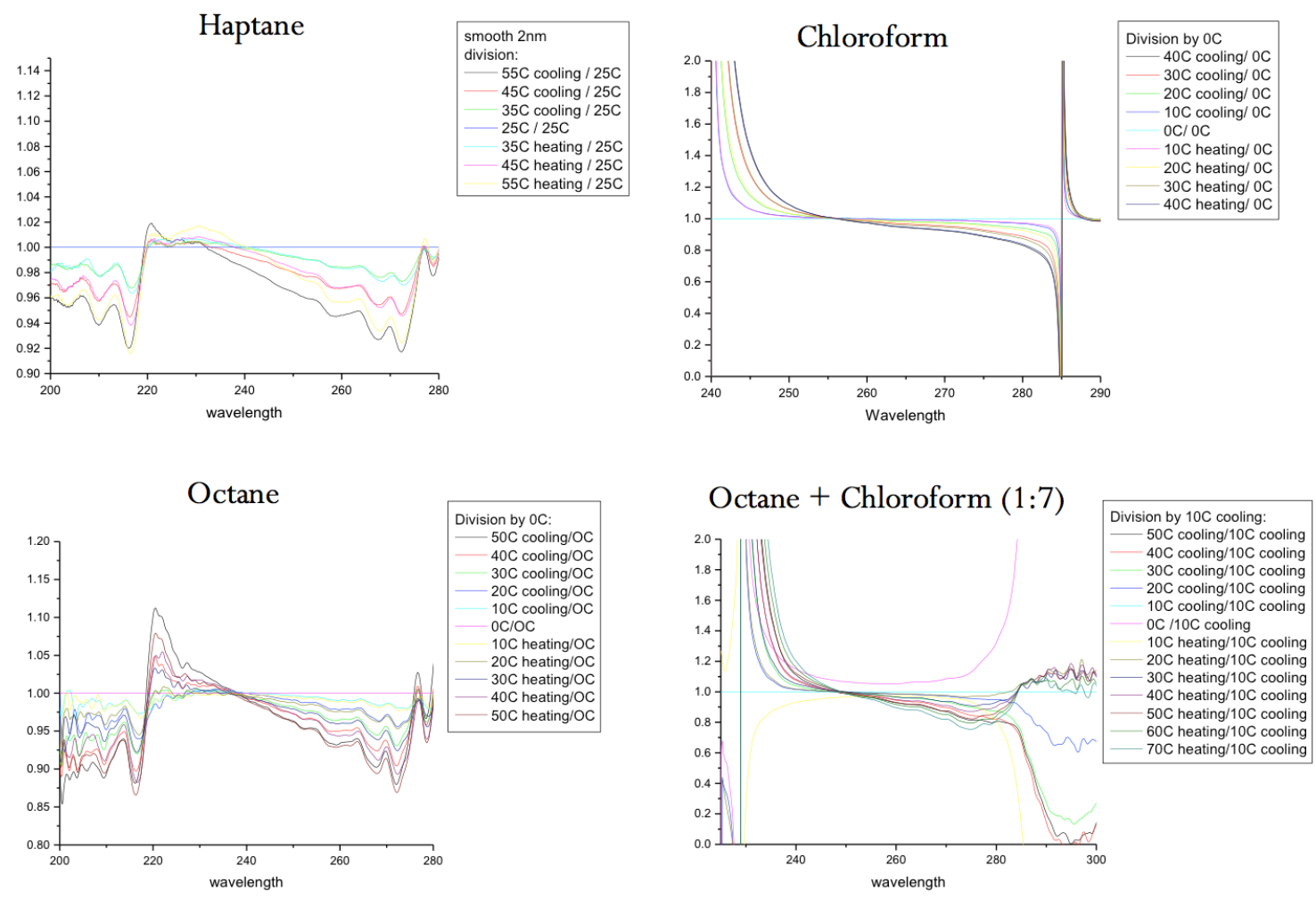

Figure 46: Isosbestic point exists in CD6 regardless of the solvent materials used. 
two conformers have the same extinction coefficient and the overall absorption spectrum is independent of the relative concentrations. Figure 46 shows the existence of isosbestic point is observed when CD6 dissolved in different solvents.

Similar behavior is observed for other members of this isosorbide series having alkyl chains with between 3 and 7 methylene carbons in the chain. Figure 47 shows the differential absorption spectra for this series and showing a clear isosbestic point for each member of the series. The isosbestic points are all around 238nm. However, when running the same test on CD9, yields a different behavior as shown in Figure 48. No clear intersect point, or isosbestic point found. The lack of the isosbestic point shows that there are not two predominant conformers. Molecular modeling of the CD9 (see in Figure 49) also produces distinctly different results from the shorter alkyl chain analogs with a more folded conformation seen for both the chair and boat like core structure and a smaller free energy difference between the two conformations.

As Figure 50 shows the deference of HTP temperature dependence for CD6 and CD9, CD9 appears to have very small temperature dependence, while CD6 shows large HTP variation while temperature changes.

Returning to the analysis of the CD6, it is apparent that a free energy difference 


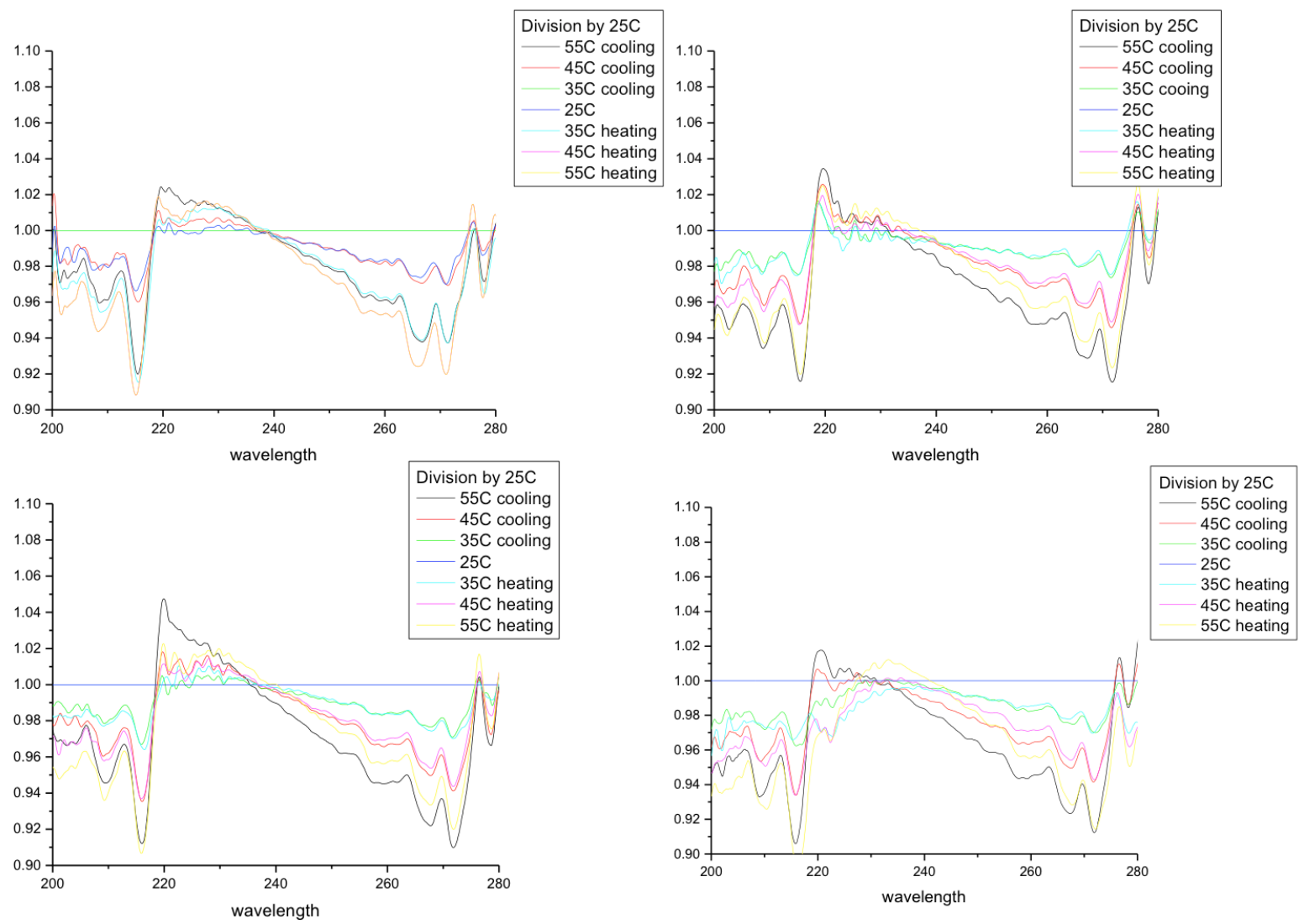

Figure 47: Differential absorption spectra of upper left: CD3, upper right: CD4, lower left, CD5 and lower right CD7 


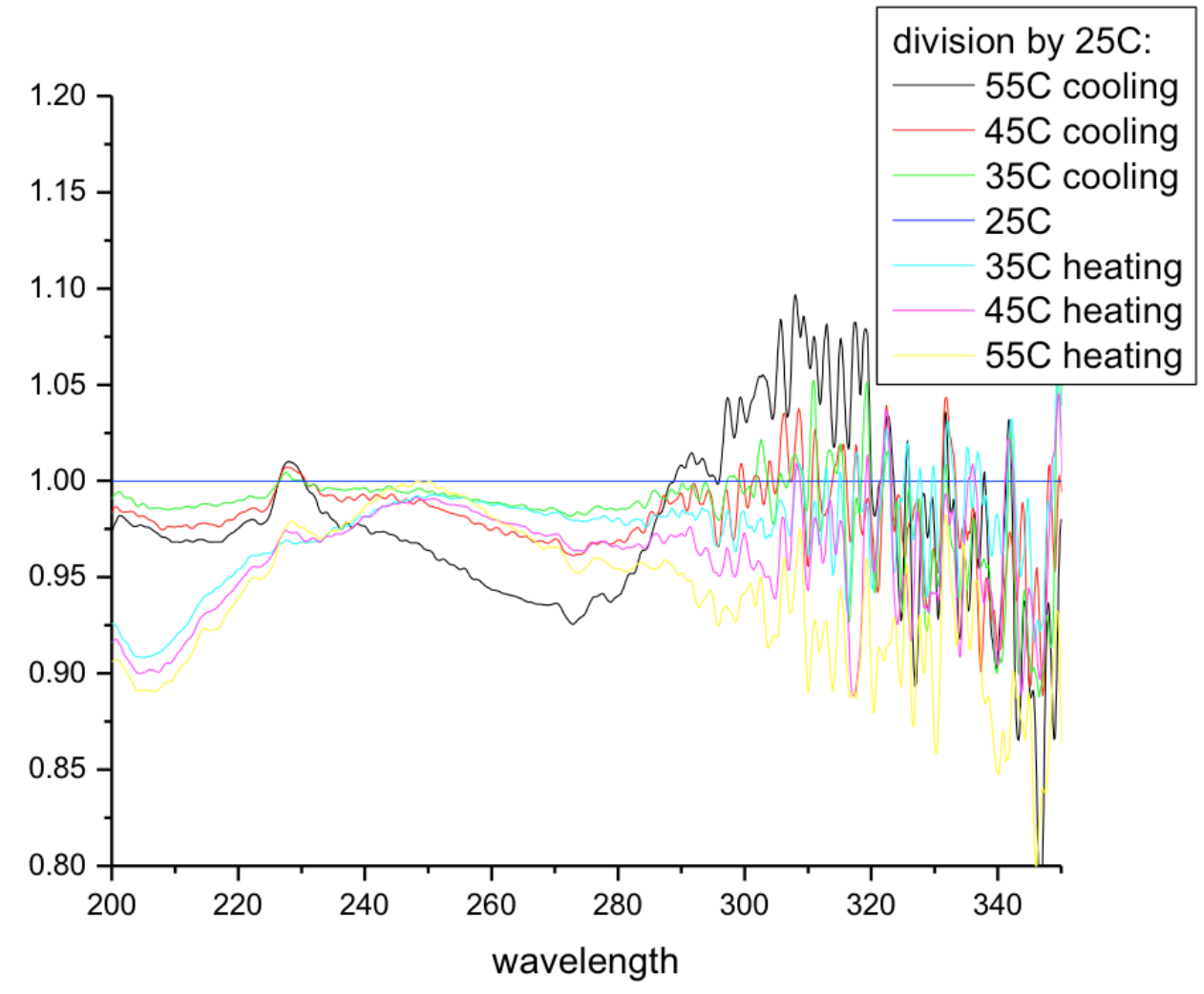

Figure 48: Differential absorption spectra as a function of temperature for CD 9 

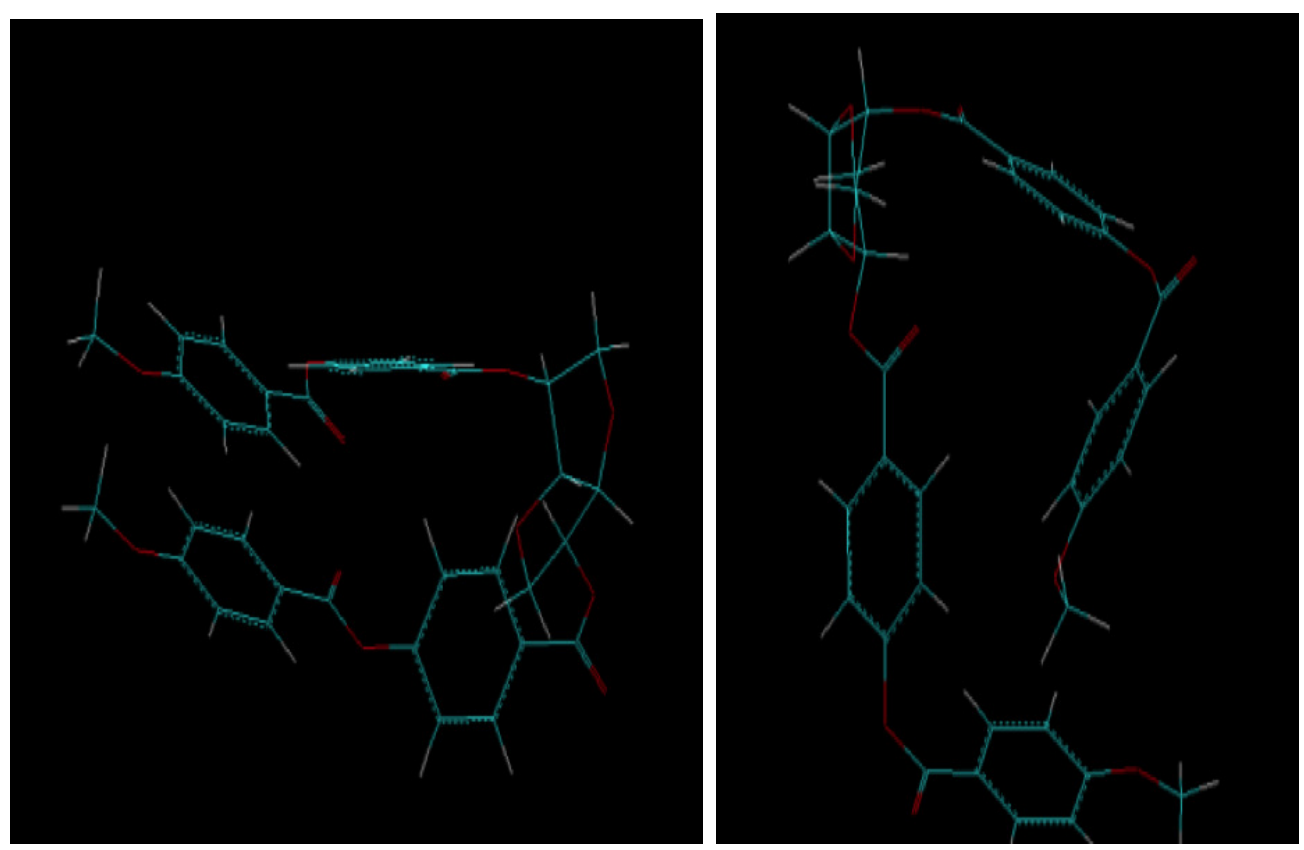

Figure 49: CD9: molecular model for the structure of the boat (left) and chair core conformation (right) 


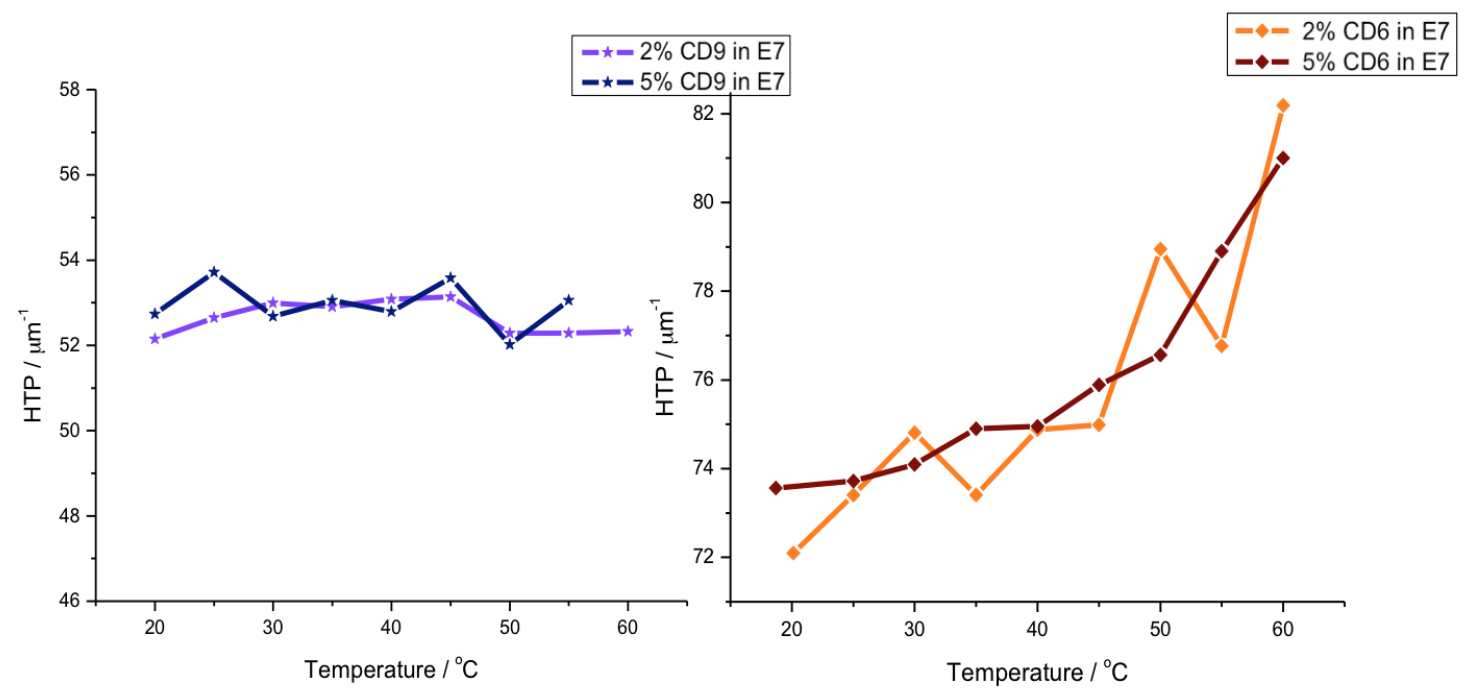

Figure 50: HTP temperature dependence of CD6 and CD9 


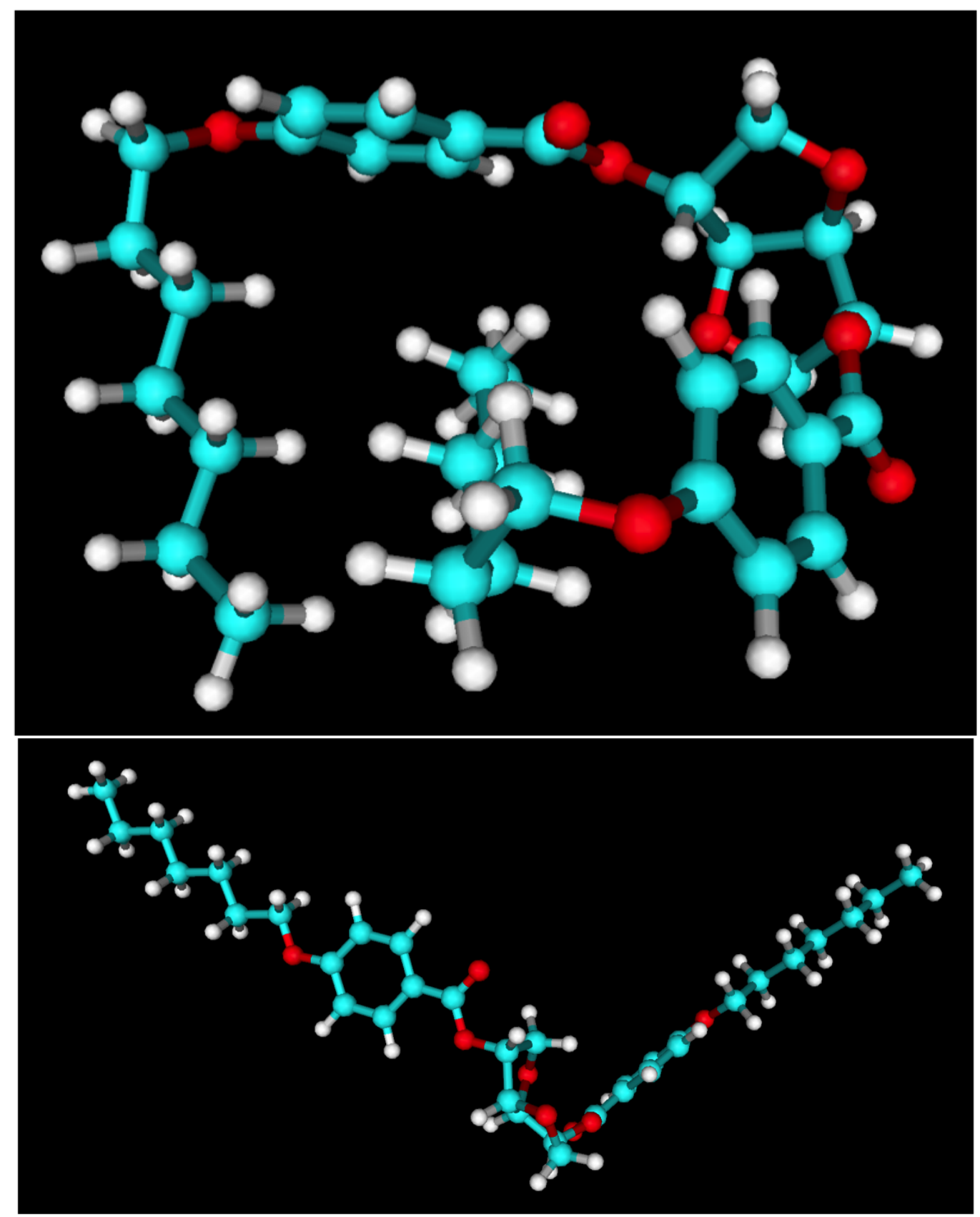

Figure 51: Predominant molecular conformations of CD6 
between of the boat and chair conformation of $3.5 \mathrm{kcal} / \mathrm{mol}$ will result in a large change in the population distribution as the temperature is raised from room temperature to $50^{\circ} \mathrm{C}$. The 5 member rings at the core of the CD6 exist in two conformations termed the twist and envelope structure. The two rings of the isosorbide core exist in two predominant conformations termed the boat and chair, analogous to the structure of cylcohexane. Molecular modeling of the CD6 shows that the boat and chair conformations are very different, the boat conformation taking on a more folded spherical overall shape and the chair conformation having a more propeller like shape. These large changes in overall structure would be expected to produce distinctly different HTPs. Figure 51 shows the molecular models of these two conformations of CD6. The molecular model predicts that the boat and chair conformer of CD 6 vary in free energy by about $3.5 \mathrm{kcal} /$ mole for the molecule considered in vacuum.

The exact conformation of the chiral additive will depend on the liquid crystal host and will differ somewhat from the model. However, the model provides a good starting point for understanding the temperature dependence of the HTP of CD6. As noted above, the distribution of the two conformers will depend on the difference in their free energy based on a simple Boltzmann distribution. The fractional population distribution of different conformers can be written as 


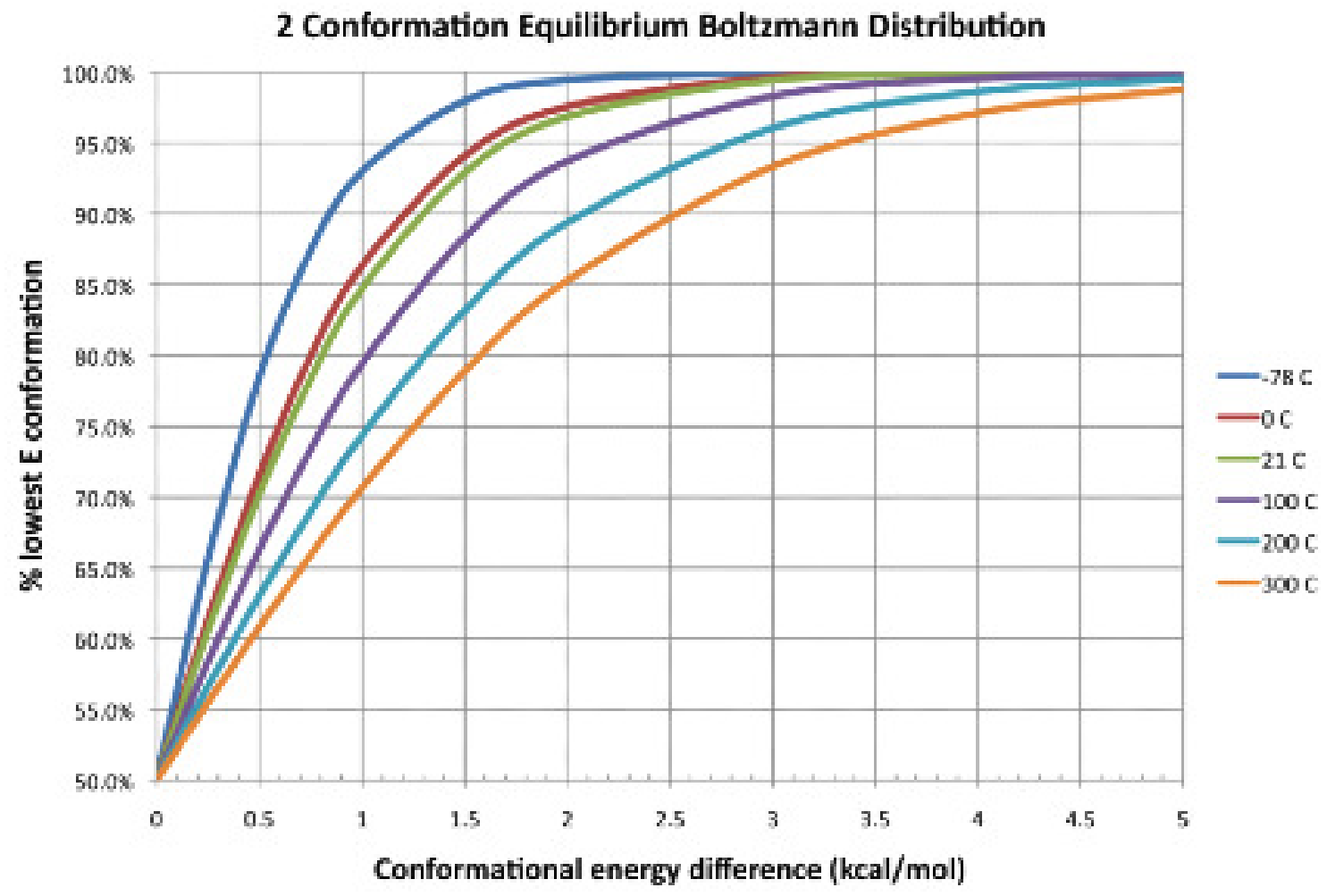

Figure 52: Relative distribution between 2 conformers based on temperature and the difference in their free energy 


$$
\frac{N_{i}}{N_{\text {total }}}=\frac{e^{-E_{r e l} / R T}}{\sum_{k=1}^{N_{\text {total }}} e^{-E_{k} / R T}}
$$

Figure 52 shows the population distribution between two conformers based on the difference in their free energy. If there is a large difference $(>5 \mathrm{kcal} / \mathrm{mol})$ in the free energy, essentially only one conformer exists at practical temperatures. Conversely, if there is only a small difference in the free energy $(<0.5 \mathrm{kcal} / \mathrm{mol})$ the two conformers have a nearly equal distribution that changes little over practical temperature ranges. If there is a way of slowing down the switches between the two conformers, one can capture the behavior of each single conformer. Low temperature can slow down the conformational switch, and NMR can capture a conformer's information at a very short time.

\subsubsection{NMR Spectroscopy}


If the temperature can reach low enough, and the switches between the two conformations are slow enough, NMR is a method that can help capture the two conformational states. Then relative concentration can be calculated, as well as HTP for each conformers. In order to and simplify the problem, and lower the number of protons in similar chemical environment, CD3 is chosen to mix with deutero-octane/deuteron chloroform blend solvent.

Proton NMR spectra were obtained using deutero chloroform blend as the solvent. Proton NMR spectra were measured between room temperature and $-80^{\circ} \mathrm{C}$. In the chloroform solution is used in for this test, unlike the other chiral dopants in our list, CD3 has only $6 \mathrm{CH}_{3} \mathrm{O}$ protons, no proton on carbon chain. Protons are marked in Figure 53. This feature helps us simplify the problem and is easier to interpreted spectra. NMR frequency is $500 \mathrm{MHz}$. TMS is used as the reference.

Figure 54 shows NMR spectra vs temperature change of protons on the isosorbide core rings. Figure 55 shows NMR spectrum at $25^{\circ} \mathrm{C} .8$ protons on both phenyl groups appear at lower field side of the spectrum due to the deshielding effect of phenyl groups. And because of oxygen's high electronnegativity, proton 2 and 5 appear successively in the $5.4 \mathrm{ppm}$ region, and proton 4 and 3 are next to them towards the higher field. The rest of the 4 protons $1 \mathrm{a}, 1 \mathrm{~b}$, and $6 \mathrm{a}$, and $6 \mathrm{~b}$ all overlap in the 


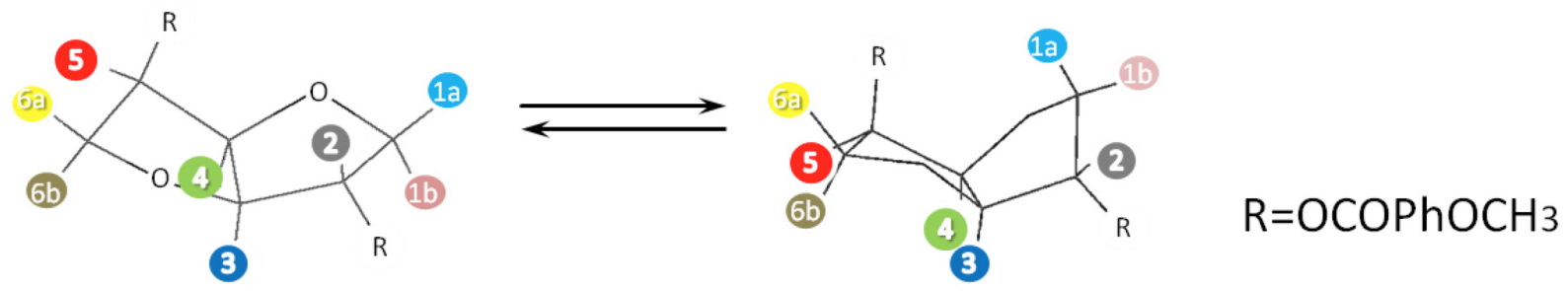

Figure 53: Chair and boat structure of CD3 with proton marks 


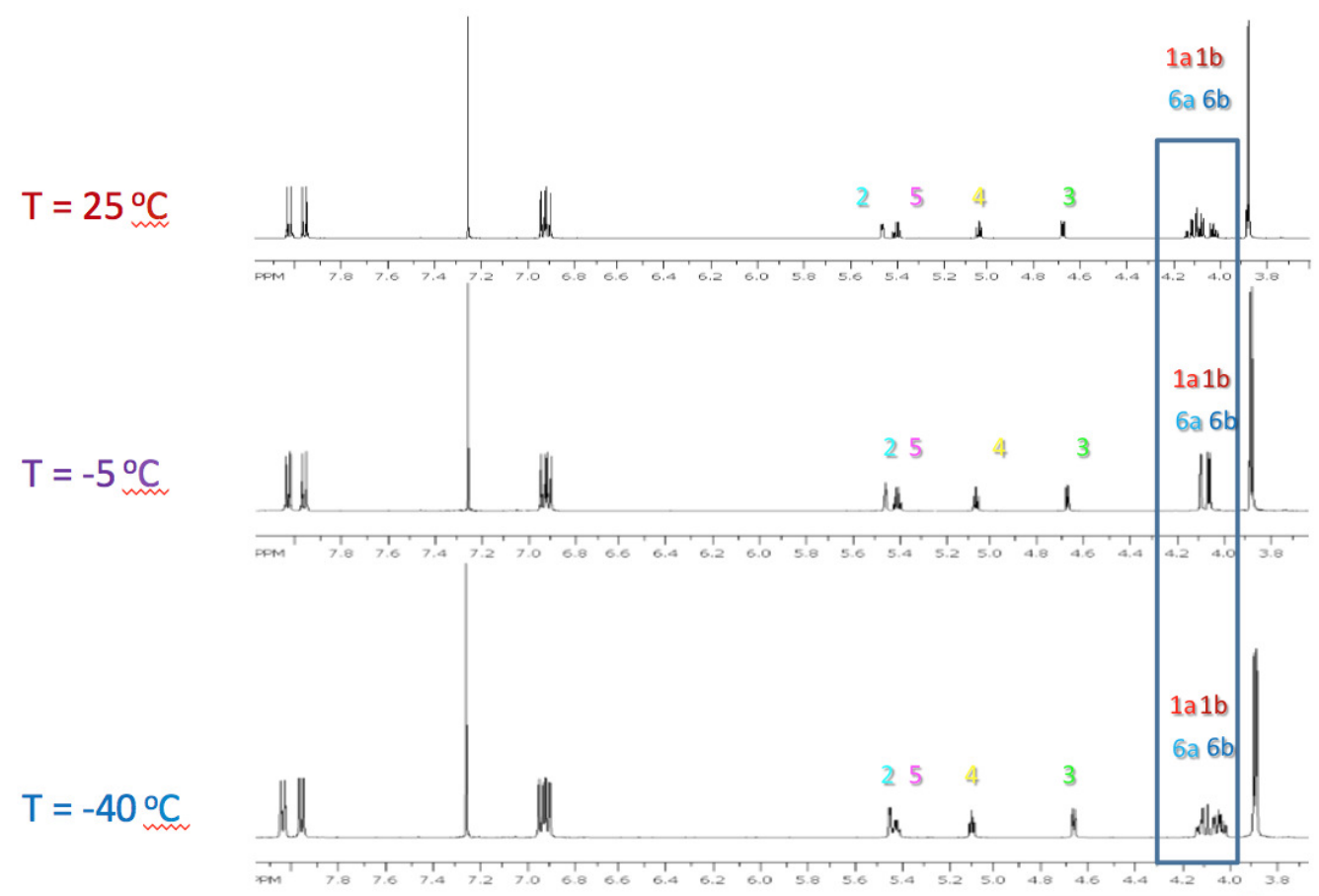

Figure 54: NMR spectra vs temperature change of protons on the isosorbide core rings 
4.0 to $4.2 \mathrm{ppm}$ region, due to their similar chemical environment, and large coupling effect. Finally, the methyl groups appear at highest field.

Figure 56 and Figure 57 show separated peaks for 1a, 1b, 6a, 6b. Figure 58 shows more clearly that when temperature goes down, the peaks spread out more. Ideally, once the temperature gets low enough, all the CD3 molecules will be "locked" at conformer with lower energy state, instead of switching back and forth. Then we should be able to calculate the relative concentration of the two conformers and get the HTP for each of them. Figure 58 and Figure 59 show temperature dependence of the NMR spectra of proton 1a, 1b, 6a, 6b, and protons $2,5,4,3$, respectively.

Figure 60, Figure 61 and Figure 62 show NMR signal change with temperature of protons 1a, 1b, 6a, 6b, 2,5, and 3, 4, respectively. During the temperature change, the NMR signal for each proton on the ring is moving between two states, which indicates the distribution of the two states changes due to temperature.

In this case the ratio of the geminal/vicinal coupling constants of the two methylene carbons in the bridged 5 member rings of the isosorbide core changes as a function of temperature confirming the change in conformer ratio, although in experiment $-50^{\circ} \mathrm{C}$ or $-80^{\circ} \mathrm{C}$ is not low enough to froze out the conformers. See Figure 63 [78]. 


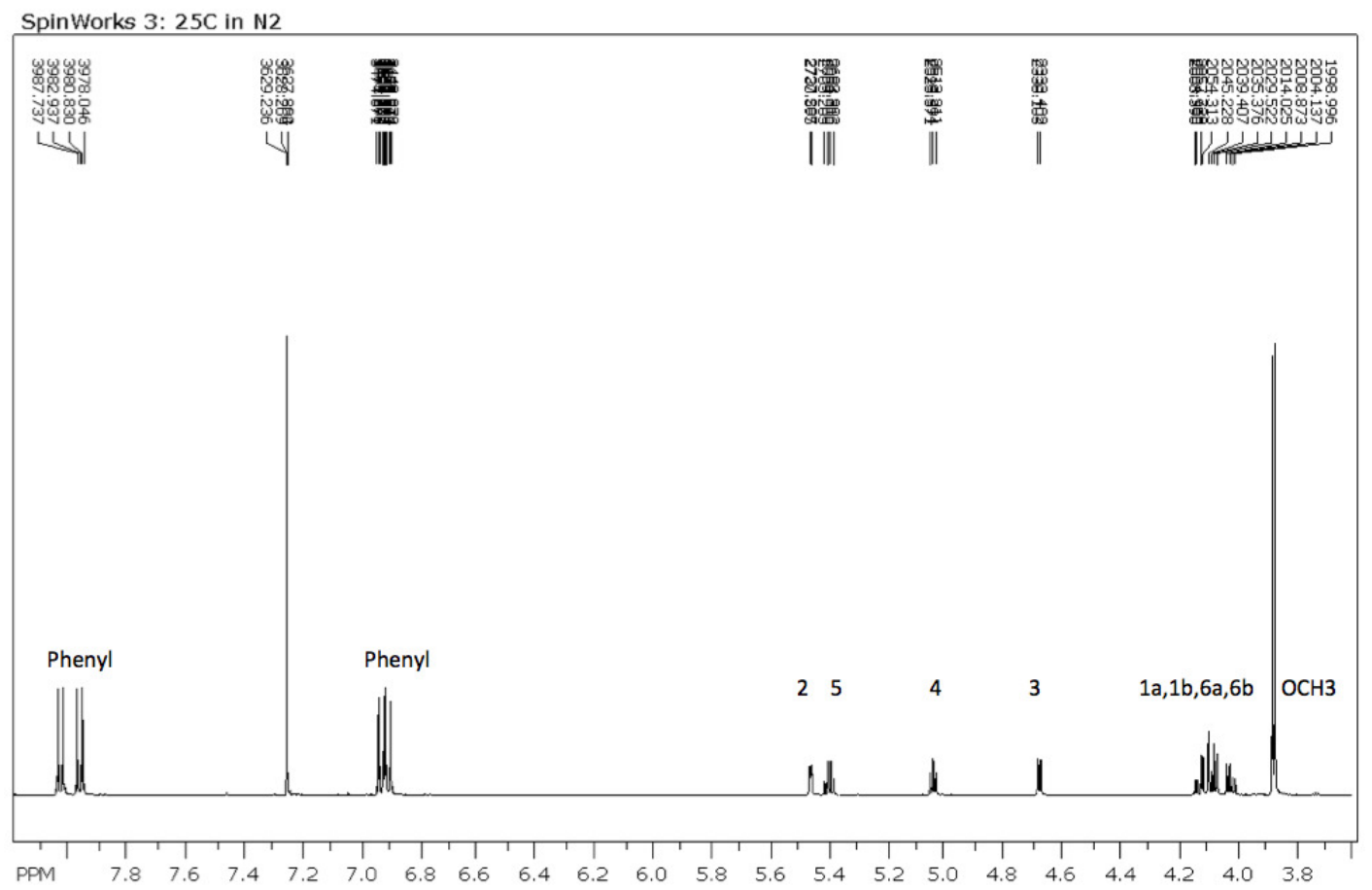

Figure 55: NMR spectra of CD3 at room temperature 


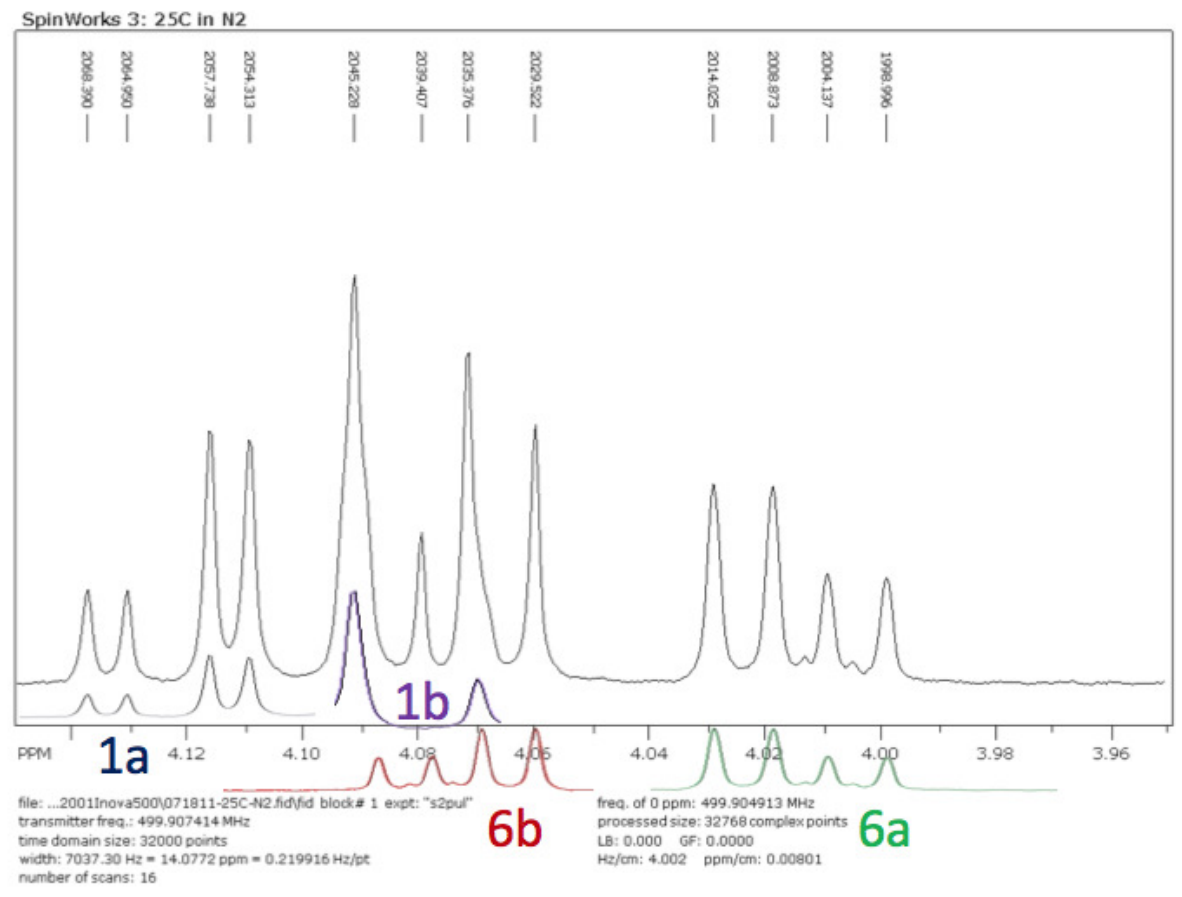

Figure 56: NMR spectra of of proton 1a, 1b, 6a, 6b at room temperature 


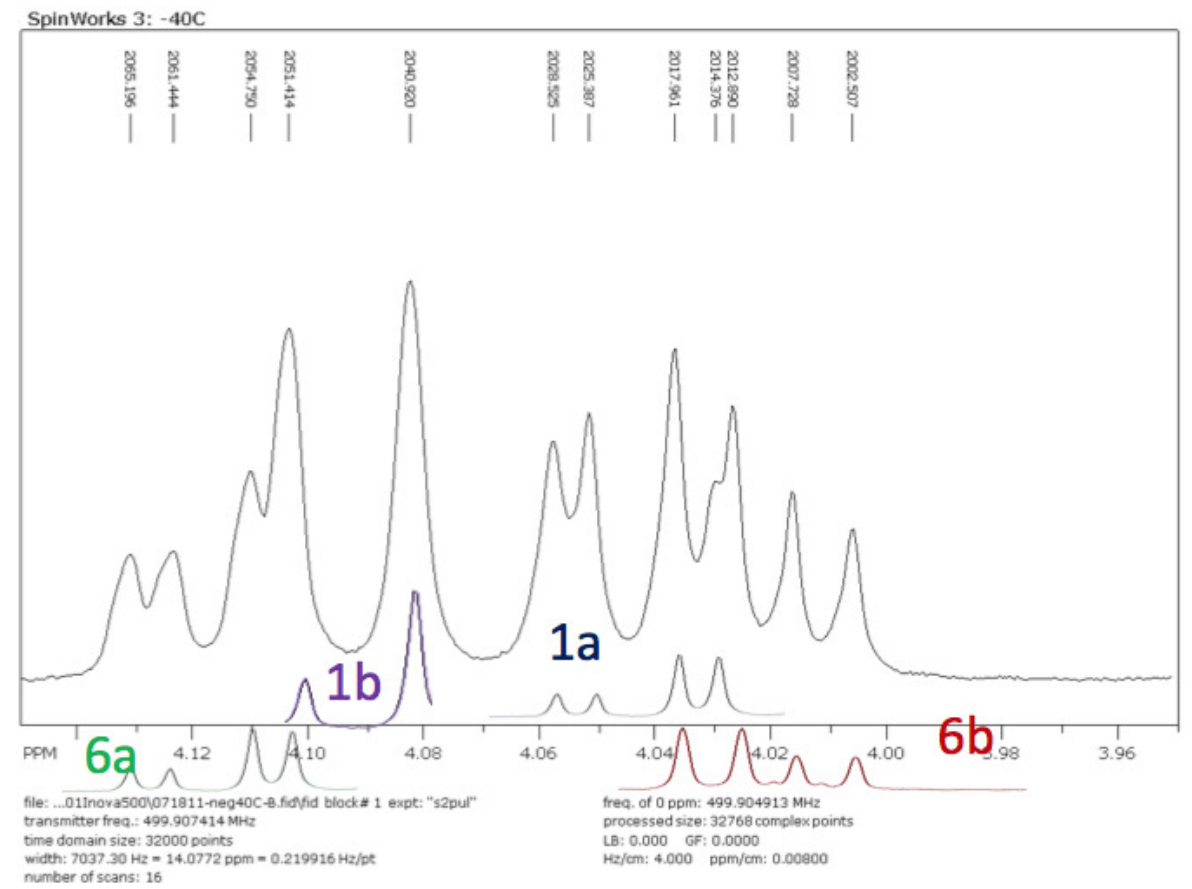

Figure 57: NMR spectra of of proton $1 \mathrm{a}, 1 \mathrm{~b}, 6 \mathrm{a}, 6 \mathrm{~b}$ at $-40^{\circ} \mathrm{C}$ 


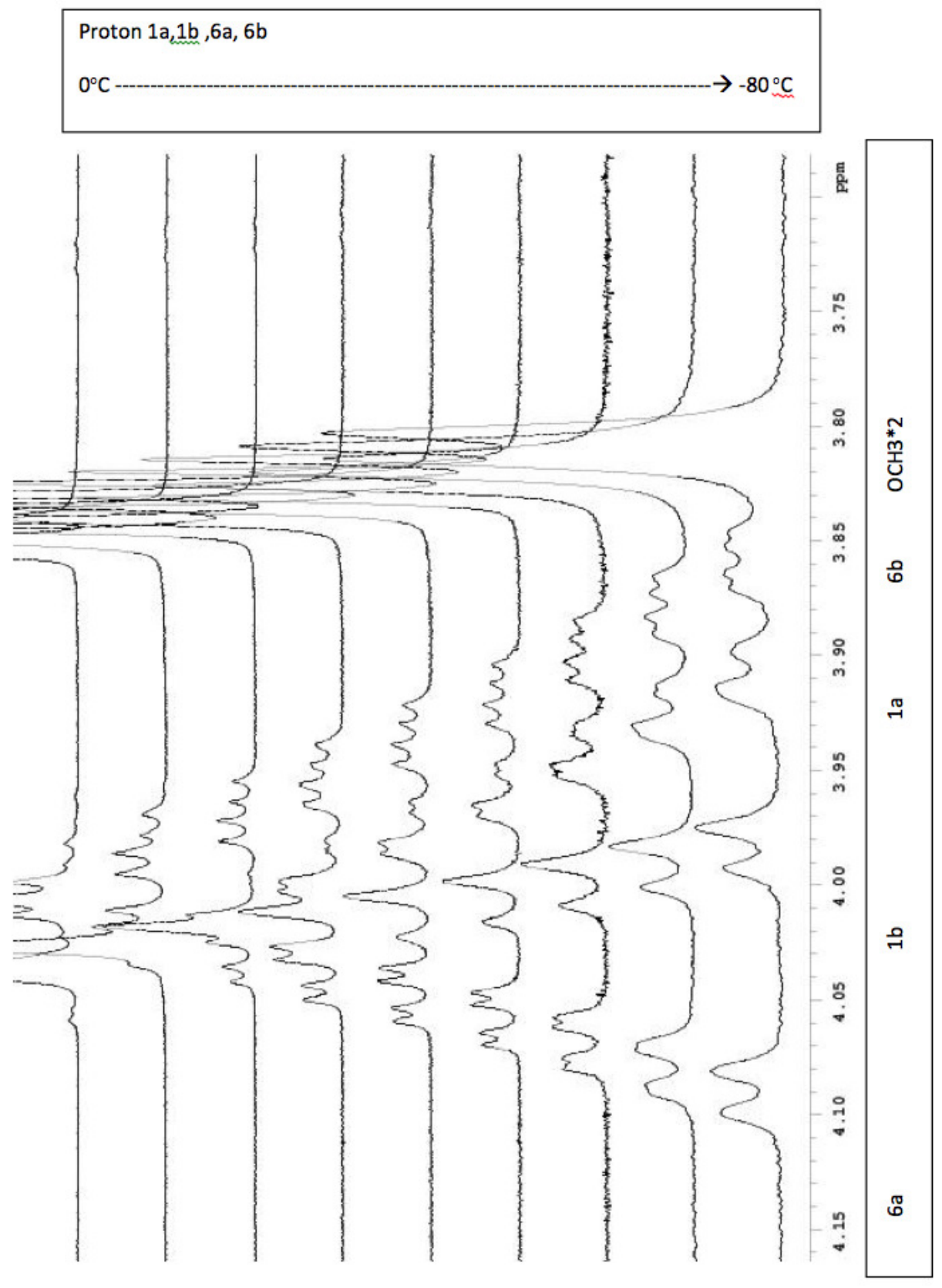

Figure 58: NMR spectra of of proton 1a, 1b, 6a, $6 \mathrm{~b}$ by temperature change 


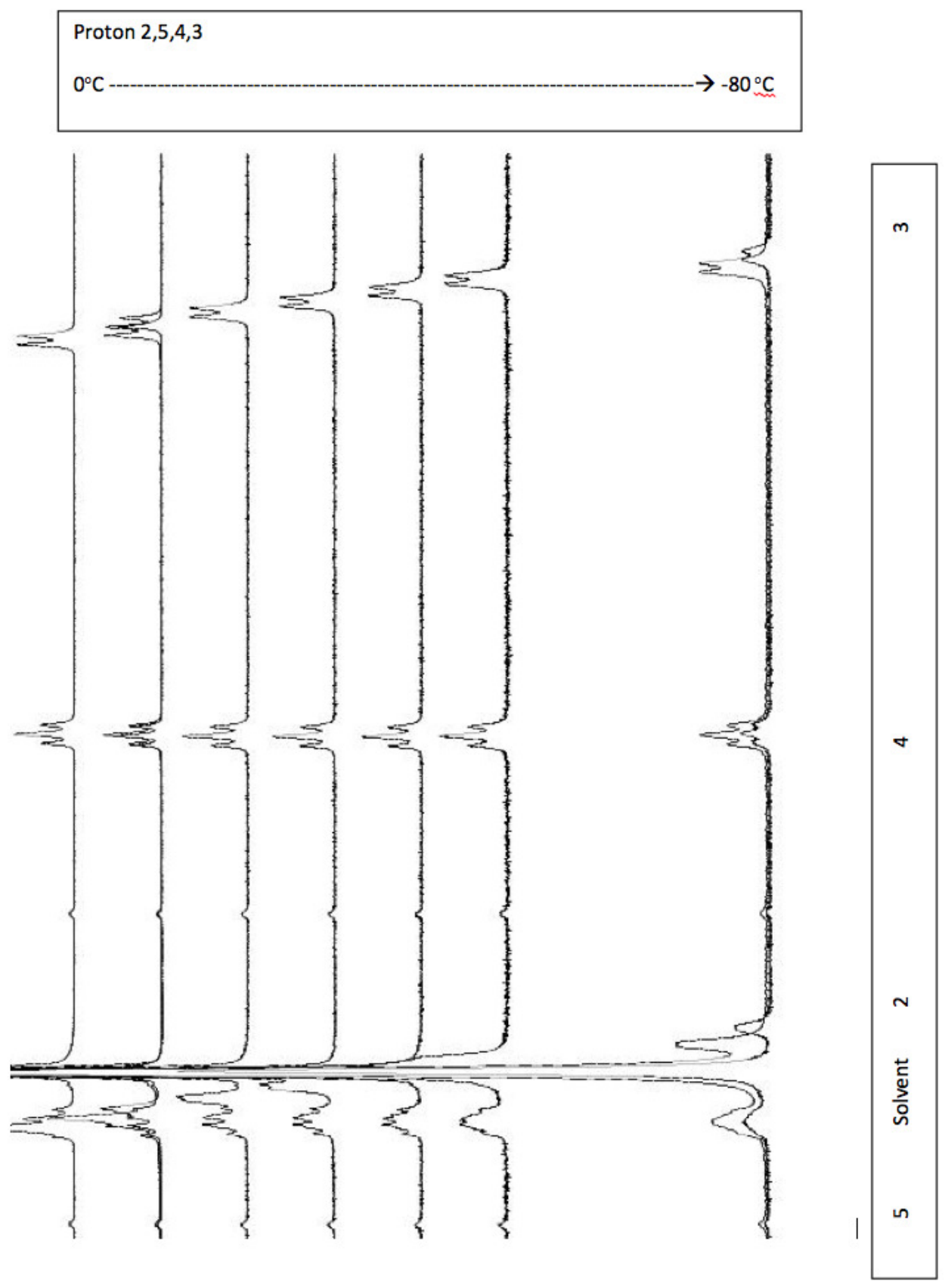

Figure 59: NMR spectra of of proton 2, 5, 4, 3 by temperature change 

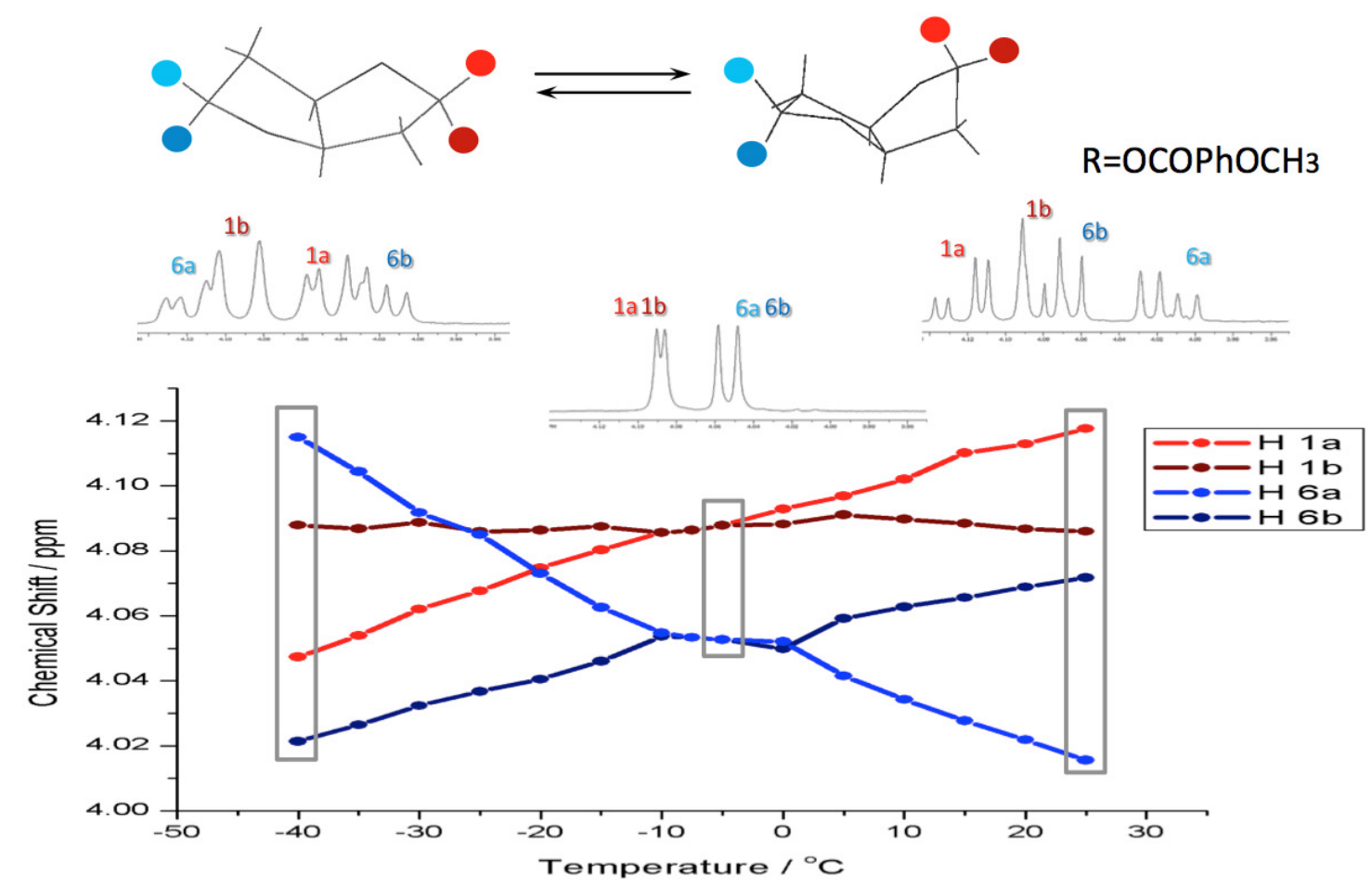

Figure 60: NMR signal verses temperature of protons 1a, 1b, 6a, 6b 

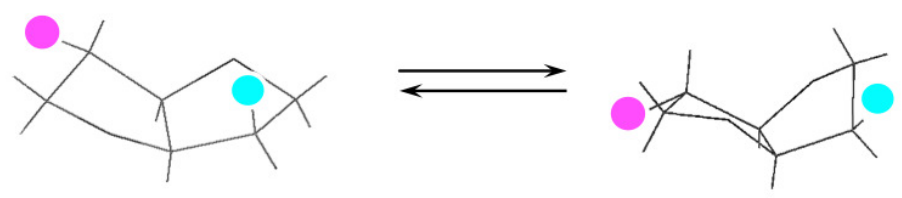

\section{$\mathrm{R}=\mathrm{OCOPhOCH}$}

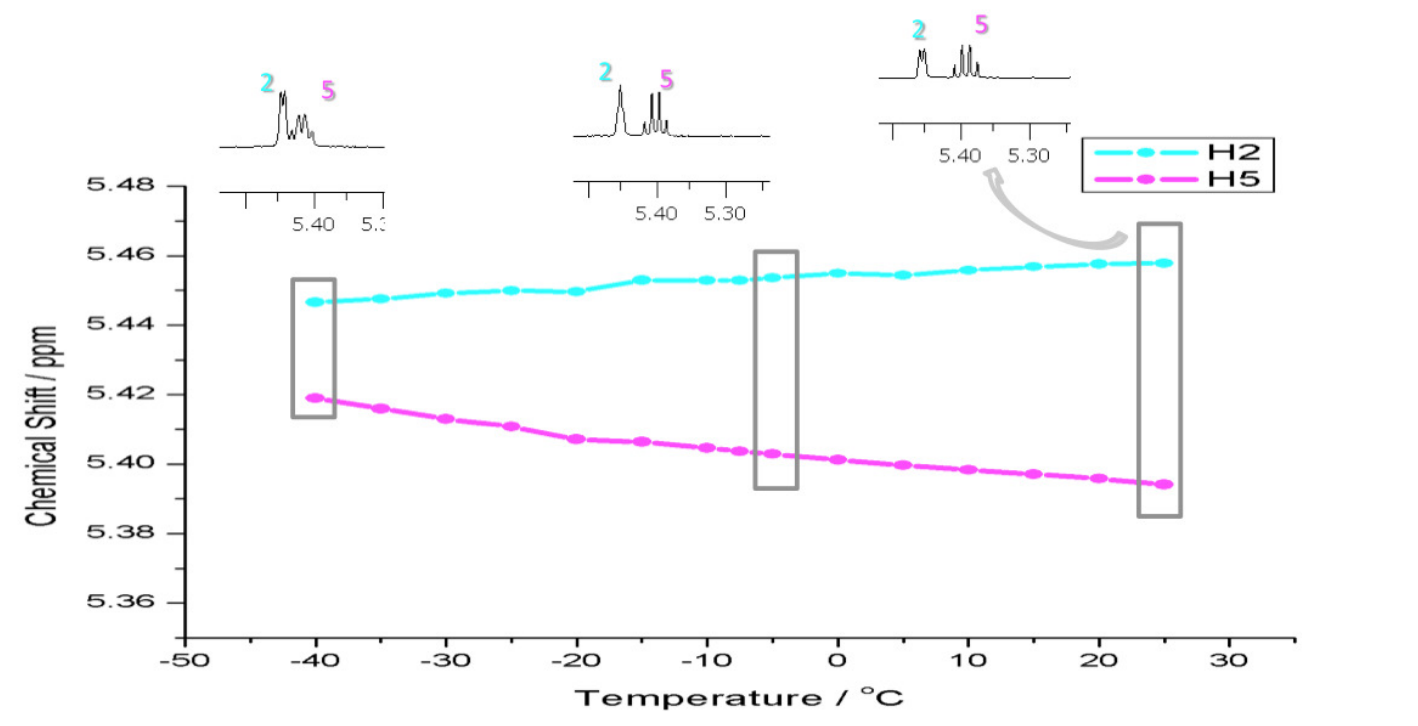

Figure 61: NMR signal verses temperature of protons 2 and 5 


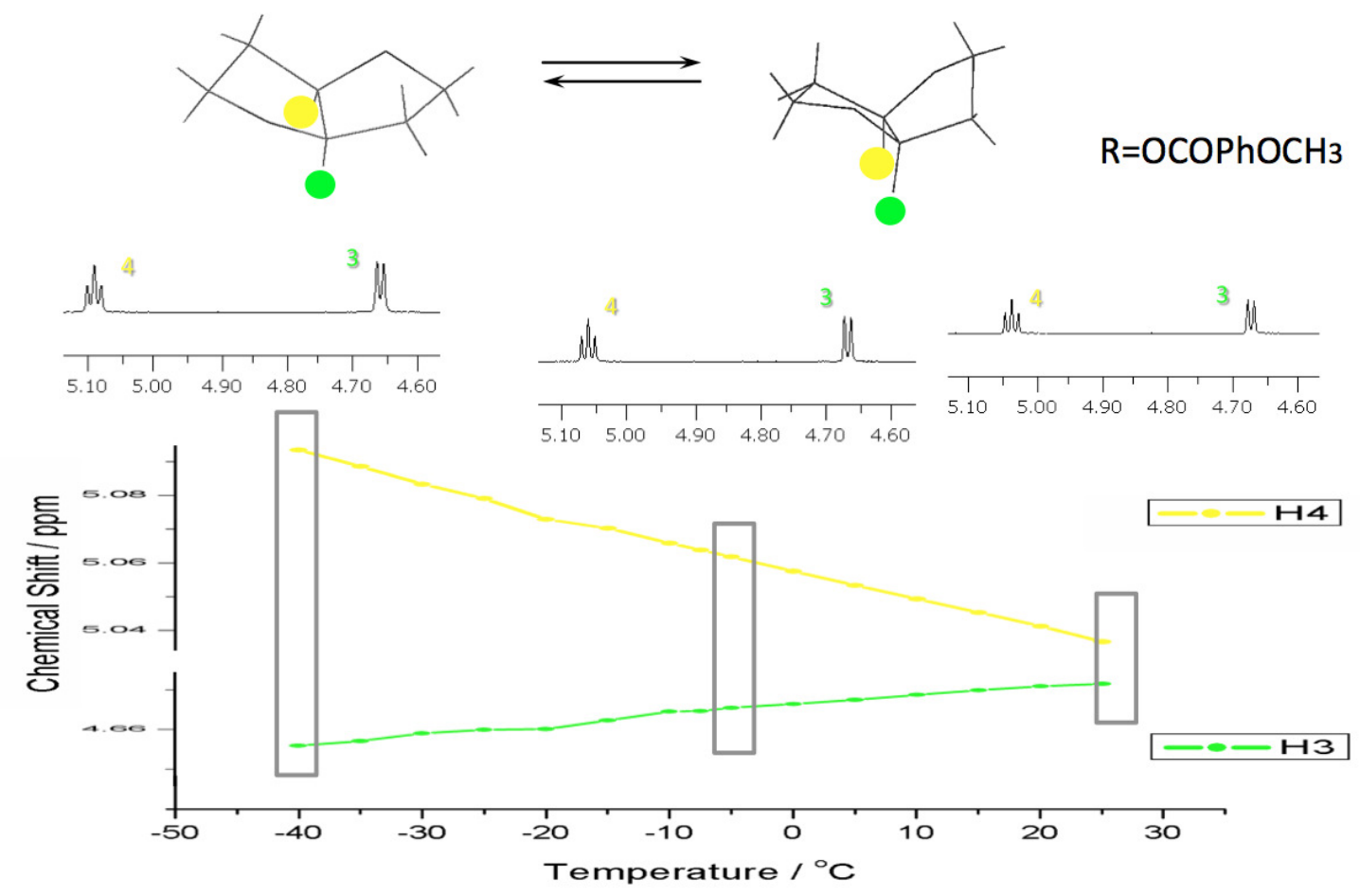

Figure 62: NMR signal change by temperature of proton 3 and 4 

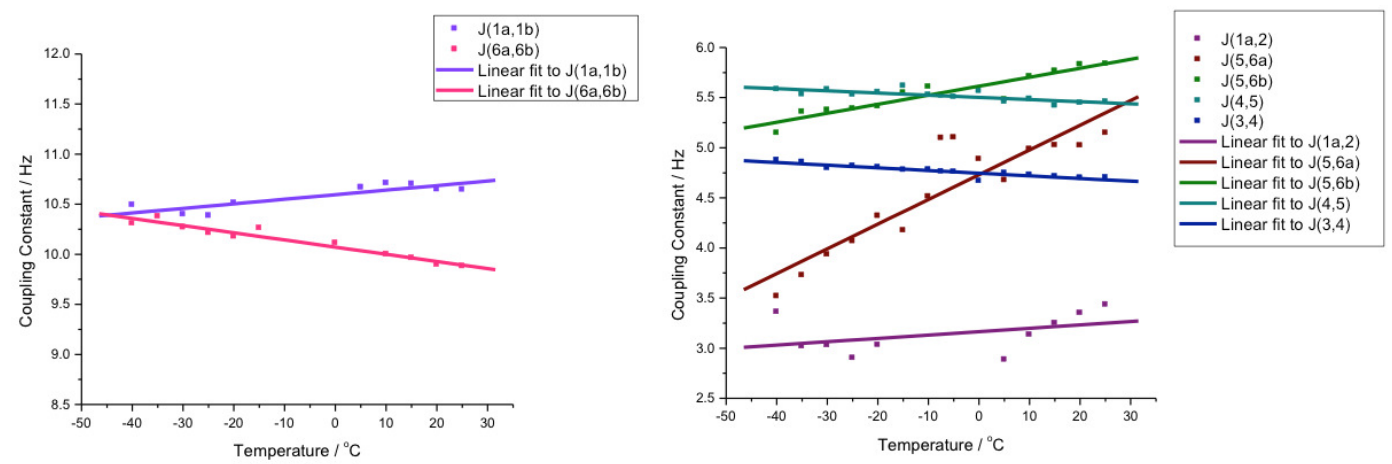

Figure 63: Coupling constant change between protons vs temperature 
$-120^{\circ} \mathrm{C}$ is calculated highest temperature for separating the peaks of the two conformation.

\subsection{Summary and Conclusions}

This study investigates the mechanism of the temperature dependence of high HTP chiral addtives. The high HTP is typically the results of the molecular structure and typically the twisting of planar aromatic rings. The temperature dependence of the HTP varies significantly with changes in the chiral additive and with changes in the nematic solvent. UV visible spectroscopy of a series of high HTP chiral additives based on an isosorbide benzoate core clearly demonstrate that the molecule has two predominant conformations and that the relative concentration of the two conformations changes substantially with temperature. The Boltzman distribution confirms that the change in the relative concentration is expected when the free energy difference between the conformations is greater than about $0.5 \mathrm{kcal} / \mathrm{mol}$ but less than $5 \mathrm{kcal} / \mathrm{mol}$. Molecular modeling supports this thesis with the isosorbide series, showing two low energy conformations of the central core resembling the boat and chair conformations familiar in cyclohexane. Molecular modeling also demonstrates a large change in the twist in the molecular structure for these two conformations and 
therefore we can expect alarge difference in their HTP. This combination of changes in the conformer distribution combined with the expected difference in the HTP of each conformer results in the large temperature dependence of the HTP. Understanding the basic mechanism of the temperature dependence of the HTP of chiral additives is the first step in designing the materials with the little or no change in HTP with temperature required for a wide variety of electro-optic applications. 


\section{CHAPTER 5}

Interaction between Chiral Dopant and Liquid Crystal

\subsection{Introduction}

From experience, one knows that once a chiral molecule dissolves into an achiral liquid crystal host, the liquid crystal generally forms a helical superstructure. However, it is not always easy to predict the handedness of the liquid crystal helix bases on the handedness of the chiral solute.

It is informative to consider the molecular factors that promote high HTP and also how these factors may impact the temperature dependence of the HTP. First, as noted in the Pieraccini [66] review high chirality is enhanced by certain molecular structures including a relatively rigid core. This promotes the stability of a twisted

chiral structure. The rigidity of the core is limited by the need for the chiral additive to be soluble in the liquid crystal host. This will require some flexibility in molecular 
structure. The balance of these requirements leads to selecting chiral additives with semi-rigid ring core structures that have two or more stable conformations. Referring back to the Boltzmann distribution and Fig 52, if these stable conformations vary in free energy by more than $.5 \mathrm{kcal} / \mathrm{mol}$ but less than $5 \mathrm{kcal} / \mathrm{mol}$ their relative distribution will change significantly over normal operating temperatures for displays and related electro-optic devices. If the HTP of these conformations is significantly different then this will result in a large temperature dependence of the HTP. In this scenario it is easy to conceive of an HTP that increase or decreases with temperature. Changing the liquid crystal host might also change the magnitude or even the sign of the temperature dependence of the HTP simply by altering the relative stability of conformers.

UV spectroscopy has the potential to shed light on how the chiral additive and the liquid crystal host interact. For example in an earlier paper Gottarelli and colleagues [67] postulate that a chiral additive induces chirality in a biaryl solvent such as cyanobiphenyl. In this case it is important to recognize that the two rings of the biphenyl are twisted about the connecting bond as a mean of reducing the steric strain found in the planar structure. The biphenyl molecule may twist either to the left or right forming an enantiomeric pair of opposite chirality. In an isotropic environment the two pairs are of equal free energy and are separated by a relatively small energy 


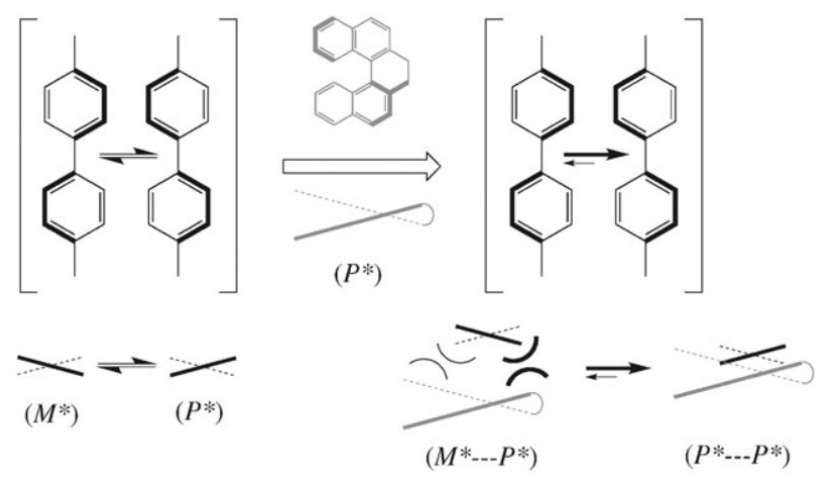

Figure 64: Two phenyl rings in the biphenyl twist conformations in either direction, and the two equal energy states switches rapidly at room temperature. Adding a chiral dopant could make one conformation lower in energy. 
barrier. Therefore, they interconvert rapidly at room temperature, shown in figure 64 As pointed out by Gottarelli, it is likely that a chiral additive will prefer nearest neighbors of one chirality, thereby breaking the energetic symmetry of the host. Simply put, a chiral additive will tend to be surrounded by solvent molecules of one chirality. This chiral induction may propagate into the nematic host. This selection should also change the UV absorption spectra of a cyanoalkylbiphenyl host.

\subsection{Experiment and Discussion}

Initial chiral dopant and liquid crystal UV Vis spectroscopy was measured in very low concentration solutions. Pure E7 and chiral dopants were dissolved in octanes, $c(E 7)=3.28 \times 10^{-5}(w t),. c(C D)=1 \times 10^{-5}-8 \times 10^{-5}(w t$.$) . The solutions are$ placed in $1 \mathrm{~cm}$ by $1 \mathrm{~cm}$ quartz cuvettes, and absorbance spectra for different temperatures are measured using a Perkin-Elmer Lambda 9 UV-Vis spectrosmeter. The background is taken with pure Octane in an identical cuvette. In Figure 65, the left figure shows the absorbance of pure E7 and pure CD6 in Octane. The E7 peak absorption is near 290nm, on the other hand CD6 peak absorption is near 260nm, and it absorbs very little light near the E7 peak. 

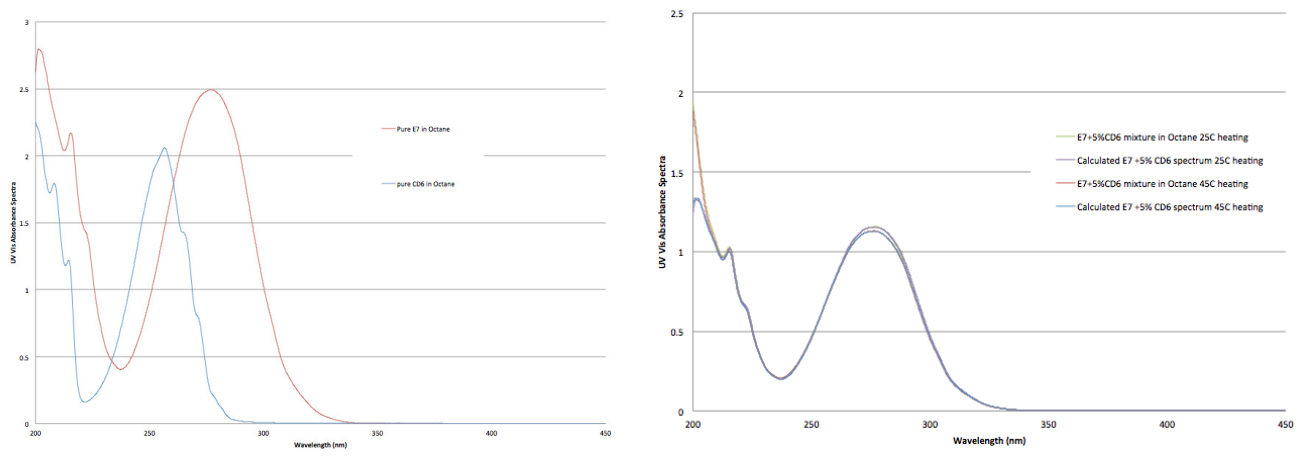

Figure 65: Left: Pure E7, Pure CD6 in Octane UV-Vis absorption spectra, $c(E 7)=$ $3.28 \times 10^{-5}(w t),. c(C D 6)=1.8 \times 10^{-5}(w t$.$) . Right: Calculated 5\% CD6 in E7 mix-$ ture spectrum matches real measurement at different temperature, which indicates no intermolecular interaction in solvent environment. 
By simply summing the E7 and CD6 spectra by weight, we get the UV-Vis spectrum for a E7 and CD6 mixture. The calculated spectrum actually matched the real measurement results, which means the in the dilute solvent environment, there is no interaction between chiral dopants and liquid crystal host. They both behave the same as they did in single compound solutions. No conformational change in the liquid crystal host is induced by adding chiral dopant into the system. We only showed the 5\%CD6 in E7 mixture case in the picture, in fact, this is proven to be true for $\mathrm{CD} 3, \mathrm{CD} 4, \mathrm{CD} 5, \mathrm{CD} 6$, with concentration ranging from $0.5 \%$ and $5 \%$, and temperature ranges between $25^{\circ} \mathrm{C}$ to $55^{\circ} \mathrm{C}$.

As an example, take E7 Octane solution, using $266 \mathrm{~g} / \mathrm{mol}$ as the E7 average molecular weight [79], and $114 \mathrm{~g} / \mathrm{mol}$ for octanes, one obtains that on the average every single E7 molecule is surrounded by 70,000 solvent molecules. Imagine putting E7 molecules in a 3D solvent lattice, every E7 molecule has approximately 40 octanes surrounding it in each direction. In such an environment, one can calculate the probability of an E7 molecule to running into another E7 molecule, and the even less probable interaction between it and a chiral dopant molecule. These results, actually, agrees with the surface chirality theory, which explains the interaction between a chiral dopant and liquid crystal host on the basis of steric interaction of nearest neighbors. This chiral interaction passes the chirality, or helicity of a chiral dopant to thee liquid crystal 
host when they are adjacent each other. Moreover, an achiral solvent, for example, octanes in this system, cannot pass the helicity between the chiral dopant and the liquid crystal.

The low concentration solution described above is an extreme case. In most applications the chiral dopant is dissolved in a neat liquid crystals solvent. The large extinction coefficients of both the chiral dopant and the liquid crystal require very thin films in order to be measured by the UV visible spectra. The mixtures are therefore applied as thin films on quartz substrates. The liquid crystal mixtures are dissolved in hexanes at concentration ranging from 0.05 and $4 \%$. The resulting solution is then spincoated onto a PI 2555 pre-coated quartz plates using a spin speed of 2000rpm. Since the PI surface are non-rubbed, the anchoring direction is randomly distributed along PI surface. Then two pieces of coated quartz plate are assembled into a cell in vacuum, as shown in Fig 66. For each cell, a background correction was done using two quartz plates. The film thickness $\mathrm{L}$ was estimated from the isotropic extinction coefficient of 5CB and E7 using the Beer Lambert equation: $A=\varepsilon c l$ [80]. The extinction coefficient of $5 \mathrm{CB}$ and E7 are $6.45 \times 10^{-3} \mathrm{~nm}^{-1}$. Film thickness were calculated as approximately $150 \mathrm{~nm}$. Since UV light source in spectrometer is partially polarized, all of the spectra are taken average of the parallel and perpendicular orientations 

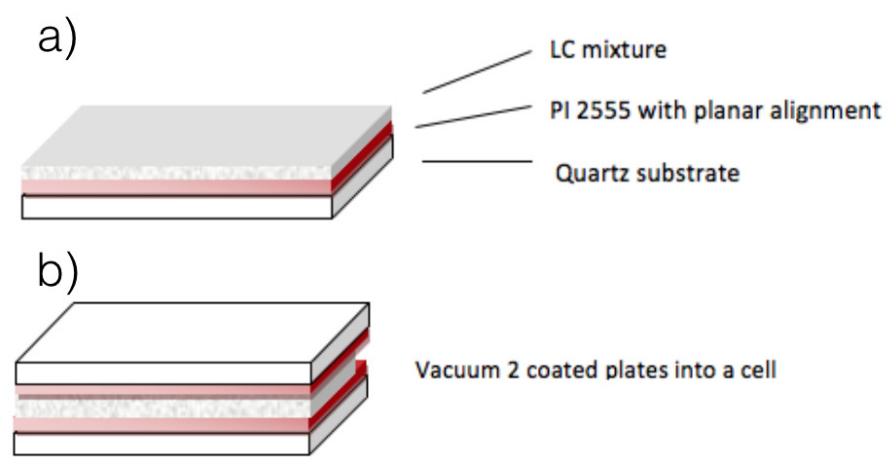

Vacuum 2 coated plates into a cell

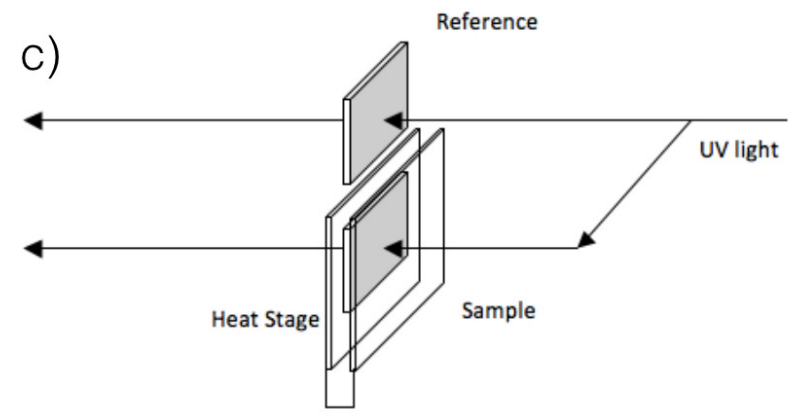

Figure 66: a) liquid crystal mixture coated on PI2555 pre-coated quartz substrate. b) vacuum assemble two identical plates into a cell. c)UV Vis spectroscopy optical path, two PI 2555 pre-coated plates as reference. 
The first mixtures measured had 3\% and $0.05 \%$ CD3 mixed in E7. E7 was used because it is a better solvent for the chiral dopant than $5 \mathrm{CB}$. For the $3 \%$ mixture, the concentration is around $1 / 27$, meaning on average, one chiral dopant exists in every $3 \times 3 \times 3$ liquid crystal lattice, $2 \mathrm{D}$ view shown in Fig 67 . Of course, the lattice structure doesn't exist in a cholesteric liquid crystals, but imagining it helps in understanding the relative position between molecules. Although the $3 \%$ concentration seems low, we can see that in a 2D lattice, on average, each liquid crystal has one nearest chiral dopant neighbor. All the liquid crystal molecules are directly influenced by chiral dopant. For $0.05 \%$ mixture, the ratio gets as low as 1 : 216 , meaning only 1 out of 9 liquid crystal molecules is adjacent to a chiral dopant molecule. The other have only secondary or even less interaction with the chiral dopant molecule.

If the chiral dopant molecule can only pass its chirality to adjacent liquid crystal molecules, then it is not clear how the helicity propagates through the bulk mixture. On the other hand, if chirality could also be passed from adjacent liquid crystal molecules to those further away in the same way, then helicity can propagate through the mixture. This indicates that the lowest energy state for adjacent liquid crystal molecules has matching twist conformations. This lowering of the free energy by matching twist conformation will impact the UV-Visible absorption spectra of the material as it passes from the isotropic to nematic phase. 
a)

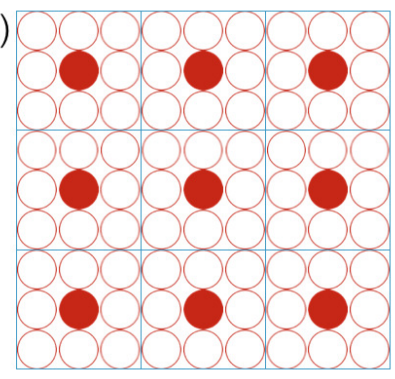

b)

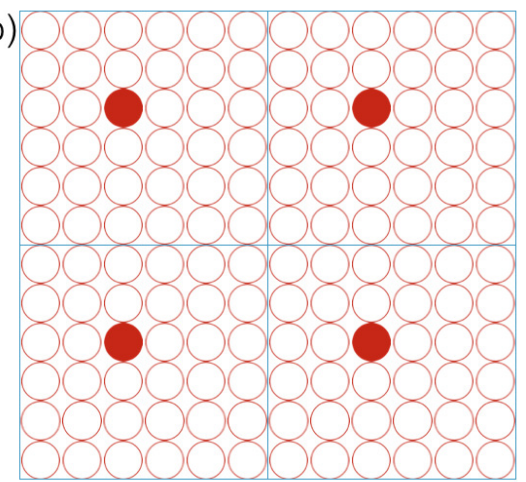

Figure 67: a) liquid crystal mixture coated on PI2555 pre-coated quartz substrate. b) vacuum assembly of two identical plates into a cell. c)UV-Vis spectroscopy optical path, two PI 2555 pre-coated plates are used as reference. 
As shown previously, the chiral dopant and liquid crystal have different absorption peaks, so we can separate the two parts by subtraction the E7 spectra from the mixture spectra.

$A_{290}($ mixture $)=A_{290}($ pureE 7$) c(E 7) r(E 7)+A_{290}($ pureCD3 $) c(C D 3) r(C D 3)+\chi_{1} A_{290}$;

$A_{260}($ mixture $)=A_{260}($ pure E7 $) c(E 7) r(E 7)+A_{260}($ pureCD3 $) c(C D 3) r(C D 3)+\chi_{2} A_{260}$;

In the equations, $\mathrm{A}$ is $\mathrm{UV}$ Vis absorbance at each wavelength. And $\mathrm{c}(\mathrm{E} 7)$ and $\mathrm{c}(\mathrm{CD} 3)$ represent to concentration of $\mathrm{E} 7$ and $\mathrm{CD} 3$, and $\mathrm{r}(\mathrm{E} 7)$ and $\mathrm{r}(\mathrm{CD} 3)$ represent the thickness ratio of E7 and CD3 films compared to the mixture film, respectively. $\chi$ is an indicator, which reflects chiral dopant and liquid crystal interaction. As we know in solution spectrum, the CD3 has no absorbance at $290 \mathrm{~nm}, \chi_{1}$ term equals 0 .

Scenario 1, assuming only the surface interaction happens in mixtures, for mixtures below $3 \%$ (the critical concentration that, in average, every host molecule could 
interact with chiral dopant directly), $\chi_{2}$ value should be proportional to c(CD), meaning the interaction is limited by the number of the chiral dopant molecules, and adding more liquid crystal in the mixture doesn?t induce more interaction. Scenario 2, long-distance interaction between liquid crystal and chiral dopant. In this case the interaction increase when fitting more and more liquid crystal molecules between chiral dopant molecules, so $\chi_{2}$ should be a constant.

By fitting the spectra, we can get value of $\chi_{2}$ for each mixture, such as shown in Figure 68 . When plotting $\chi_{2}$ vs the concentration of chiral dopant, we get Figure 69, which shows the actual measurement results fit neither of the scenarios. In fact, when the concentration is lower than the critical concentration, although some of the liquid crystals are further away from the chiral dopant, they are still get influenced by the chiral dopant. However, this influence is not a long distance interaction, since van der Waals force decrease dramatically at large distance. It is more like the helicity of the chiral dopant gets "passed" to the nearest neighbor liquid crystal, then the interaction between liquid crystal molecules, and helicity gets "passed" to the next liquid crystal molecule. Helicity gets "passed" one-by-one, and finally gets through the liquid crystal bulk. Unfortunately, the helicity of one liquid crystal molecule cannot be copied exactly when passed to another, otherwise, it fits the long-distance scenario again. It gets reduced a little bit every time it is passed. So we see $\chi_{2}$ gets 

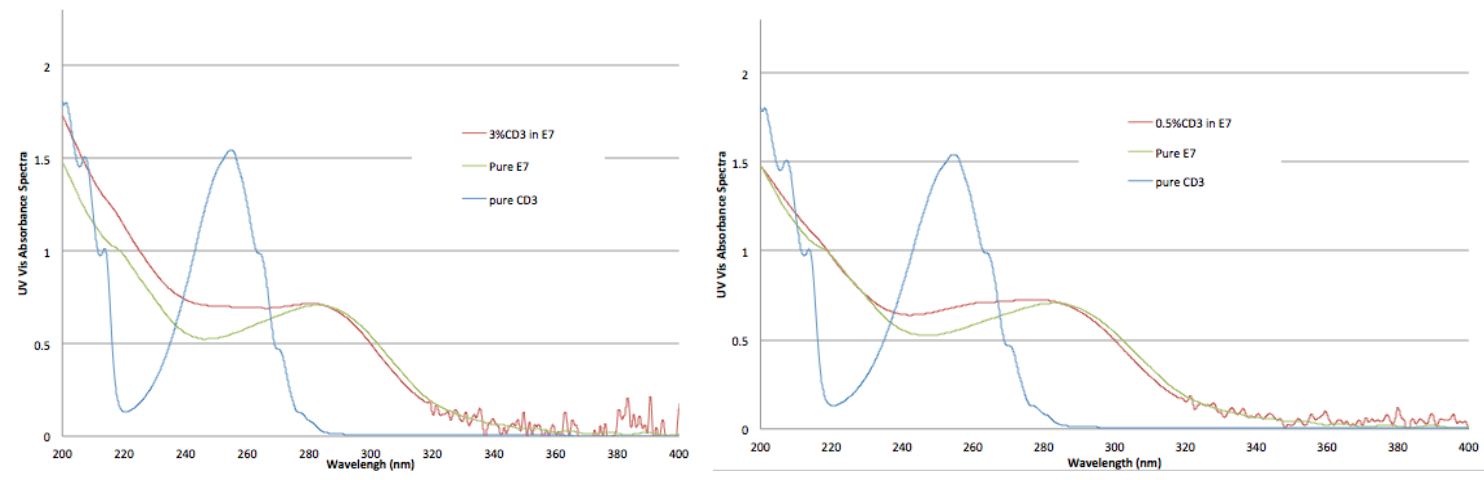

Figure 68: a) thin film UV Vis spectra of 3\% of CD3 dissolved in E7 b)thin film UV Vis spectra of $3 \%$ of CD3 dissolved in E7 


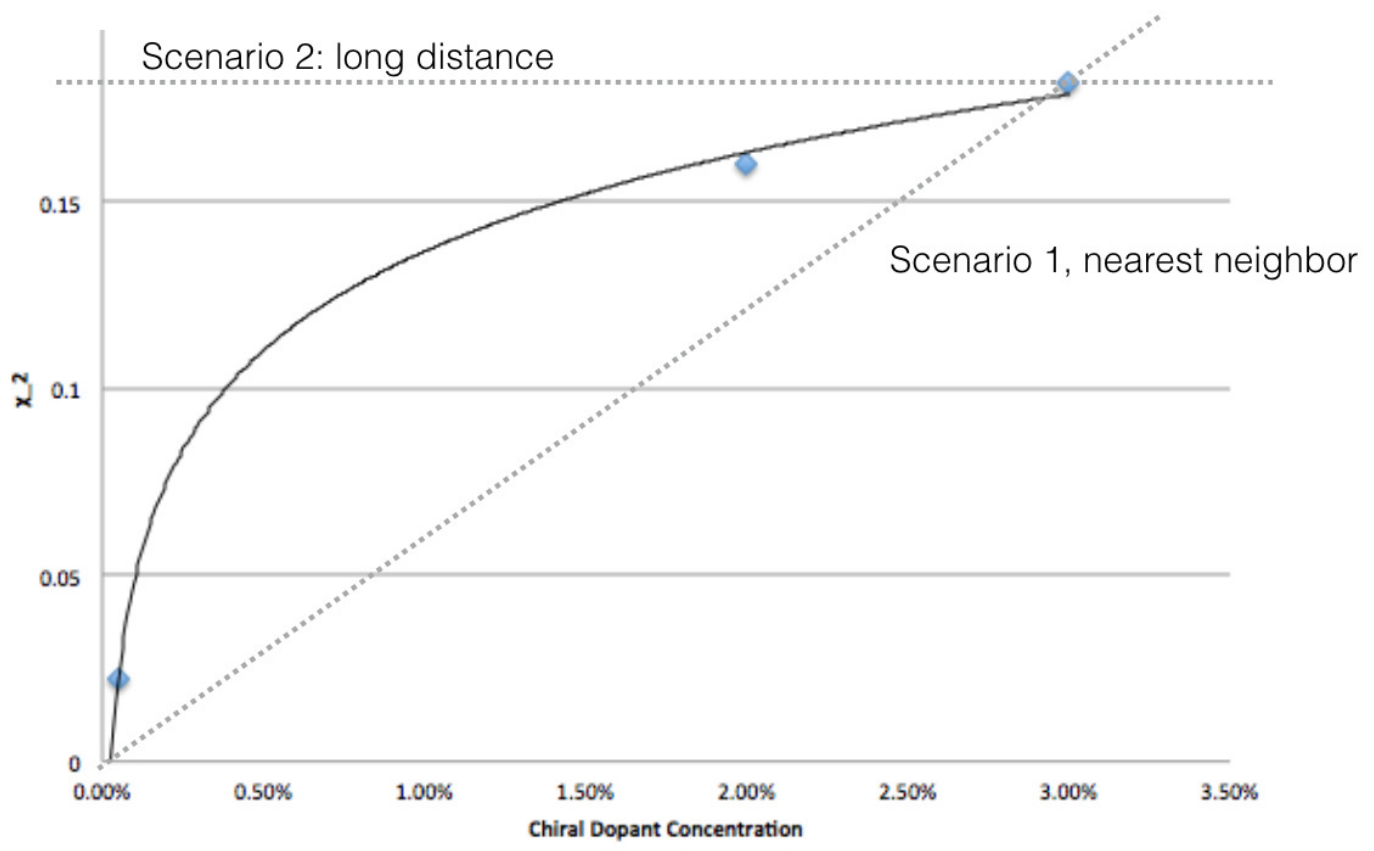

Figure 69: $\chi_{2}$ vs concentration of chiral dopant 
smaller when the chiral dopant concentration gets lower.

Imagine we can removed one single chiral dopant from the mixture, what will happen? At the beginning, although all of the molecules can have their biphenyl conformations switching back and forth, since all of the surrounding molecules are of the same helicity, the original helicity is preferred more than the other. There's a high chance that one liquid crystal molecule switches helicity will switch back to original state. The whole system stays the same helicity as is, until several liquid crystal molecules in the bulk "accidentally" switches to the other helicity together, then this new helicity could propagate though the bulk, however, without the chiral dopant, the helicity influence is weaker, and liquid crystal molecules can switch back and forth more easily. At the end, all the liquid crystal molecules ends up at a random helicity, and the system become achiral.

Another interesting phenomenon we observed is the UV Vis spectra dependence of pure liquid crystal films and chiral doped liquid crystal films. As shown in Figures 70 and 71 , we found pure liquid crystal films have a much larger temperature dependence than chiral doped liquid crystal films. This could caused by the order parameter reduction at higher temperature. As shown in earlier publication [80] the parallel absorbance is higher than perpendicular absorbance. Since all the UV Vis 

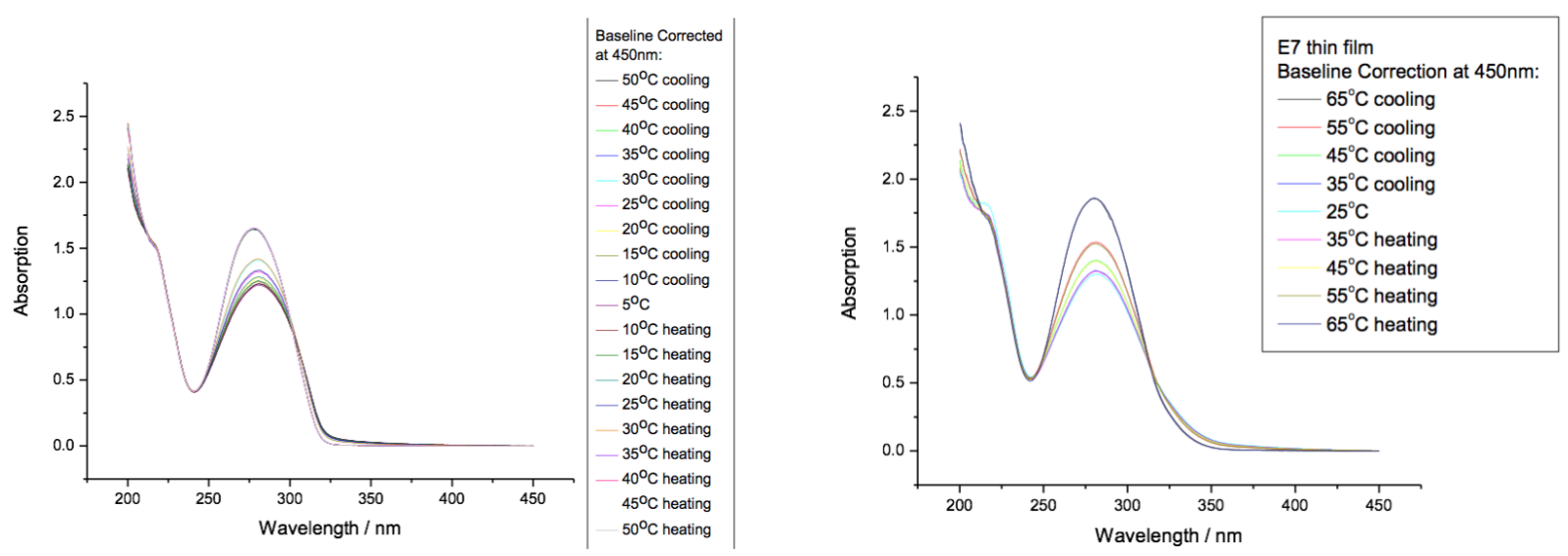

Figure 70: Left: 5CB thin film cell UV-Vis absorption spectra at different temperature. Right: E7 thin film cell UV-Vis absorption spectra at different temperture 

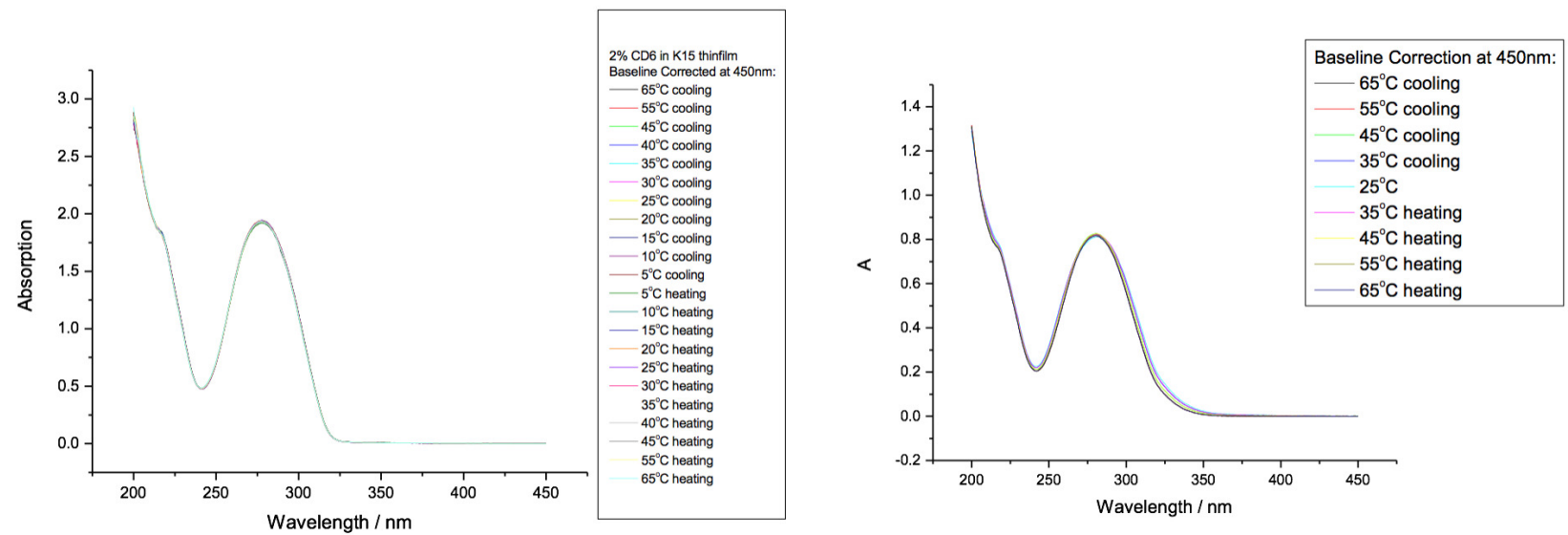

Figure 71: Left: 5CB with 2\% CD6 thin film cell UV-Vis absorption spectra at different temperature. Right: E7 with 3\%CD6 thin film cell UV-Vis absorption spectra at different temperture 
spectroscopy is partially polarized, and thin film absorbance is measured in a perpendicular direction, when temperature gets closer to $T_{N I}$, the probability that a molecule ends up with parallel to incident polarization increases, which leads to a higher absorption peak. In the cholesteric case, the molecular already form a helical structure and the temperature change impact to average molecular direction is small, so the UV-Vis spectra show small dependence with temperature.

\subsection{Conclusion}

In this Chapter, measurements of liquid crystal-dopant interactions are presented. The goal is to understand the interaction between the liquid crystal and chiral dopant. UV-Vis spectra in solvent shows chirality cannot be passed by achiral molecules. The thin film spectra results extended the surface chirality theory and demonstrated how is chirality propagate in liquid crystal bulk. 


\section{CHAPTER 6}

\section{Conclusion}

Chirality, as an interesting topic that has been drawing attention from many fields. It is not only of academic interest, it also influence sour daily life. When chirality applies its magic in the liquid crystal world, it brings tremendous amount of opportunities.

In this dissertation, we tried to under how the molecular chirality transfers into helicity in bulk liquid crystal samples; or effectively how the micro scale chirality transfers into macro scale chirality. Molecular dynamic modeling helped us understand the conformational distribution change of chiral dopants when the temperature varies. At a certain temperature, the chiral dopant chirality is easier to pass to the environmental liquid crystal molecules than at another temperature. This feature could impact the temperature dependence of helical twisting power.

The increasing demand for chiral liquid crystal applications drew more research on this topic. More study is necessary for better understanding of the interaction between liquid crystals and chiral dopants, and finally, this knowledge can be used in molecular structure design. We already know that, without conformational change, helical twisting power decreases when temperature increases due to an entropy effect. 
so, If we can design a system, which has a conformer distribution has opposite impact as the entropy impact, ideally, with the same amount of impact on opposite direction, we could get an "ideal chiral dopant", which has no temperature dependence. Of course, this ideal chiral dopant should only work for a specific liquid crystal, as we known, the interaction between liquid crystal and chiral dopant plays a big role, too. 


\section{BIBLIOGRAPHY}

[1] L. D. Barron, "Chemistry: compliments from Lord Kelvin." Nature, vol. 446, no. 7135, pp. 505-6, mar 2007. [Online]. Available: http: //www.nature.com/nature/journal/v446/n7135/full/446505a.html $\{\backslash \#\}$ close

[2] I. Kant, "Kant's Prolegomena: and Metaphysical foundations of natural science," pp. P32-P33, 1883. [Online]. Available: https://books.google.com/books?id= OCJLAAAAMAAJ $\{\backslash \&\} \mathrm{pg}=\mathrm{PA} 32\{\backslash \&\} \mathrm{lpg}=\mathrm{PA} 32\{\backslash \&\} \mathrm{dq}=$ what + can + more + resemble + my + hand + or + my + ear $\{\backslash \&\}$ source $=$ bl $\{\backslash \&\}$ ots $=\mathrm{B} 7 \mathrm{yxDS} 89 \mathrm{Tu}\{\backslash \&$ \} sig $=$ YADrB1ktb $\{\backslash$ - $H$ HQAYPkqo7ZXnsWj7g $\{\backslash \&\} \mathrm{hl}=\operatorname{en}\{\backslash \&\}$ sa $=\mathrm{X}\{\backslash \&$

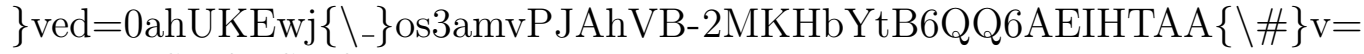
twopage $\{\backslash \&\} \mathrm{q}\{\backslash \&\} \mathrm{f}=$ false

[3] August Ferdinand Möbius, Der Barycentrische Calcul: Ein Neues Hülfsmittel zur Analytischen Behandlung der Geometrie. (German Edition), 2011. [Online]. Available: http://www.amazon.com/ Barycentrische-Calcul-Hlfsmittel-Analytischen-Behandlung/dp/1247742474/ ref $=\operatorname{sr}\left\{\backslash \backslash_{-}\right\}\{\backslash\} 1$ ? s=books $\{\backslash \&\}$ ie $=\mathrm{UTF} 8\{\backslash \&\} \mathrm{qid}=1451444491\{\backslash \&\} \mathrm{sr}=1-1\{\backslash \&$ \} keywords=Der+barycentrische+Calcul

[4] E. A. Abbott, Flatland:A Romance of Many Dimensions, 1884. [Online]. Available: http://www.eldritchpress.org/eaa/FL.HTM

[5] s. a. T. magazine. Project Ozma was a pioneering SETI experiment started in 1960 by Cornell University astronomer Frank Drake, at the National Radio Astronomy Observatory at Green Bank, West Virginia. The object of the experiment was to search for signs of life in distant p, "The object of the experiment was to search for signs of life in distant planetary systems through interstellar radio waves."

[6] "Four Out of Five Recent Presidents Are Southpaws - ABC News." [Online]. Available: http://abcnews.go.com/Politics/Vote2008/story?id=4326568\{\\& \}page $=1$

[7] Sima Qian, Records of the Grand Historian.

[8] L. Pasteur, "Mémoire sur la relation qui peut exister entre la forme cristalline et la composition chimique, et sur la cause de la polarisation rotatoire" (Memoir on 
the relationship which can exist between crystalline form and chemical composition, and on the cause o," Comptes rendus de l'Académie des sciences (Paris), vol. vol. 26, pp. pp. 535-538., 1848.

[9] Annales de chimie et de physique, Volume 24, 1848. [Online]. Available: https://books.google.com/books?id=gJ45AAAAcAAJ $\{\backslash \&\}$ pgis $=1$

[10] H. D. Flack, "Louis Pasteurs discovery of molecular chirality and spontaneous resolution in 1848, together with a complete review of his crystallographic and chemical work," Acta Crystallographica Section A: Foundations of Crystallography, vol. 65, no. 5, pp. 371-389, 2009.

[11] R. B. Grossman, "Van't Hoff, Le Bel, and the development of stereochemistry: A reassessment," Journal of Chemical Education, vol. 66, no. 1, p. 30, jan 1989. [Online]. Available: http://dx.doi.org/10.1021/ed066p30

[12] T. J. Leitereg, D. G. Guadagni, J. Harris, T. R. Mon, and R. Teranishi, "Chemical and sensory data supporting the difference between the odors of the enantiomeric carvones," Journal of Agricultural and Food Chemistry, vol. 19, no. 4, pp. 785-787, jul 1971. [Online]. Available: http: //dx.doi.org/10.1021/jf60176a035

[13] M. Laska, A. Liesen, and P. Teubner, "Enantioselectivity of odor perception in squirrel monkeys and humans." The American journal of physiology, vol. 277, no. 4 Pt 2, pp. R1098-R1103, 1999. [Online]. Available: http://ajpregu.physiology.org/content/277/4/R1098.full-text.pdf+html

[14] "Organic Chemistry, 6th Edition: K. Peter C. Vollhardt, Neil E. Schore: 9781429204941: Amazon.com: Books." [Online]. Available: http://www. amazon.com/Organic-Chemistry-Edition-Peter-Vollhardt/dp/142920494X

[15] J. Simonsen, The terpenes. Cambridge: at the University Press, 1947.

[16] F. Reinitzer, "Beiträge zur Kenntniss des Cholesterins," Monatshefte für Chemie - Chemical Monthly, vol. 9, no. 1, pp. 421-441, dec 1888. [Online]. Available: http://link.springer.com/10.1007/BF01516710

[17] G. Solladié and R. G. Zimmermann, "Liquid Crystals: A Tool for Studies on Chirality," Angewandte Chemie International Edition in English, vol. 23, no. 5, pp. 348-362, may 1984. [Online]. Available: http: //doi.wiley.com/10.1002/anie.198403481

[18] H.-S. Kitzerow and C. Bahr, Eds., Chirality in Liquid Crystals, ser. Partially Ordered Systems. New York: Springer-Verlag, 2001. [Online]. Available: http://link.springer.com/10.1007/b97374 
[19] P. G. de Gennes and J. Prost, The Physics of Liquid Crystals. Clarendon Press, 1995. [Online]. Available: https://books.google.com/books?id= $0 \mathrm{Nw}-\mathrm{dzWz} 5 \mathrm{agC}\{\backslash \&\}$ pgis $=1$

[20] Handbook of Liquid Crystals, Low Molecular Weight Liquid Crystals I: Calamitic Liquid Crystals. Wiley, 1998. [Online]. Available: https: //books.google.com/books?id=apjHsgEACAAJ $\{\backslash \&\}$ pgis $=1$

[21] S. Chandrasekhar, Liquid Crystals. Cambridge: Cambridge University Press, 1992. [Online]. Available: /ebook.jsf?bid=CBO9780511622496

[22] P. Collings, "Introduction to liquid crystals chemistry and physics," London ;BBristol, PA :, 1997.

[23] I. Dierking, Textures of Liquid Crystals. John Wiley \& Sons, 2003. [Online]. Available: https://books.google.com/books?id=p-0xdzDRB2kC $\{\backslash \&\}$ pgis $=1$

[24] A. Jakli and A. Saupe, One- and Two-Dimensional Fluids: Properties of Smectic, Lamellar and Columnar Liquid Crystals. CRC Press, 2006. [Online]. Available: https://books.google.com/books?id=rGfLBQAAQBAJ $\{\backslash \&\}$ pgis=1

[25] R. Mezzenga, P. Schurtenberger, A. Burbidge, and M. Michel, "Understanding foods as soft materials." Nature materials, vol. 4, no. 10, pp. 729-40, oct 2005. [Online]. Available: http://dx.doi.org/10.1038/nmat1496

[26] H. Kikuchi, Liquid Crystalline Functional Assemblies and Their Supramolecular Structures, ser. Structure and Bonding, T. Kato, Ed. Berlin, Heidelberg: Springer Berlin Heidelberg, 2008, vol. 128. [Online]. Available: http: //www.springerlink.com/index/10.1007/978-3-540-77867-7

[27] T. Seideman, "The liquid-crystalline blue phases," Reports on Progress in Physics, vol. 53, no. 6, pp. 659-706, 1999.

[28] H. J. Coles and M. N. Pivnenko, "Liquid crystal 'blue phases' with a wide temperature range." Nature, vol. 436, no. 7053, pp. 997-1000, aug 2005. [Online]. Available: http://dx.doi.org/10.1038/nature03932

[29] A. Yoshizawa, M. Sato, and J. Rokunohe, "A blue phase observed for a novel chiral compound possessing molecular biaxiality," Journal of Materials Chemistry, vol. 15, no. 32, p. 3285, aug 2005. [Online]. Available: http://pubs.rsc.org/en/content/articlehtml/2005/jm/b506167a

[30] H. Kikuchi, M. Yokota, Y. Hisakado, H. Yang, and T. Kajiyama, "Polymerstabilized liquid crystal blue phases." Nature materials, vol. 1, no. 1, pp. 64-8, sep 2002. [Online]. Available: http://dx.doi.org/10.1038/nmat712 
[31] T. Carlsson, F. M. Leslie, and N. A. Clark, "Macroscopic theory for the flow behavior of smectic- C and smectic- C * liquid crystals," Physical Review E, vol. 51, no. 5, pp. 4509-4525, may 1995. [Online]. Available: http://link.aps.org/doi/10.1103/PhysRevE.51.4509

[32] P. Das, S. Basu, R. Sinha, and U. Das, "Dipole-induced chiral smecticC phase in a eutectic mixture of cholesterol esters," Chemical Physics Letters, vol. 410, no. 4-6, pp. 417-422, jul 2005. [Online]. Available: http://www.sciencedirect.com/science/article/pii/S0009261405008080

[33] R. Breier, S. Herminghaus, and M. G. Mazza, "Spontaneous chiral symmetry breaking in model bacterial suspensions," vol. 1, pp. 2-5, 2014. [Online]. Available: http://arxiv.org/abs/1406.4423

[34] C. J. Eckhardt, N. M. Peachey, D. R. Swanson, J. M. Takacs, M. A. Khan, X. Gong, J.-H. Kim, J. Wang, and R. A. Uphaus, "Separation of chiral phases in monolayer crystals of racemic amphiphiles," Nature, vol. 362, no. 6421, pp. 614-616, apr 1993. [Online]. Available: http://dx.doi.org/10.1038/362614a0

[35] G. Pickett, M. Gross, and H. Okuyama, "Spontaneous chirality in simple systems," Physical review letters, vol. 85, no. 17, pp. 3652-5, oct 2000. [Online]. Available: http://www.ncbi.nlm.nih.gov/pubmed/11030973

[36] B. Freelon, M. Ramazanoglu, P. J. Chung, R. N. Page, Y.-T. Lo, P. Valdivia, C. W. Garland, and R. J. Birgeneau, "Smectic- ¡math display="inline" $i$ ¡mrowi mi $_{i} \mathrm{~A}_{i} / \mathrm{mi}_{i} i /$ mrow $_{i} i /$ math $_{i}$ and smectic- math display $="$ inline" $i$ ¡mrowi $i_{\text {mi }} \mathrm{C}_{\mathrm{i}} / \mathrm{mi}_{i} \mathrm{i} / \mathrm{mrow}_{i} \mathrm{i} / \mathrm{math}_{i}$ phases and phase transitions in $i m a t h$

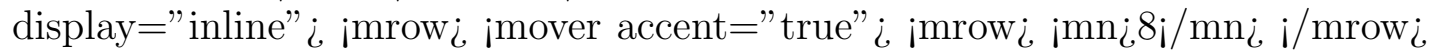
¡mrow:," Physical Review E, vol. 84, no. 3, p. 031705, 2011. [Online]. Available: http://link.aps.org/doi/10.1103/PhysRevE.84.031705

[37] F. C. Frank, "I. Liquid crystals. On the theory of liquid crystals," Discussions of the Faraday Society, vol. 25, p. 19, jan 1958. [Online]. Available: http://pubs.rsc.org/en/content/articlehtml/1958/df/df9582500019

[38] U. Wilson, Mark R (University of Durham and U. Earl, David J (University of Durham, "Calculating the helical twisting power of chiral dopants," Jounal of Materials Chemistry, vol. 11, pp. 2672-2677, 2001.

[39] Peter Palffy-Muhoray, "Peter Palffy-Muhoray Liquid Crystal Optics Class Notes."

[40] W. Cao, "Fluorescence and Lasing in Liquid Crystalline Photonic Bandgap Materials," Ph.D. dissertation, 2005. 
[41] D.-k. Yang and S.-T. Wu, Fundamentals of Liquid Crystal Devices, 2006.

[42] C. Gerritsma, W. Goossens, and A. Niessen, "The helical twist in a cholesteric Grandjean-Cano pattern," Physics Letters A, vol. 34, no. 7, pp. 354-355, apr 1971. [Online]. Available: http://www.sciencedirect.com/science/article/ pii/0375960171909108

[43] T.-T. Tang, H.-Y. Wu, C.-J. Lin, and R.-P. Pan, "Optical method for measuring the azimuthal anchoring strength of liquid crystals using pitch values determined in imperfect samples," Journal of Applied Physics, vol. 102, no. 6, p. 063108, 2007. [Online]. Available: http://scitation.aip.org/content/aip/journal/ jap/102/6/10.1063/1.2781320

[44] F. J. Kahn, "No Title," Phys. Rev. Lett., no. 24, p. 209, 1970.

[45] W. D. St. John, W. J. Fritz, Z. J. Lu, and D.-K. Yang, "Bragg reflection from cholesteric liquid crystals," Physical Review E, vol. 51, no. 2, pp. 1191-1198, feb 1995. [Online]. Available: http://link.aps.org/doi/10.1103/PhysRevE.51.1191

[46] P. D. Brimicombe and E. P. Raynes, "Symmetric H state lifetime in splayed nematic liquid crystal devices," Applied Physics Letters, vol. 89, no. 3, p. 031121, jul 2006. [Online]. Available: http://adsabs.harvard.edu/abs/ 2006ApPhL..89c1121B

[47] J. W. Goodby, I. M. Saez, S. J. Cowling, V. Görtz, M. Draper, A. W. Hall, S. Sia, G. Cosquer, S.-E. Lee, and E. P. Raynes, "Transmission and amplification of information and properties in nanostructured liquid crystals." Angewandte Chemie (International ed. in English), vol. 47, no. 15, pp. 2754-87, jan 2008. [Online]. Available: http://www.ncbi.nlm.nih.gov/pubmed/18311737

[48] J. W. Raynes, Peter; Cowling, Stephen J.; Goodby, P. Raynes, J. Cowling, and J. W. Goodby, "Investigations of optical activity of natural products and chiral pharmaceuticals using liquid crystal technologies - Research Database, The University of York," pp. 88-92, 2009.

[49] S.-W. Suh, K. Joseph, G. Cohen, J. S. Patel, S.-D. Lee, S.-W. Suh, K. Joseph, G. Cohen, J. S. Patel, and S.-D. Lee, "Precise determination of the cholesteric pitch of a chiral liquid crystal in a circularly aligned configuration," Applied Physics Letters, vol. 70, no. 19, p. 2547, may 1997. [Online]. Available: http://scitation.aip.org/content/aip/journal/apl/70/19/10.1063/1.118916

[50] M. V. Vasnetsov, D. S. Kasyanyuk, I. P. Terenetskaya, P. S. Kapinos, and V. V. Slyusar, "Disclination Line in $\theta$-Cell as an Indicator of Liquid Crystal Chirality," Molecular Crystals and Liquid Crystals, vol. 575, no. 1, pp. 57-63, 
jun 2013. [Online]. Available: http://www.tandfonline.com/doi/abs/10.1080/ 15421406.2013.766945?journalCode=gmcl20\{\\#\}.VraSATbnC-I

[51] D. Kasyanyuk, K. Slyusarenko, J. West, M. Vasnetsov, and Y. Reznikov, "Formation of liquid-crystal cholesteric pitch in the centimeter range." Physical review. E, Statistical, nonlinear, and soft matter physics, vol. 89, no. 2, p. 022503, feb 2014. [Online]. Available: http://journals.aps.org/pre/abstract/10. 1103/PhysRevE.89.022503

[52] M. Stalder and M. Schadt, "Linearly polarized light with axial symmetry generated by liquid-crystal polarization converters," Optics Letters, vol. 21, no. 23, p. 1948, dec 1996. [Online]. Available: http://www.osapublishing.org/ viewmedia.cfm?uri $=$ ol-21-23-1948 $\{\backslash \&\}$ seq $=0\{\backslash \&\}$ html $=$ true

[53] R. Memmer; H.-G. Kuball and A.Schönhofer, "Computer simulation of chiral liquid crystal phases III. A cholesteric phase formed by chiral GayBerne atropisomers," pp. 89, 1633-1649, 1996. [Online]. Available: https: //www.chemie.uni-kl.de/fachrichtungen/pc/memmer/abst.html\{\\#\}publ006

[54] D. Podolskyy, O. Banji, and P. Rudquist, "Simple method for accurate measurements of the cholesteric pitch using a stripewedge GrandjeanCano cell," Liquid Crystals, vol. 35, no. 7, pp. 789-791, jul 2008. [Online]. Available: http://www.tandfonline.com/doi/abs/10.1080/02678290802175756

[55] J. W. Goodby, "Optical activity and ferroelectricity in liquid crystals." Science (New York, N.Y.), vol. 231, no. 4736, pp. 350-5, jan 1986. [Online]. Available: http://science.sciencemag.org/content/231/4736/350.abstract

[56] K.-U. Jeong, S. Jin, J. J. Ge, B. S. Knapp, M. J. Graham, J. Ruan, M. Guo, H. Xiong, F. W. Harris, and S. Z. D. Cheng, "Phase Structures and Self-assembled Helical Suprastructures via Hydrogen Bonding in a Series of Achiral 4-Biphenyl Carboxylic Acid Compounds," Chemistry of Materials, vol. 17, no. 11, pp. 2852-2865, may 2005. [Online]. Available: http://dx.doi.org/10.1021/cm050338y

[57] K.-U. Jeong, B. S. Knapp, J. J. Ge, S. Jin, M. J. Graham, F. W. Harris, and S. Z. D. Cheng, "Origin of Self-Assembled Helical Supramolecular Structures in Achiral C6 Biphenyl Carboxylic Acid Compounds," Chemistry of Materials, vol. 18, no. 3, pp. 680-690, feb 2006. [Online]. Available: http://dx.doi.org/10.1021/cm0518832

[58] K.-U. Jeong, D.-K. Yang, M. Graham, Y. Tu, S.-W. Kuo, B. Knapp, F. Harris, and S. Cheng, "Construction of Chiral Propeller Architectures from Achiral Molecules," Advanced Materials, vol. 18, no. 24, pp. 3229-3232, dec 2006. [Online]. Available: http://doi.wiley.com/10.1002/adma.200601338 
[59] J. Wang, C. Y. Li, S. Jin, X. Weng, R. M. Van Horn, M. J. Graham, W.-B. Zhang, K.-U. Jeong, F. W. Harris, B. Lotz, and S. Z. D. Cheng, "Helical Crystal Assemblies in Nonracemic Chiral Liquid Crystalline Polymers: Where Chemistry and Physics Meet ," Industrial 85 Engineering Chemistry Research, vol. 49, no. 23, pp. 11936-11947, dec 2010. [Online]. Available: http://dx.doi.org/10.1021/ie100248r

[60] K. S. P. J. D. Kemp, J. D. Kemp, and K. S. Pitzer, "The Entropy of Ethane and the Third Law of Thermodynamics. Hindered Rotation of Methyl Groups," Journal of the American Chemical Society, vol. 59, no. 2, pp. 276279, feb 1937. [Online]. Available: http://dx.doi.org/10.1021/ja01281a014http: //pubs.acs.org/doi/pdf/10.1021/ja01281a014

[61] O. Bastiansen and S. Samdal, "Structure and barrier of internal rotation of biphenyl derivatives in the gaseous state," Journal of Molecular Structure, vol. 128, no. 1-3, pp. 115-125, apr 1985. [Online]. Available: http://www.sciencedirect.com/science/article/pii/0022286085850444

[62] A. J. Cruz-Cabeza, J. W. Liebeschuetz, and F. H. Allen, "Systematic conformational bias in small-molecule crystal structures is rare and explicable," CrystEngComm, vol. 14, no. 20, p. 6797, sep 2012. [Online]. Available: http://pubs.rsc.org/en/content/articlehtml/2012/ce/c2ce25585e

[63] O. Bastiansen and S. Samdel, "Structure and barrier of internal rotation of biphenyl derivatives in the gaseous state. Part 4. Barrier of internal rotation in biphenyl, perdeuterated biphenyl and seven non-orthosubstituted halogen derivatives," pp. 115-125, 1985. [Online]. Available: http://ac.els-cdn.com/0022286085850444/1-s2.0-0022286085850444-main.pdf? $\{\backslash$ - $\}$ tid=edb5f8e4-c7bb-11e5-b293-00000aacb361 $\{\backslash \&\}$ acdnat $=1454204746\{\backslash$ - $\}$ a121a2e9caee699442a46f8cfb7695ba

[64] "Conformations of Biphenyls - Chemwiki." [Online]. Available: http://chemwiki.ucdavis.edu/Organic $\{\backslash\}$ Chemistry/Chirality/Stereoisomers/ Chirality $\left\{\backslash \backslash_{-}\right\}$and $\left\{\backslash_{-}\right\}$Symmetry/Enantiomorphism/Conformational $\{\backslash$ - $\}$ Enantiomorphism/Conformations $\left\{\backslash \backslash_{-}\right\}$of $\left\{\backslash_{-}\right\}$Biphenyls

[65] P. E. M. S. U. William Reusch, "Virtual Textbook of Organic Chemistry."

[66] S. Pieraccini, A. Ferrarini, and G. P. Spada, "Chiral doping of nematic phases and its application to the determination of absolute configuration." Chirality, vol. 20, no. 5, pp. 749-59, may 2008. [Online]. Available: http://www.ncbi.nlm.nih.gov/pubmed/17924420 
[67] A. Ferrarini and P. Nordio, "Simple Molecular Model for Induced Cholesteric Phases," Physical Review E, vol. 53, no. 1, pp. 681-688, 1996.

[68] H. Sachse, "No Title," Chem. Ber, vol. 23, p. 1363, 1890.

[69] —_, "No Title," Z. Physik. Chem, vol. 10, p. 203, 1892.

[70] —_, "No Title," Z. Physik. Chem, vol. 11, pp. 183-219, 1893.

[71] I. Pieraccini, Silvia (University of Bologna, I. Masiero, Stefano (University of Bologna, I. Ferrarini, Alberta (University of Padova, and I. Piero Spada, Gian (University of Bologna, "Chirality transfer across length-scales in nematic liquid crystals: fundamentals and applications," Chemical Society reviews, vol. 40, no. 1, pp. 258-71, jan 2011. [Online]. Available: http://www.ncbi.nlm.nih.gov/pubmed/20938496

[72] D. H. Ron Naaman, David N. Beratan, Electronic and Magnetic Properties of Chiral Molecules and Supramolecular Architectures. Springer Science \& Business Media, 2011. [Online]. Available: https://books.google.com/books? $\mathrm{hl}=\mathrm{en}\{\backslash \&\} \mathrm{lr}=\{\backslash \&\} \mathrm{id}=1 \mathrm{fL}-\mathrm{uNIDHpsC}\{\backslash \&\}$ pgis $=1$

[73] W. P. Mose and P. M. Scopes, "Optical rotatory dispersion and circular dichroism. Part LXXI. Low temperature circular dichroism of some tertiary carboxylic acids," Journal of the Chemical Society C: Organic, p. 1572, jan 1971. [Online]. Available: http://pubs.rsc.org/en/content/articlehtml/1971/j3/ j39710001572

[74] G. G. A. o. S. Chilaya, F. T. U. B. Oestreicher, and G. G. A. o. S. Scherowsky, "Statistical Model For the Temperature Dependence of Pitch in Induced Cholesteric Liquid Crystals," Molecular Materials, vol. 9, pp. 261-269, 1998.

[75] A. J. Slaney, I. Nishiyama, P. Styring, and J. W. Goodby, "Twist inversion in a cholesteric material containing a single chiral centre," Journal of Materials Chemistry, vol. 2, no. 8, p. 805, jan 1992. [Online]. Available: http://pubs.rsc.org/en/content/articlehtml/1992/jm/jm9920200805

[76] "Chiral dopants," apr 2001. [Online]. Available: http://www.google.com/ patents/US6217792

[77] "Chiral diole compounds, liquid crystal compositions and polymer networks derived therefrom," oct 2007. [Online]. Available: http://www.google.com/ patents/WO2007120459A2?cl=en 
[78] J. Simpson, Organic Structure Determination Using 2-D NMR Spectroscopy, 2008.

[79] E. Naegele, "Quantification of Compounds in the E7 Liquid Crystal Mixture by Supercritical Fluid Chromatography with UV Detection," pp. 1-8.

[80] J. L. West, G. R. Magyar, J. R. Kelly, S. Kobayashi, Y. Iimura, and N. Yoshida, "Dichroic Ultraviolet-Absorption of Thin Liquid- Crystal Films," vol. 67, pp. 155-157, 1995.

[81] "isosbestic point," IUPAC Compendium of Chemical Terminology (2nd Edition), no. 66, p. 1129, 1997. [Online]. Available: http://old.iupac.org/goldbook/ I03310.pdf

[82] R. Sanjeev, V. Jagannadham, and R. V. Vrath, "Demonstration Of Isosbestic Point : A Novel Experiment," vol. 14, no. 2, pp. 1-6, 2012.

[83] R. Sanjeev, V. Jagannadham, and R. Vrath, "Implications of a novel interpretation of the isosbestic - Technische Informationsbibliothek (TIB)," pp. 133-134, 2012. [Online]. Available: https://www.tib.eu/de/suchen/id/BLSE $\{\ \%$ \}3ARN320768480/Implications-of-a-novel-interpretation-of-the-isosbestic/

[84] S. Gallot and O. Thomas, "Fast and easy interpretation of a set of absorption spectra: theory and qualitative applications for UV examination of waters and wastewaters," Fresenius' Journal of Analytical Chemistry, vol. 346, no. 10-11, pp. 976-983, 1993. [Online]. Available: http: //link.springer.com/10.1007/BF00322762

[85] P. Meakin, J. P. Jesson, and C. A. Tolman, "Nature of chlorotris(triphenylphosphine)rhodium in solution and its reaction with hydrogen," Journal of the American Chemical Society, vol. 94, no. 9, pp. 3240-3242, may 1972. [Online]. Available: http://dx.doi.org/10.1021/ja00764a061

[86] M. D. Cohen and E. Fischer, "588. Isosbestic points," Journal of the Chemical Society (Resumed), p. 3044, jan 1962. [Online]. Available: http://pubs.rsc.org/en/content/articlehtml/1962/jr/jr9620003044

[87] M. F. Pouët, E. Baures, S. Vaillant, and O. Thomas, "Hidden isosbestic point(s) in ultraviolet spectra," pp. 486-490, 2004. 
Appendix: Isosbestic Point

The term of isosbestic point is usually employed with reference to a set of absorption spectra when spectra are plotted on the same chart for a set of solutions in which the sum of the concentrations of two principal absorbing components is constant. The curves of absorbance against wavelength (or frequency) for such a set of mixtures often all intersect at one or more points, called isosbestic points. [81] The existence of an isosbestic point means a quality and quantity conservation in the global composition of samples with a given relation between the concentration of absorbing compounds or mixtures.

One common use of isosbestic points in chemistry is to monitor chemical reactions. When the two absorbing components concerned are a reactant and a product [82] [83], or the two absorbing components are in equilibrium in a solution. One typical example for the second use is the acid-base indicator equilibrium. [84]

$$
A \rightleftharpoons B+H_{a q}^{+}
$$




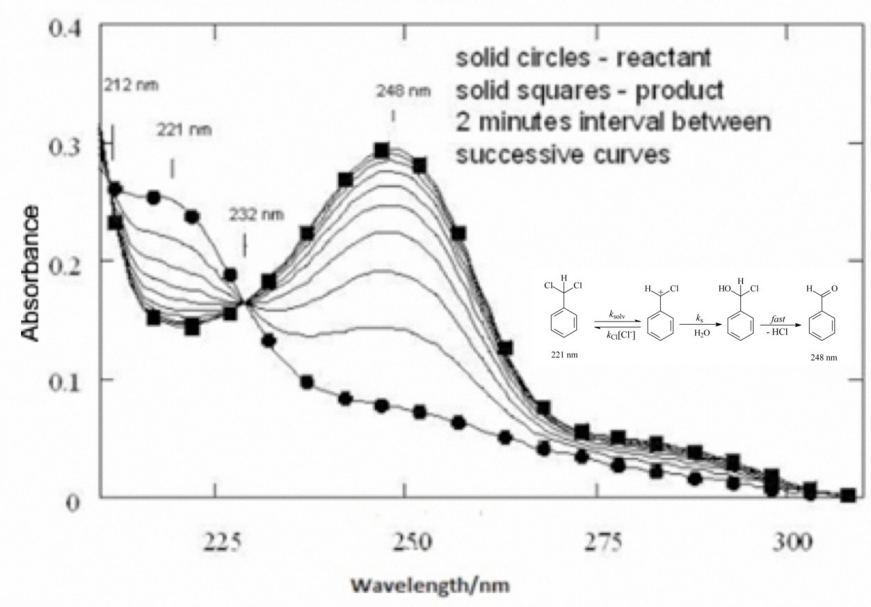

Figure 72: Time dependent UV-VIS spectrum of (dichloromethyl)benzene in water at $25^{\circ} \mathrm{C}$ 
Although some specific cases are reported, the existence of isosbestic point is often considered proof of the presence of two absorbing species in the analyzed solution [84] [85]. There is no general agreement on the conditions required for presence of isosbestic point; however, some general trends can be found [86] [87]:

(1) the system is closed or results need to be corrected to correspond to a fixed over all concentration; (2) the spectra of the limiting states of system intersect (3) the changes in the concentrations of the various components are linearly related, i.e., only one degree of freedom exist.

When considering the possibility of more than two absorbing components are existing in systems, the isosbestic point is less likely to occur. The conditions to form an isosbestic point are then more complicated. Therefore, the existence of isosbestic points can be linked to a two-component system. When an isosbestic point exists, it means both of the below conditions are achieved:

(1) There is a fixed linear relation between the concentrations of the two components or pseudo-components;

(2) the spectra for the two components intersect inside the chose wavelength interval. 